\title{
Readiness of the ATLAS Tile Calorimeter for LHC collisions
}

\author{
The ATLAS Collaboration ${ }^{\star, \star \star}$
}

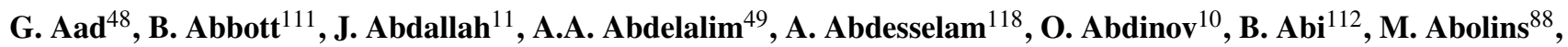
H. Abramowicz ${ }^{153}$, H. Abreu ${ }^{115}$, B.S. Acharya ${ }^{164 a, 164 b}$, D.L. Adams ${ }^{24}$, T.N. Addy ${ }^{56}$, J. Adelman ${ }^{175}$, C. Adorisio ${ }^{36 a, 36 b}$, P. Adragna ${ }^{75}$, T. Adye $^{129}$, S. Aefsky ${ }^{22}$, J.A. Aguilar-Saavedra ${ }^{124 b, a}$, M. Aharrouche ${ }^{81}$, S.P. Ahlen ${ }^{21}$, F. Ahles ${ }^{48}$, A. Ahmad ${ }^{148}$, M. Ahsan ${ }^{40}$, G. Aielli ${ }^{133 a, 133 b}$, T. Akdogan ${ }^{18 a}$, T.P.A. Åkesson ${ }^{79}$, G. Akimoto $^{155}$, A.V. Akimov ${ }^{94}$, A. Aktas $^{48}$, M.S. Alam ${ }^{1}$, M.A. Alam ${ }^{76}$, S. Albrand ${ }^{55}$, M. Aleksa ${ }^{29}$, I.N. Aleksandrov ${ }^{65}$, C. Alexa ${ }^{25 a}$, G. Alexander ${ }^{153}$, G. Alexandre ${ }^{49}$, T. Alexopoulos ${ }^{9}$, M. Alhroob ${ }^{20}$, M. Aliev ${ }^{15}$, G. Alimonti ${ }^{89}$, J. Alison ${ }^{120}$, M. Aliyev ${ }^{10}$, P.P. Allport ${ }^{73}$, S.E. Allwood-Spiers ${ }^{53}$, J. Almond ${ }^{82}$, A. Aloisio ${ }^{102 a, 102 b}$, R. Alon ${ }^{171}$, A. Alonso ${ }^{79}$, M.G. Alviggi ${ }^{102 a, 102 b}$, K. Amako ${ }^{66}$, C. Amelung ${ }^{22}$, A. Amorim ${ }^{124 a, b}$, G. Amorós ${ }^{167}$, N. Amram ${ }^{153}$, C. Anastopoulos ${ }^{139}$, T. Andeen ${ }^{29}$, C.F. Anders ${ }^{48}$, K.J. Anderson ${ }^{30}$, A. Andreazza ${ }^{89 a, 89 b}$, V. Andrei ${ }^{58 a}$, X.S. Anduaga ${ }^{70}$, A. Angerami ${ }^{34}$, F. Anghinolfi ${ }^{29}$, N. Anjos ${ }^{124 a}$, A. Annovi ${ }^{47}$, A. Antonaki ${ }^{8}$, M. Antonelli ${ }^{47}$, S. Antonelli ${ }^{19 a, 19 b}$, J. Antos ${ }^{14 b}$, B. Antunovic ${ }^{41}$, F. Anulli ${ }^{132 a}$, S. Aoun ${ }^{83}$, G. Arabidze $^{8}$, I. Aracena ${ }^{143}$, Y. Arai ${ }^{66}$, A.T.H. Arce ${ }^{44}$, J.P. Archambault ${ }^{28}$, S. Arfaoui ${ }^{29, c}$, J.-F. Arguin ${ }^{14}$, T. Argyropoulos ${ }^{9}$, M. Arik ${ }^{18 a}$, A.J. Armbruster ${ }^{87}$, O. Arnaez ${ }^{4}$, C. Arnault $^{115}$, A. Artamonov ${ }^{95}$, D. Arutinov ${ }^{20}$, M. Asai ${ }^{143}$, S. Asai ${ }^{155}$, R. Asfandiyarov ${ }^{172}$, S. Ask ${ }^{82}$, B. Åsman ${ }^{146 a, 146 b}$, D. Asner ${ }^{28}$, L. Asquith ${ }^{77}$, K. Assamagan ${ }^{24}$, A. Astvatsatourov ${ }^{52}$, G. Atoian ${ }^{175}$, B. Auerbach ${ }^{175}$, K. Augsten ${ }^{127}$, M. Aurousseau ${ }^{4}$, N. Austin ${ }^{73}$, G. Avolio ${ }^{163}$, R. Avramidou ${ }^{9}$, C. Ay ${ }^{54}$, G. Azuelos ${ }^{93, \mathrm{~d}}$, Y. Azuma ${ }^{155}$, M.A. Baak ${ }^{29}$, A.M. Bach ${ }^{14}$, H. Bachacou ${ }^{136}$, K. Bachas ${ }^{29}$, M. Backes $^{49}$, E. Badescu ${ }^{25 a}$, P. Bagnaia ${ }^{132 a, 132 b}$, Y. Bai ${ }^{32 a}$, T. Bain ${ }^{158}$, J.T. Baines ${ }^{129}$, O.K. Baker ${ }^{175}$, M.D. Baker ${ }^{24}$, S. Baker ${ }^{77}$, F. Baltasar Dos Santos Pedrosa ${ }^{29}$, E. Banas ${ }^{38}$, P. Banerjee ${ }^{93}$, S. Banerjee ${ }^{169}$, D. Banfi ${ }^{89,89 b}$,

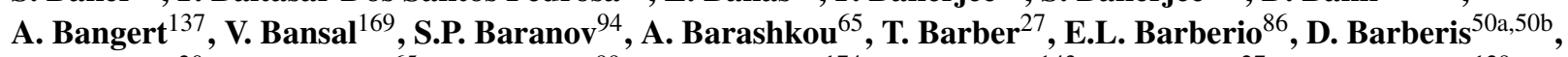
M. Barbero ${ }^{20}$, D.Y. Bardin ${ }^{65}$, T. Barillari ${ }^{99}$, M. Barisonzi ${ }^{174}$, T. Barklow ${ }^{143}$, N. Barlow ${ }^{27}$, B.M. Barnett ${ }^{129}$, R.M. Barnett ${ }^{14}$, A. Baroncelli ${ }^{134 a}$, A.J. Barr ${ }^{118}$, F. Barreiro ${ }^{80}$, J. Barreiro Guimarães da Costa $^{57}$, P. Barrillon ${ }^{115}$, R. Bartoldus ${ }^{143}$, D. Bartsch ${ }^{20}$, R.L. Bates ${ }^{53}$, L. Batkova ${ }^{144 a}$, J.R. Batley ${ }^{27}$, A. Battaglia ${ }^{16}$, M. Battistin ${ }^{29}$, F. Bauer ${ }^{136}$, H.S. Bawa ${ }^{143}$, M. Bazalova ${ }^{125}$, B. Beare ${ }^{158}$, T. Beau ${ }^{78}$, P.H. Beauchemin ${ }^{118}$, R. Beccherle ${ }^{50 a}$, P. Bechtle ${ }^{41}$,

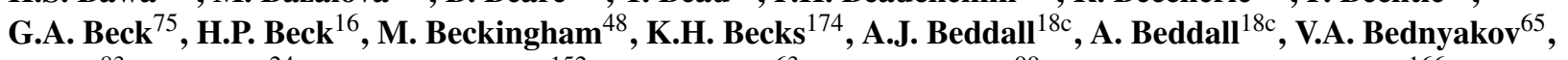
C. Bee ${ }^{83}$, M. Begel ${ }^{24}$, S. Behar Harpaz ${ }^{152}$, P.K. Behera ${ }^{63}$, M. Beimforde ${ }^{99}$, C. Belanger-Champagne ${ }^{166}$, P.J. Bell ${ }^{49}$, W.H. Bell ${ }^{49}$, G. Bella ${ }^{153}$, L. Bellagamba ${ }^{19 a}$, F. Bellina ${ }^{29}$, M. Bellomo ${ }^{119 a}$, A. Belloni ${ }^{57}$, K. Belotskiy ${ }^{96}$, O. Beltramello $^{29}$, S. Ben Ami ${ }^{152}$, O. Benary ${ }^{153}$, D. Benchekroun ${ }^{135 a}$, M. Bendel ${ }^{81}$, B.H. Benedict ${ }^{163}$, N. Benekos ${ }^{165}$, Y. Benhammou ${ }^{153}$, D.P. Benjamin ${ }^{44}$, M. Benoit ${ }^{115}$, J.R. Bensinger ${ }^{22}$, K. Benslama ${ }^{130}$, S. Bentvelsen ${ }^{105}$, M. Beretta ${ }^{47}$, D. Berge ${ }^{29}$, E. Bergeaas Kuutmann ${ }^{41}$, N. Berger ${ }^{4}$, F. Berghaus ${ }^{169}$, E. Berglund ${ }^{49}$, J. Beringer ${ }^{14}$, P. Bernat ${ }^{115}$, R. Bernhard ${ }^{48}$, C. Bernius ${ }^{77}$, T. Berry ${ }^{76}$, A. Bertin ${ }^{19 a, 19 b}$, M.I. Besana ${ }^{89 a, 89 b}$, N. Besson ${ }^{136}$, S. Bethke ${ }^{99}$, R.M. Bianchi ${ }^{48}$, M. Bianco ${ }^{72 a, 72 b}$, O. Biebel ${ }^{98}$, J. Biesiada ${ }^{14}$, M. Biglietti ${ }^{132 a, 132 b}$, H. Bilokon $^{47}$, M. Bindi ${ }^{19 a, 19 b}$,

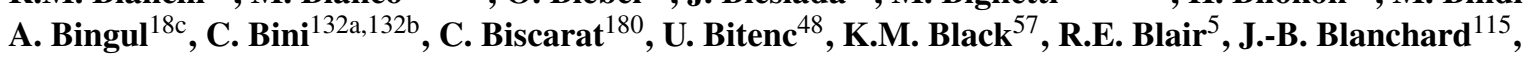
G. Blanchot $^{29}$, C. Blocker ${ }^{22}$, A. Blonde ${ }^{49}$, W. Blum ${ }^{81}$, U. Blumenschein ${ }^{54}$, G.J. Bobbink ${ }^{105}$, A. Bocci ${ }^{44}$, M. Boehler ${ }^{41}$, J. Boek ${ }^{174}$, N. Boelaert ${ }^{79}$, S. Böser ${ }^{77}$, J.A. Bogaerts ${ }^{29}$, A. Bogouch ${ }^{90,{ }^{*} \text {, C. Bohm }{ }^{146 a} \text {, J. Bohm }}{ }^{125}$, V. Boisvert ${ }^{76}$, T. Bold ${ }^{163, \mathrm{e}}$, V. Boldea ${ }^{25 a}$, V.G. Bondarenko ${ }^{96}$, M. Bondioli ${ }^{163}$, M. Boonekamp ${ }^{136}$, S. Bordoni $^{78}$, C. Borer ${ }^{16}$, A. Borisov ${ }^{128}$, G. Borissov ${ }^{71}$, I. Borjanovic ${ }^{12 a}$, S. Borroni ${ }^{132 a, 132 b}$, K. Bos ${ }^{105}$, D. Boscherini ${ }^{19 a}$, M. Bosman ${ }^{11}$, H. Boterenbrood ${ }^{105}$, J. Bouchami ${ }^{93}$, J. Boudreau ${ }^{123}$, E.V. Bouhova-Thacker ${ }^{71}$, C. Boulahouache ${ }^{123}$, C. Bourdarios ${ }^{115}$, A. Boveia ${ }^{30}$, J. Boyd ${ }^{29}$, I.R. Boyko ${ }^{65}$, I. Bozovic-Jelisavcic ${ }^{12 b}$, J. Bracinik ${ }^{17}$, A. Braem ${ }^{29}$,

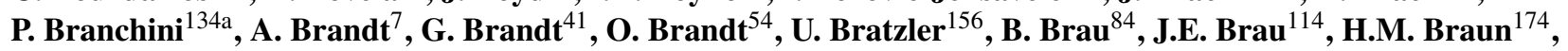
B. Brelier ${ }^{158}$, J. Bremer ${ }^{29}$, R. Brenner ${ }^{166}$, S. Bressler ${ }^{152}$, D. Britton ${ }^{53}$, F.M. Brochu ${ }^{27}$, I. Brock ${ }^{20}$, R. Brock ${ }^{88}$, E. Brodet ${ }^{153}$, C. Bromberg ${ }^{88}$, G. Brooijmans ${ }^{34}$, W.K. Brooks ${ }^{31 b}$, G. Brown ${ }^{82}$, P.A. Bruckman de Renstrom ${ }^{38}$, D. Bruncko $^{144 b}$, R. Bruneliere ${ }^{48}$, S. Brunet ${ }^{41}$, A. Bruni ${ }^{19 a}$, G. Bruni ${ }^{19 a}$, M. Bruschi $^{19 a}$, F. Bucci ${ }^{49}$, J. Buchanan ${ }^{118}$, P. Buchholz ${ }^{141}$, A.G. Buckley ${ }^{45}$, I.A. Budagov ${ }^{65}$, B. Budick ${ }^{108}$, V. Büscher ${ }^{81}$, L. Bugge ${ }^{117}$, O. Bulekov ${ }^{96}$, M. Bunse ${ }^{42}$,

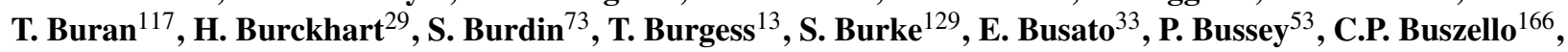
F. Butin ${ }^{29}$, B. Butler ${ }^{143}$, J.M. Butler ${ }^{21}$, C.M. Buttar ${ }^{53}$, J.M. Butterworth ${ }^{77}$, T. Byatt ${ }^{77}$, J. Caballero ${ }^{24}$, S. Cabrera 
Urbán $^{167}$, D. Caforio ${ }^{19 a, 19 b}$, O. Cakir ${ }^{3 a}$, P. Calafiura ${ }^{14}$, G. Calderini ${ }^{78}$, P. Calfayan ${ }^{98}$, R. Calkins ${ }^{106}$, L.P. Caloba ${ }^{23 a}$, D. Calvet $^{33}$, P. Camarri ${ }^{133 a, 133 b}$, D. Cameron ${ }^{117}$, S. Campana ${ }^{29}$, M. Campanelli ${ }^{77}$, V. Canale ${ }^{102 a, 102 b}$, F. Canelli ${ }^{30}$, A. Canepa ${ }^{159 a}$, J. Cantero ${ }^{80}$, L. Capasso ${ }^{102 a, 102 b}$, M.D.M. Capeans Garrido $^{29}$, I. Caprini2 ${ }^{25 a}$, M. Caprini ${ }^{25 a}$, M. Capua ${ }^{36 a, 36 b}$, R. Caputo ${ }^{148}$, C. Caramarcu ${ }^{25 a}$, R. Cardarelli $^{133 a}$, T. Carli $^{29}$, G. Carlino $^{102 a}$, L. $_{\text {Carminati }}{ }^{89 a, 89 b}$,

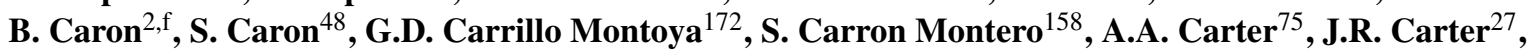
J. Carvalho ${ }^{124 a, g}$, D. Casadei ${ }^{108}$, M.P. Casado ${ }^{11}$, M. Cascella ${ }^{122 a, 122 b}$, A.M. Castaneda Hernandez ${ }^{172}$,

E. Castaneda-Miranda ${ }^{172}$, V. Castillo Gimenez ${ }^{167}$, N.F. Castro ${ }^{124 b, a}$, G. Cataldi ${ }^{72 a}$, A. Catinaccio ${ }^{29}$, J.R. Catmore $^{71}$,

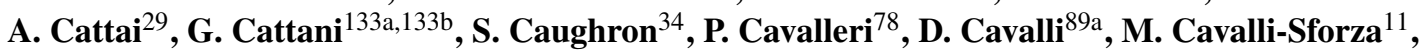
V. Cavasinni ${ }^{122 a, 122 b}$, F. Ceradini ${ }^{134 a, 134 b}$, A.S. Cerqueira ${ }^{23 a}$, A. Cerri ${ }^{29}$, L. Cerrito $^{75}$, F. Cerutti $^{47}$, S.A. Cetin $^{18 b}$, A. Chafaq ${ }^{135 a}$, D. Chakraborty ${ }^{106}$, K. Chan $^{2}$, J.D. Chapman ${ }^{27}$, J.W. Chapman ${ }^{87}$, E. Chareyre ${ }^{78}$, D.G. Charlton ${ }^{17}$, V. Chavda $^{82}$, S. Cheatham ${ }^{71}$, S. Chekanov ${ }^{5}$, S.V. Chekulaev ${ }^{159 a}$, G.A. Chelkov ${ }^{65}$, H. Chen $^{24}$, S. Chen $^{32 c}$, X. Chen $^{172}$, A. Cheplakov ${ }^{65}$, V.F. Chepurnov ${ }^{65}$, R. Cherkaoui El Moursli ${ }^{135 d}$, V. Tcherniatine ${ }^{24}$, D. Chesneanu ${ }^{25 a}$, E. Cheu ${ }^{6}$, S.L. Cheung ${ }^{158}$, L. Chevalier ${ }^{136}$, F. Chevallier ${ }^{136}$, G. Chiefari ${ }^{102 a, 102 b}$, L. Chikovani ${ }^{51}$, J.T. Childers ${ }^{58 a}$, A. Chilingarov ${ }^{71}$, G. Chiodini ${ }^{72 a}$, V. Chizhov ${ }^{65}$, G. Choudalakis $^{30}$, S. Chouridou ${ }^{137}$, I.A. Christidi ${ }^{77}$, A. Christov $^{48}$, D. Chromek-Burckhart ${ }^{29}$, M.L. Chu ${ }^{151}$, J. Chudoba ${ }^{125}$, G. Ciapetti ${ }^{132 a, 132 b}$, A.K. Ciftci $^{3 a}$, R. Ciftci ${ }^{3 a}$, D. Cinca ${ }^{33}$, V. Cindro ${ }^{74}$, M.D. Ciobotaru ${ }^{163}$, C. Ciocca ${ }^{19 a, 19 b}$, A. Ciocio ${ }^{14}$, M. Cirilli ${ }^{87, h}$, A. Clark $^{49}$, P.J. Clark ${ }^{45}$, W. Cleland ${ }^{123}$, J.C. Clemens ${ }^{83}$, B. Clement ${ }^{55}$, C. Clement ${ }^{146 a, 146 b}$, Y. Coadou ${ }^{83}$, M. Cobal ${ }^{164 a, 164 c}$, A. Coccaro ${ }^{50 a, 50 b}$, J. Cochran ${ }^{64}$, J. Coggeshall ${ }^{165}$, E. Cogneras ${ }^{180}$, A.P. Colijn ${ }^{105}$, C. Collard ${ }^{115}$, N.J. Collins ${ }^{17}$, C. Collins-Tooth ${ }^{53}$, J. Collot $^{55}$, G. Colon $^{84}$, P. Conde Muiño ${ }^{124 a}$, E. Coniavitis ${ }^{166}$, M.C. Conidi ${ }^{11}$, M. Consonni ${ }^{104}$, S. Constantinescu $^{25 a}$, C. Conta ${ }^{119 a, 119 b}$, F. Conventi ${ }^{102 a, i}$, M. Cooke ${ }^{34}$, B.D. Cooper $^{75}$, A.M. Cooper-Sarkar ${ }^{118}$, N.J. Cooper-Smith ${ }^{76}$, K. Copic $^{34}$, T. Cornelissen ${ }^{50 a, 50 b}$, M. Corradi ${ }^{19 a}$, F. Corriveau ${ }^{85, j}$, A. Corso-Radu ${ }^{163}$, A. Cortes-Gonzalez ${ }^{165}$, G. Cortiana ${ }^{99}$, G. Costa ${ }^{89 a}$, M.J. Costa ${ }^{167}$, D. Costanzo ${ }^{139}$, T. Costin ${ }^{30}$, D. Côtée ${ }^{29}$, R. Coura Torres ${ }^{23 a}$, L. Courneyea ${ }^{169}$, G. Cowan ${ }^{76}$, C. Cowden ${ }^{27}$, B.E. Cox ${ }^{82}$, K. Cranmer ${ }^{108}$, J. Cranshaw ${ }^{5}$, M. Cristinziani ${ }^{20}$, G. Crosetti ${ }^{36 a, 36 b}$, R. Crupi ${ }^{72 a, 72 b}$, S. Crépé-Renaudinn ${ }^{55}$, C. Cuenca Almenar ${ }^{175}$, T. Cuhadar Donszelmannn ${ }^{139}$, M. Curatolo ${ }^{47}$, C.J. Curtis ${ }^{17}$, P. Cwetanski ${ }^{61}$, Z. Czyczula ${ }^{175}$, S. D'Auria ${ }^{53}$, M. D'Onofrio ${ }^{73}$, A. D’Orazio ${ }^{99}$, C. Da Via ${ }^{82}$, W. Dabrowski ${ }^{37}$, T. Dai ${ }^{87}$, C. Dallapiccola ${ }^{84}$, S.J. Dallison ${ }^{129, *}$, C.H. Daly ${ }^{138}$, M. Dam ${ }^{35}$, H.O. Danielsson ${ }^{29}$, D. Dannheim ${ }^{99}$, V. Dao ${ }^{49}$, G. Darbo ${ }^{50 a}$, G.L. Darlea ${ }^{25 b}$, W. Davey ${ }^{86}$, T. Davidek ${ }^{126}$, N. Davidson ${ }^{86}$, R. Davidson ${ }^{71}$, M. Davies ${ }^{93}$, A.R. Davison ${ }^{77}$, I. Dawson ${ }^{139}$, R.K. Daya ${ }^{39}$, K. De ${ }^{7}$, R. de Asmundis ${ }^{102 a}$, S. De Castro ${ }^{19 a}$,19b , P.E. De Castro Faria Salgado ${ }^{24}$, S. De Cecco ${ }^{78}$, J. de Graat ${ }^{98}$, N. De Groot ${ }^{104}$, P. de Jong ${ }^{105}$, L. De Mora ${ }^{71}$, M. De Oliveira Branco ${ }^{29}$, D. De Pedis ${ }^{132 a}$, A. De Salvo ${ }^{132 a}$, U. De Sanctis ${ }^{164 a, 164 c}$, A. De Santo ${ }^{149}$, J.B. De Vivie De Regie ${ }^{115}$, S. Dean ${ }^{77}$, D.V. Dedovich ${ }^{65}$, J. Degenhardt ${ }^{120}$, M. Dehchar ${ }^{118}$, C. Del Papa ${ }^{164 a, 164 c}$, J. Del Peso ${ }^{80}$, T. Del Prete ${ }^{122 a, 122 b}$, A. Dell'Acqua ${ }^{29}$, L. Dell'Asta $^{89,89 b}$, M. Della Pietra $^{102 a, k}$, D. della Volpe ${ }^{102 a, 102 b}$, M. Delmastro ${ }^{29}$, P.A. Delsart ${ }^{55}$, C. Deluca ${ }^{148}$, S. Demers ${ }^{175}$, M. Demichev ${ }^{65}$, B. Demirkoz ${ }^{11}$, J. Deng ${ }^{163}$, W. Deng ${ }^{24}$, S.P. Denisov ${ }^{128}$, J.E. Derkaoui ${ }^{135 c}$, F. Derue ${ }^{78}$, P. Dervan $^{73}$, K. Desch $^{20}$, P.O. Deviveiros ${ }^{158}$, A. Dewhurst ${ }^{129}$, B. DeWilde ${ }^{148}$, S. Dhaliwal ${ }^{158}$, R. Dhullipudi ${ }^{24,1}$, A. Di Ciaccio ${ }^{133 a, 133 b}$, L. Di Ciaccio ${ }^{4}$, A. Di Girolamo ${ }^{29}$, B. Di Girolamo ${ }^{29}$, S. Di Luise ${ }^{134 a, 134 b}$, A. Di Mattia ${ }^{88}$, R. Di Nardo ${ }^{133 a, 133 b}$, A. Di Simone ${ }^{133 a, 133 b}$, R. Di Sipio ${ }^{19 a, 19 b}$, M.A. Diaz ${ }^{31 a}$, F. Diblen ${ }^{18 c}$, E.B. Diehl ${ }^{87}$, J. Dietrich ${ }^{48}$, T.A. Dietzsch ${ }^{58 a}$, S. Diglio ${ }^{115}$, K. Dindar Yagci ${ }^{39}$, J. Dingfelder ${ }^{48}$, C. Dionisi ${ }^{132 a, 132 b}$, P. Dita ${ }^{25 a}$, S. Dita ${ }^{25 a}$, F. Dittus ${ }^{29}$, F. Djama ${ }^{83}$, R. Djilkibaev ${ }^{108}$, T. Djobava ${ }^{51}$, M.A.B. do Vale ${ }^{23 a}$, A. Do Valle Wemans ${ }^{124 a}$, T.K.O. Doan $^{4}$, D. Dobos ${ }^{29}$, E. Dobson ${ }^{29}$, M. Dobson ${ }^{163}$, C. Doglioni ${ }^{118}$, T. Doherty ${ }^{53}$, J. Dolejsi ${ }^{126}$, I. Dolenc ${ }^{74}$, Z. Dolezal ${ }^{126}$, B.A. Dolgoshein ${ }^{96}$, T. Dohmae ${ }^{155}$, M. Donega ${ }^{120}$, J. Donini ${ }^{55}$, J. Dopke $^{174}$, A. Doria ${ }^{102 a}$, A. Dos Anjos ${ }^{172}$, A. Dotti ${ }^{122 a, 122 b}$, M.T. Dova ${ }^{70}$, A. Doxiadis ${ }^{105}$, A.T. Doyle ${ }^{53}$, Z. Drasal ${ }^{126}$, M. Dris ${ }^{9}$, J. Dubbert ${ }^{99}$, E. Duchovni ${ }^{171}$, G. Duckeck ${ }^{98}$, A. Dudarev ${ }^{29}$, F. Dudziak ${ }^{115}$, M. Dührssen ${ }^{29}$, L. Duflot ${ }^{115}$, M.-A. Dufour ${ }^{85}$, M. Dunford ${ }^{30}$, H. Duran Yildiz ${ }^{3 b}$, R. Duxfield ${ }^{139}$, M. Dwuznik ${ }^{37}$, M. Düren ${ }^{52}$, W.L. Ebenstein ${ }^{44}$, J. Ebke ${ }^{98}$, S. Eckweiler ${ }^{81}$, K. Edmonds ${ }^{81}$, C.A. Edwards ${ }^{76}$, K. Egorov ${ }^{61}$, W. Ehrenfeld ${ }^{41}$, T. Ehrich ${ }^{99}$, T. Eifert ${ }^{29}$, G. Eigen $^{13}$, K. Einsweiler ${ }^{14}$, E. Eisenhandler ${ }^{75}$, T. Ekelof ${ }^{166}$, M. El Kacimi ${ }^{4}$, M. Ellert $^{166}$, S. Elles ${ }^{4}$, F. Ellinghaus ${ }^{81}$, K. Ellis ${ }^{75}$, N. Ellis $^{29}$, J. Elmsheuser ${ }^{98}$, M. Elsing ${ }^{29}$, D. Emeliyanov ${ }^{129}$, R. Engelmann ${ }^{148}$, A. Engl ${ }^{98}$, B. Epp ${ }^{62}$, A. Eppig ${ }^{87}$, J. Erdmann ${ }^{54}$, A. Ereditato ${ }^{16}$, D. Eriksson ${ }^{146 a}$, I. Ermoline ${ }^{88}$, J. Ernst ${ }^{1}$, M. Ernst $^{24}$, J. Ernwein ${ }^{136}$, D. Errede ${ }^{165}$, S. Errede ${ }^{165}$, E. Ertel ${ }^{81}$, M. Escalier ${ }^{115}$, C. Escobar ${ }^{167}$, X. Espinal Curull ${ }^{11}$, B. Esposito ${ }^{47}$, A.I. Etienvre ${ }^{136}$, E. Etzion ${ }^{153}$, H. Evans ${ }^{61}$, L. Fabbri ${ }^{19 a, 19 b}$, C. Fabre $^{29}$, K. Facius ${ }^{35}$, R.M. Fakhrutdinov ${ }^{128}$, S. Falciano ${ }^{132 a}$, Y. Fang ${ }^{172}$, M. Fanti ${ }^{89 a, 89 b}$, A. Farbin ${ }^{7}$, A. Farilla ${ }^{134 a}$, J. Farley ${ }^{148}$, T. Farooque ${ }^{158}$, S.M. Farrington ${ }^{18}$, P. Farthouat ${ }^{29}$, P. Fassnacht ${ }^{29}$, D. Fassouliotis ${ }^{8}$, B. Fatholahzadeh ${ }^{158}$, L. Fayard ${ }^{115}$, F. Fayette Fu $^{54}$,

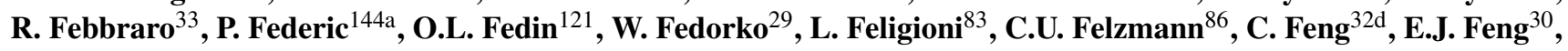




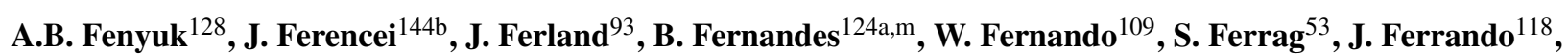
V. Ferrara ${ }^{41}$, A. Ferrari ${ }^{166}$, P. Ferrari ${ }^{105}$, R. Ferrari ${ }^{119 a}$, A. Ferrer ${ }^{167}$, M.L. Ferrer ${ }^{47}$, D. Ferrere ${ }^{49}$, C. Ferretti ${ }^{87}$, M. Fiascaris ${ }^{118}$, F. Fiedler ${ }^{81}$, A. Filipčič ${ }^{74}$, A. Filippas ${ }^{9}$, F. Filthaut ${ }^{104}$, M. Fincke-Keeler ${ }^{169}$, M.C.N. Fiolhais ${ }^{124 a, g}$, L. Fiorini ${ }^{11}$, A. Firan $^{39}$, G. Fischer ${ }^{41}$, M.J. Fisher ${ }^{109}$, M. Flechl ${ }^{48}$, I. Fleck ${ }^{141}$, J. Fleckner ${ }^{81}$, P. Fleischmann ${ }^{173}$, S. Fleischmann ${ }^{20}$, T. Flick ${ }^{174}$, L.R. Flores Castillo ${ }^{172}$, M.J. Flowerdew ${ }^{99}$, T.Fonseca Martin $^{76}$, J. Fopma ${ }^{118}$, A. Formica ${ }^{136}$, A. Forti ${ }^{82}$, D. Fortin ${ }^{159}$, D. Fournier ${ }^{115}$, A.J. Fowler ${ }^{44}$, K. Fowler $^{137}$, H. Fox ${ }^{71}$, P. Francavilla ${ }^{122 a, 122 b}$, S. Franchino ${ }^{119 a, 119 b}$, D. Francis ${ }^{29}$, M. Franklin ${ }^{57}$, S. Franz ${ }^{29}$, M. Fraternali ${ }^{119 a, 119 b}$, S. Fratina ${ }^{120}$, J. Freestone ${ }^{82}$, S.T. French ${ }^{27}$, R. Froeschl ${ }^{29}$, D. Froidevaux ${ }^{29}$, J.A. Frost ${ }^{27}$, C. Fukunaga ${ }^{156}$, E. Fullana Torregrosa ${ }^{5}$, J. Fuster ${ }^{167}$, C. Gabaldon ${ }^{80}$, O. Gabizon ${ }^{171}$, T. Gadfort ${ }^{24}$, S. Gadomski ${ }^{49}$, G. Gagliardi ${ }^{50 a, 50 b}$, P. Gagnon ${ }^{61}$, C. Galea ${ }^{98}$, E.J. Gallas ${ }^{118}$, V. Gallo ${ }^{16}$, B.J. Gallop ${ }^{129}$, P. Gallus ${ }^{125}$, E. Galyaev ${ }^{40}$, K.K. Gan ${ }^{109}$, Y.S. Gao ${ }^{143, n}$, A. Gaponenko ${ }^{14}$, M. Garcia-Sciveres ${ }^{14}$, C. García ${ }^{167}$, J.E. García Navarro ${ }^{49}$, R.W. Gardner ${ }^{30}$, N. Garelli ${ }^{29}$, H. Garitaonandia ${ }^{105}$, V. Garonne ${ }^{29}$, C. Gatti ${ }^{47}$, G. Gaudio ${ }^{119 a}$, V. Gautard ${ }^{136}$, P. Gauzzi ${ }^{132 a, 132 b}$, I.L. Gavrilenko ${ }^{94}$, C. Gay ${ }^{168}$, G. Gaycken $^{20}$, E.N. Gazis ${ }^{9}$, P. Ge ${ }^{32 \mathrm{~d}}$, C.N.P. Gee ${ }^{129}$, Ch. Geich-Gimbel $^{20}$, K. Gellerstedt ${ }^{146 a, 146 b}$, C. Gemme $^{50 a}$, M.H. Genest ${ }^{98}$, S. Gentile ${ }^{132 a, 132 b}$, F. Georgatos ${ }^{9}$, S. George ${ }^{76}$, A. Gershon ${ }^{153}$, H. Ghazlane ${ }^{135 d}$, N. Ghodbane ${ }^{33}$, B. Giacobbe ${ }^{19 a}$, S. Giagu ${ }^{132 a, 132 b}$, V. Giakoumopoulou ${ }^{8}$, V. Giangiobbe ${ }^{122 a, 122 b}$, F. Gianottii ${ }^{29}$, B. Gibbard ${ }^{24}$, A. Gibson ${ }^{158}$, S.M. Gibson ${ }^{118}$, L.M. Gilbert ${ }^{118}$, M. Gilchriese ${ }^{14}$, V. Gilewsky ${ }^{91}$, D.M. Gingrich ${ }^{2, o}$, J. Ginzburg ${ }^{153}$, N. Giokaris ${ }^{8}$, M.P. Giordani ${ }^{164 a, 164 c}$, R. Giordano ${ }^{102 a, 102 b}$, F.M. Giorgi ${ }^{15}$, P. Giovannini ${ }^{99}$, P.F. Giraud ${ }^{136}$, P. Girtler ${ }^{62}$, D. Giugni ${ }^{89}$, P. Giusti ${ }^{19 a}$, B.K. Gjelsten ${ }^{117}$, L.K. Gladilin ${ }^{97}$, C. Glasman ${ }^{80}$, A. Glazov ${ }^{41}$, K.W. Glitza ${ }^{174}$, G.L. Glonti ${ }^{65}$, J. Godfrey ${ }^{142}$, J. Godlewski ${ }^{29}$, M. Goebel ${ }^{41}$, T. Göpfert ${ }^{43}$, C. Goeringer ${ }^{81}$, C. Gössling ${ }^{42}$, T. Göttfert ${ }^{99}$, V. Goggi ${ }^{119 a, 119 b, p}$, S. Goldfarb ${ }^{87}$, D. Goldin ${ }^{39}$, T. Golling ${ }^{175}$, A. Gomes ${ }^{124 a, q}$, L.S. Gomez Fajardo ${ }^{41}$, R. Gonçalo ${ }^{76}$, L. Gonella ${ }^{20}$, C. Gong ${ }^{32 b}$, S. González de la Hoz ${ }^{167}$, M.L. Gonzalez Silva ${ }^{26}$, S. Gonzalez-Sevilla ${ }^{49}$, J.J. Goodson ${ }^{148}$, L. Goossens ${ }^{29}$, H.A. Gordon ${ }^{24}$, I. Gorelov ${ }^{103}$, G. Gorfine ${ }^{174}$, B. Gorini ${ }^{29}$, E. Gorini ${ }^{72 a, 72 b}$, A. Gorišek ${ }^{74}$, E. Gornicki $^{38}$, B. Gosdzik ${ }^{41}$, M. Gosselink ${ }^{105}$, M.I. Gostkin ${ }^{65}$, I. Gough Eschrich ${ }^{163}$, M. Gouighri ${ }^{135 a}$, D. Goujdami ${ }^{135 a}$, M.P. Goulette ${ }^{49}$, A.G. Goussiou ${ }^{138}$, C. Goy ${ }^{4}$, I. Grabowska-Bold ${ }^{163, r}$, P. Grafström ${ }^{29}$, K.-J. Grahn ${ }^{147}$, S. Grancagnolo ${ }^{15}$, V. Grassi ${ }^{148}$, V. Gratchev ${ }^{121}$, N. Grau ${ }^{34}$, H.M. Gray ${ }^{34, \mathrm{~s}}$, J.A. Gray ${ }^{148}$, E. Graziani ${ }^{134 a}$, B. Green ${ }^{76}$, T. Greenshaw ${ }^{73}$, Z.D. Greenwood ${ }^{24, t}$, I.M. Gregor ${ }^{41}$, P. Grenier ${ }^{143}$, E. Griesmayer ${ }^{46}$, J. Griffiths ${ }^{138}$, N. Grigalashvili ${ }^{65}$, A.A. Grillo ${ }^{137}$, K. Grimm ${ }^{148}$, S. Grinstein ${ }^{11}$, Y.V. Grishkevich ${ }^{97}$, M. Groh ${ }^{99}$, M. Groll ${ }^{81}$, E. Gross ${ }^{171}$, J. Grosse-Knetter ${ }^{54}$, J. Groth-Jensen ${ }^{79}$, K. Grybel ${ }^{141}$, C. Guicheney ${ }^{33}$, A. Guida ${ }^{72 a, 72 b}$, T. Guillemin ${ }^{4}$, H. Guler ${ }^{85, u}$, J. Gunther ${ }^{125}$, B. Guo ${ }^{158}$, L. Gurriana ${ }^{124 a}$, Y. Gusakov ${ }^{65}$, A. Gutierrez ${ }^{93}$,

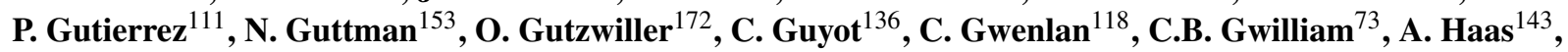

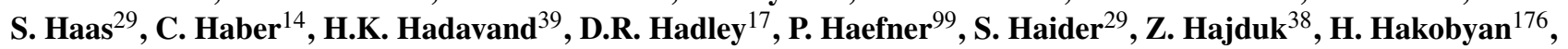

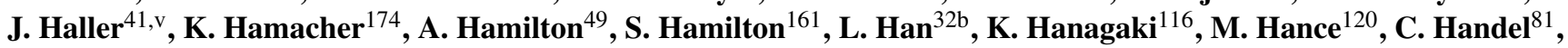
P. Hanke ${ }^{58 a}$, J.R. Hansen ${ }^{35}$, J.B. Hansen ${ }^{35}$, J.D. Hansen ${ }^{35}$, P.H. Hansen ${ }^{35}$, T. Hansl-Kozanecka ${ }^{137}$, P. Hansson ${ }^{143}$, K. Hara ${ }^{160}$, G.A. Hare ${ }^{137}$, T. Harenberg ${ }^{174}$, R.D. Harrington ${ }^{21}$, O.M. Harris ${ }^{138}$, K. Harrison ${ }^{17}$, J. Hartert ${ }^{48}$, F. Hartjes ${ }^{105}$, A. Harvey ${ }^{56}$, S. Hasegawa ${ }^{101}$, Y. Hasegawa ${ }^{140}$, S. Hassani ${ }^{136}$, S. Haug ${ }^{16}$, M. Hauschild ${ }^{29}$, R. Hauser ${ }^{88}$, M. Havranek ${ }^{125}$, C.M. Hawkes ${ }^{17}$, R.J. Hawkings ${ }^{29}$, T. Hayakawa ${ }^{67}$, H.S. Hayward ${ }^{73}$, S.J. Haywood ${ }^{129}$, S.J. Head ${ }^{82}$, V. Hedberg ${ }^{79}$, L. Heelan ${ }^{28}$, S. Heim ${ }^{88}$, B. Heinemann ${ }^{14}$, S. Heisterkamp ${ }^{35}$, L. Helary ${ }^{4}$, M. Heller ${ }^{115}$, S. Hellman ${ }^{146 a, 146 b}$, C. Helsens ${ }^{11}$, T. Hemperek ${ }^{20}$, R.C.W. Henderson ${ }^{71}$, M. Henke ${ }^{58 a}$, A. Henrichs ${ }^{54}$, A.M. Henriques Correia ${ }^{29}$, S. Henrot-Versille ${ }^{115}$, C. Hensel $^{54}$, T. Henß ${ }^{174}$, Y. Hernández Jiménez ${ }^{167}$, A.D. Hershenhorn ${ }^{152}$, G. Herten ${ }^{48}$, R. Hertenberger ${ }^{98}$, L. Hervas ${ }^{29}$, N.P. Hessey ${ }^{105}$, E. Higón-Rodriguez ${ }^{167}$, J.C. Hill ${ }^{27}$, K.H. Hiller ${ }^{41}$, S. Hillert ${ }^{146 a}$,146b, S.J. Hillier ${ }^{17}$, I. Hinchliffe ${ }^{14}$, E. Hines ${ }^{120}$, M. Hirose ${ }^{116}$, F. Hirsch ${ }^{42}$,

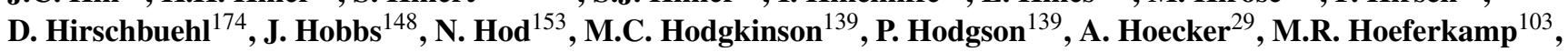
J. Hoffman ${ }^{39}$, D. Hoffmann ${ }^{83}$, M. Hohlfeld ${ }^{81}$, D. Hollander ${ }^{30}$, T. Holy ${ }^{127}$, J.L. Holzbauer ${ }^{88}$, Y. Homma ${ }^{67}$,

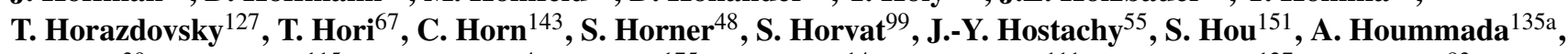

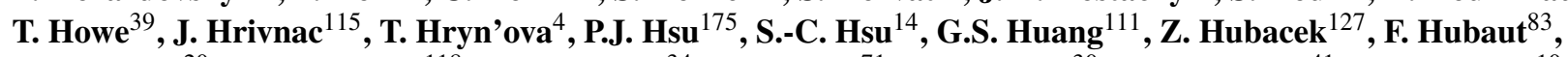

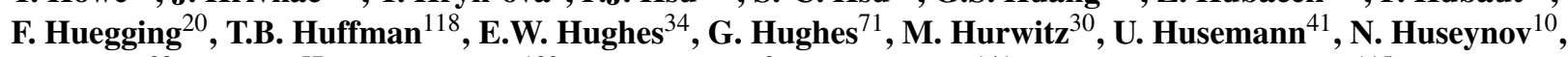
J. Huston ${ }^{88}$, J. Huth ${ }^{57}$, G. Iacobucci ${ }^{102 a}$, G. Iakovidis ${ }^{9}$, I. Ibragimov ${ }^{141}$, L. Iconomidou-Fayard ${ }^{115}$, J. Idarraga ${ }^{159 b}$, P. Iengo $^{4}$, O. Igonkina ${ }^{105}$, Y. Ikegami ${ }^{66}$, M. Ikeno ${ }^{66}$, Y. Ilchenko ${ }^{39}$, D. Iliadis ${ }^{154}$, T. Ince ${ }^{20}$, P. Ioannou ${ }^{8}$, M. Iodice ${ }^{134 a}$, A. Irles Quiles ${ }^{167}$, A. Ishikawa ${ }^{67}$, M. Ishino ${ }^{66}$, R. Ishmukhametov ${ }^{39}$, T. Isobe ${ }^{155}$, C. Issever ${ }^{118}$, S. Istin ${ }^{18 a}$, Y. Itoh ${ }^{101}$, A.V. Ivashin ${ }^{128}$, W. Iwanski ${ }^{38}$, H. Iwasaki ${ }^{66}$, J.M. Izen $^{40}$, V. Izzo ${ }^{102 a}$, B. Jackson ${ }^{120}$, J.N. Jackson ${ }^{73}$, P. Jackson ${ }^{143}$, M.R. Jaekel ${ }^{29}$, V. Jain ${ }^{61}$, K. Jakobs ${ }^{48}$, S. Jakobsen ${ }^{35}$, J. Jakubek ${ }^{127}$, D.K. Jana ${ }^{111}$, E. Jankowski ${ }^{158}$, E. Jansen ${ }^{77}$, A. Jantsch $^{99}$, M. Janus ${ }^{48}$, G. Jarlskog ${ }^{79}$, L. Jeanty ${ }^{57}$, I. Jen-La Plante ${ }^{30}$, P. Jenni ${ }^{29}$, P. Jež ${ }^{35}$, S. Jézéquel ${ }^{4}$, W. Ji ${ }^{79}$, 
J. Jia ${ }^{148}$, Y. Jiang ${ }^{32 b}$, M. Jimenez Belenguer ${ }^{29}$, S. Jin ${ }^{32 a}$, O. Jinnouchii ${ }^{157}$, D. Joffe ${ }^{39}$, M. Johansen ${ }^{146 a, 146 b}$, K.E. Johansson ${ }^{146 a}$, P. Johansson ${ }^{139}$, S. Johnert ${ }^{41}$, K.A. Johns ${ }^{6}$, K. Jon-And ${ }^{146 a, 146 b}$, G. Jones ${ }^{82}$, R.W.L. Jones ${ }^{71}$, T.J. Jones ${ }^{73}$, P.M. Jorge ${ }^{124 a, b}$, J. Joseph ${ }^{14}$, V. Juranek ${ }^{125}$, P. Jussel ${ }^{62}$, V.V. Kabachenko ${ }^{128}$, M. Kaci ${ }^{167}$, A. Kaczmarska ${ }^{38}$, M. Kado ${ }^{115}$, H. Kagan ${ }^{109}$, M. Kagan ${ }^{57}$, S. Kaiser ${ }^{99}$, E. Kajomovitz ${ }^{152}$, S. Kalinin ${ }^{174}$, L.V. Kalinovskaya ${ }^{65}$, S. Kama ${ }^{41}$, N. Kanaya ${ }^{155}$, M. Kaneda ${ }^{155}$, V.A. Kantserov ${ }^{96}$, J. Kanzaki ${ }^{66}$, B. Kaplan ${ }^{175}$, A. Kapliy ${ }^{30}$, J. Kaplon ${ }^{29}$, D. Kar ${ }^{43}$, M. Karagounis ${ }^{20}$, M. Karagoz Unel ${ }^{118}$, M. Karnevskiy ${ }^{41}$, V. Kartvelishvili ${ }^{71}$, A.N. Karyukhin ${ }^{128}$, L. Kashif ${ }^{57}$, A. Kasmi ${ }^{39}$, R.D. Kass ${ }^{109}$, A. Kastanas ${ }^{13}$, M. Kastoryano ${ }^{175}$, M. Kataoka ${ }^{4}$, Y. Kataoka ${ }^{155}$, E. Katsoufis 9 , J. Katzy ${ }^{41}$, V. Kaushik ${ }^{6}$, K. Kawagoe ${ }^{67}$, T. Kawamoto ${ }^{155}$, G. Kawamura ${ }^{81}$, M.S. Kayl ${ }^{105}$, F. Kayumov ${ }^{94}$, V.A. Kazanin ${ }^{107}$, M.Y. Kazarinov ${ }^{65}$, J.R. Keates ${ }^{82}$, R. Keeler ${ }^{169}$, P.T. Keener ${ }^{120}$, R. Kehoe ${ }^{39}$, M. Keil ${ }^{54}$, G.D. Kekelidze ${ }^{65}$, M. Kelly ${ }^{82}$, M. Kenyon ${ }^{53}$, O. Kepka ${ }^{125}$, N. Kerschen ${ }^{29}$, B.P. Kerševan ${ }^{74}$, S. Kersten ${ }^{174}$, K. Kessoku ${ }^{155}$, M. Khakzad ${ }^{28}$, F. Khalil-zada ${ }^{10}$, H. Khandanyan ${ }^{165}$, A. Khanov ${ }^{112}$, D. Kharchenko ${ }^{65}$, A. Khodinov ${ }^{148}$, A. Khomich ${ }^{58 a}$, G. Khoriauli ${ }^{20}$, N. Khovanskiy ${ }^{65}$, V. Khovanskiy ${ }^{95}$, E. Khramov ${ }^{65}$, J. Khubua ${ }^{51}$,

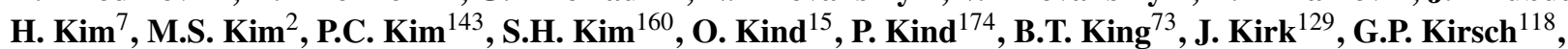

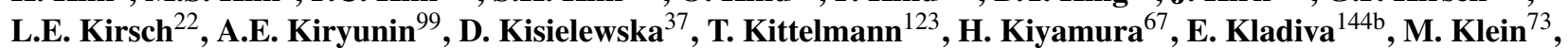
U. Klein $^{73}$, K. Kleinknecht ${ }^{81}$, M. Klemetti ${ }^{85}$, A. Klier ${ }^{171}$, A. Klimentov ${ }^{24}$, R. Klingenberg ${ }^{42}$, E.B. Klinkby ${ }^{44}$, T. Klioutchnikova ${ }^{29}$, P.F. Klok ${ }^{104}$, S. Klous ${ }^{105}$, E.-E. Kluge ${ }^{58 a}$, T. Kluge ${ }^{73}$, P. Kluit ${ }^{105}$, M. Klute ${ }^{54}$, S. Kluth ${ }^{99}$, N.S. Knecht ${ }^{158}$, E. Kneringer ${ }^{62}$, B.R. Ko ${ }^{44}$, T. Kobayashi ${ }^{155}$, M. Kobel ${ }^{43}$, B. Koblitz ${ }^{29}$, M. Kocian ${ }^{143}$, A. Kocnar ${ }^{113}$, P. Kodys ${ }^{126}$, K. Köneke ${ }^{41}$, A.C. König ${ }^{104}$, S. Koenig ${ }^{81}$, L. Köpke ${ }^{81}$, F. Koetsveld ${ }^{104}$, P. Koevesarki ${ }^{20}$, T. Koffas ${ }^{29}$, E. Koffeman ${ }^{105}$, F. Kohn ${ }^{54}$, Z. Kohout ${ }^{127}$, T. Kohriki ${ }^{66}$, H. Kolanoski ${ }^{15}$, V. Kolesnikov ${ }^{65}$, I. Koletsou ${ }^{4}$, J. Koll ${ }^{88}$, D. Kollar $^{29}$, S. Kolos ${ }^{163, w}$, S.D. Kolya ${ }^{82}$, A.A. Komar ${ }^{94}$, J.R. Komaragiri ${ }^{142}$, T. Kondo ${ }^{66}$, T. Kono ${ }^{41, x}$, R. Konoplich ${ }^{108}$, S.P. Konovalov ${ }^{94}$, N. Konstantinidis ${ }^{77}$, S. Koperny ${ }^{37}$, K. Korcyl ${ }^{38}$, K. Kordas ${ }^{154}$, A. Korn ${ }^{14}$, I. Korolkov $^{11}$, E.V. Korolkova ${ }^{139}$, V.A. Korotkov ${ }^{128}$, O. Kortner ${ }^{99}$, P. Kostka ${ }^{41}$, V.V. Kostyukhin ${ }^{20}$, S. Kotov ${ }^{99}$, V.M. Kotov ${ }^{65}$, K.Y. Kotov ${ }^{107}$, C. Kourkoumelis ${ }^{8}$, A. Koutsman ${ }^{105}$, R. Kowalewski ${ }^{169}$, H. Kowalski ${ }^{41}$, T.Z. Kowalski ${ }^{37}$, W. Kozanecki ${ }^{136}$, A.S. Kozhin ${ }^{128}$, V. Kral ${ }^{127}$, V.A. Kramarenko ${ }^{97}$, G. Kramberger ${ }^{74}$,

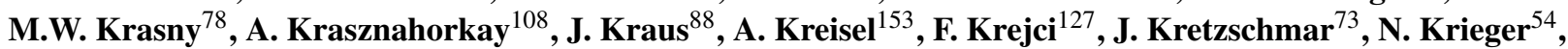
P. Krieger ${ }^{158}$, K. Kroeninger ${ }^{54}$, H. Kroha ${ }^{99}$, J. Kroll ${ }^{120}$, J. Kroseberg ${ }^{20}$, J. Krstic ${ }^{12 a}$, U. Kruchonak ${ }^{65}$, H. Krüger ${ }^{20}$, Z.V. Krumshteyn ${ }^{65}$, T. Kubota ${ }^{155}$, S. Kuehn ${ }^{48}$, A. Kugel ${ }^{58 c}$, T. Kuhl ${ }^{174}$, D. Kuhn ${ }^{62}$, V. Kukhtin ${ }^{65}$, Y. Kulchitsky ${ }^{90}$, S. Kuleshov ${ }^{31 b}$, C. Kummer ${ }^{98}$, M. Kuna ${ }^{83}$, J. Kunkle ${ }^{120}$, A. Kupco ${ }^{125}$, H. Kurashige ${ }^{67}$, M. Kurata ${ }^{160}$, Y.A. Kurochkin ${ }^{90}$, V. Kus ${ }^{125}$, R. Kwee ${ }^{15}$, A. La Rosa ${ }^{29}$, L. La Rotonda ${ }^{36 a, 36 b}$, J. Labbe ${ }^{4}$, C. Lacasta ${ }^{167}$,

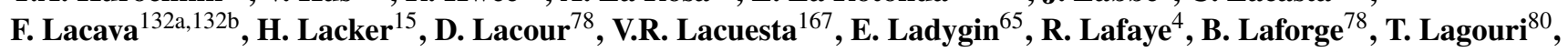

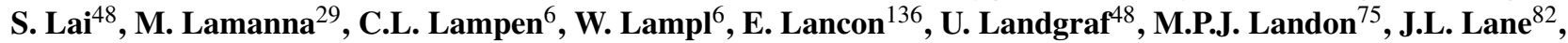

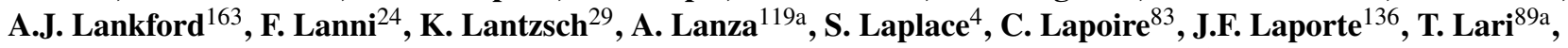
A. Larner ${ }^{18}$, M. Lassnig ${ }^{29}$, P. Laurelli ${ }^{47}$, W. Lavrijsen ${ }^{14}$, P. Laycock ${ }^{73}$, A.B. Lazarev $^{65}$, A. Lazzaro ${ }^{89 a, 89 b}$,

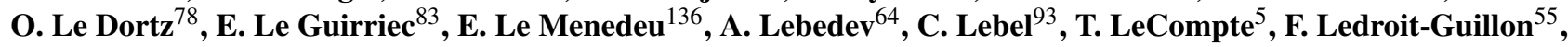

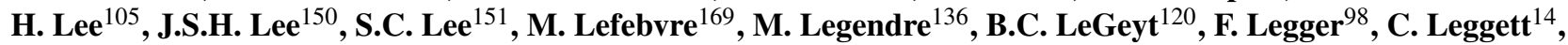
M. Lehmacher ${ }^{20}$, G. Lehmann Miotto ${ }^{29}$, X. Lei ${ }^{6}$, R. Leitner ${ }^{126}$, D. Lellouch ${ }^{171}$, J. Lellouch ${ }^{78}$, V. Lendermann ${ }^{58 a}$, K.J.C. Leney ${ }^{73}$, T. Lenz ${ }^{174}$, G. Lenzen ${ }^{174}$, B. Lenzi ${ }^{136}$, K. Leonhardt ${ }^{43}$, C. Leroy ${ }^{93}$, J.-R. Lessard ${ }^{169}$, C.G. Lester ${ }^{27}$, A. Leung Fook Cheong ${ }^{172}$, J. Levêque ${ }^{83}$, D. Levin ${ }^{87}$, L.J. Levinson ${ }^{171}$, M. Leyton ${ }^{15}$, H. Li ${ }^{172}$, X. Li ${ }^{87}$, Z. Liang ${ }^{39}$, Z. Liang ${ }^{151, y}$, B. Liberti ${ }^{133 a}$, P. Lichard ${ }^{29}$, M. Lichtnecker ${ }^{98}$, K. Lie ${ }^{165}$, W. Liebig ${ }^{105}$, J.N. Lilley ${ }^{17}$, A. Limosani ${ }^{86}$, M. Limper ${ }^{63}$, S.C. Lin ${ }^{151}$, J.T. Linnemann ${ }^{88}$, E. Lipeles ${ }^{120}$, L. Lipinsky ${ }^{125}$, A. Lipniacka ${ }^{13}$, T.M. Liss ${ }^{165}$,

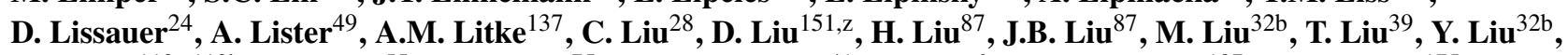
M. Livan ${ }^{119 a, 119 b}$, A. Lleres ${ }^{55}$, S.L. Lloyd ${ }^{75}$, E. Lobodzinska ${ }^{41}$, P. Loch ${ }^{6}$, W.S. Lockman ${ }^{137}$, S. Lockwitz ${ }^{175}$, T. Loddenkoetter ${ }^{20}$, F.K. Loebinger ${ }^{82}$, A. Loginov ${ }^{175}$, C.W. Loh ${ }^{168}$, T. Lohse ${ }^{15}$, K. Lohwasser ${ }^{48}$, M. Lokajicek ${ }^{125}$,

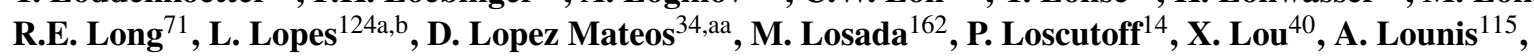

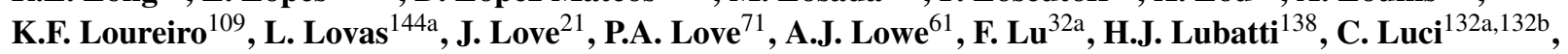

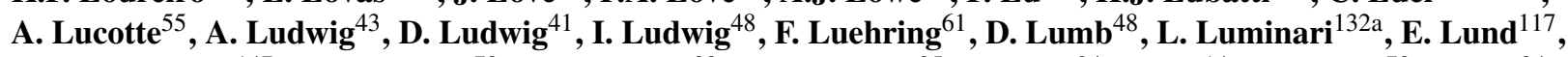
B. Lund-Jensen ${ }^{147}$, B. Lundberg ${ }^{79}$, J. Lundberg ${ }^{29}$, J. Lundquist ${ }^{35}$, D. Lynn ${ }^{24}$, J. Lys ${ }^{14}$, E. Lytken ${ }^{79}$, H. Ma ${ }^{24}$, L.L. Ma ${ }^{172}$, J.A. Macana Goia ${ }^{93}$, G. Maccarrone ${ }^{47}$, A. Macchiolo ${ }^{99}$, B. Maček ${ }^{74}$, J. Machado Miguens ${ }^{124 a, b}$, R. Mackeprang ${ }^{35}$, R.J. Madaras $^{14}$, W.F. Mader ${ }^{43}$, R. Maenner ${ }^{58 c}$, T. Maeno ${ }^{24}$, P. Mättig ${ }^{174}$, S. Mättig ${ }^{41}$, P.J. Magalhaes Martins ${ }^{124 a, g}$, E. Magradze ${ }^{51}$, Y. Mahalalel ${ }^{153}$, K. Mahboubi $^{48}$, A. Mahmood $^{1}$, C. Maiani ${ }^{132 a, 132 b}$,

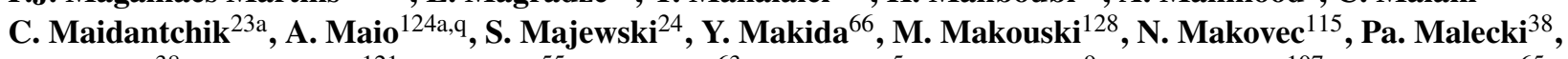
P. Malecki ${ }^{38}$, V.P. Maleev ${ }^{121}$, F. Malek ${ }^{55}$, U. Mallik ${ }^{63}$, D. Malon ${ }^{5}$, S. Maltezos ${ }^{9}$, V. Malyshev ${ }^{107}$, S. Malyukov ${ }^{65}$, 
M. Mambelli ${ }^{30}$, R. Mameghani ${ }^{98}$, J. Mamuzic ${ }^{41}$, L. Mandelli ${ }^{89 a}$, I. Mandic ${ }^{74}$, R. Mandrysch $^{15}$, J. Maneira ${ }^{124 a}$, P.S. Mangeard ${ }^{88}$, L. Manhaes de Andrade Filho ${ }^{23 a}$, I.D. Manjavidze ${ }^{65}$, P.M. Manning ${ }^{137}$,

A. Manousakis-Katsikakis ${ }^{8}$, B. Mansoulie ${ }^{136}$, A. Mapelli ${ }^{29}$, L. Mapelli ${ }^{29}$, L. March ${ }^{80}$, J.F. Marchand ${ }^{4}$, F. Marchese ${ }^{133 a, 133 b}$, G. Marchiori $^{78}$, M. Marcisovsky ${ }^{125}$, C.P. Marino ${ }^{61}$, F. Marroquim $^{23 a}$, Z. Marshall $^{34, a a}$,

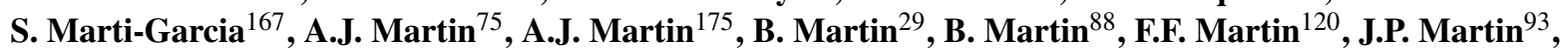
T.A. Martin ${ }^{17}$, B. Martin dit Latour ${ }^{49}$, M. Martinez ${ }^{11}$, V. Martinez Outschoorn ${ }^{57}$, A.C. Martyniuk ${ }^{82}$, F. Marzano ${ }^{132 a}$, A. Marzin $^{136}$, L. Masetti $^{20}$, T. Mashimo ${ }^{155}$, R. Mashinistov ${ }^{96}$, J. Masik ${ }^{82}$, A.L. Maslennikov ${ }^{107}$, I. Massa ${ }^{19 a, 19 b}$, N. Massol ${ }^{4}$, A. Mastroberardino ${ }^{36 a, 36 b}$, T. Masubuchi ${ }^{155}$, P. Matricon ${ }^{115}$, H. Matsunaga $^{155}$, T. Matsushita ${ }^{67}$, C. Mattravers ${ }^{118, a b}$, S.J. Maxfield ${ }^{73}$, A. Mayne ${ }^{139}$, R. Mazini ${ }^{151}$, M. Mazur ${ }^{48}$, J. Mc Donald ${ }^{85}$, S.P. Mc Kee ${ }^{87}$, A. McCarn ${ }^{165}$, R.L. McCarthy ${ }^{148}$, N.A. McCubbin ${ }^{129}$, K.W. McFarlane ${ }^{56}$, H. McGlone ${ }^{53}$, G. Mchedlidze $^{51}$, S.J. McMahon ${ }^{129}$, R.A. McPherson ${ }^{169, j}$, A. Meade ${ }^{84}$, J. Mechnich ${ }^{105}$, M. Mechtel ${ }^{174}$, M. Medinnis ${ }^{41}$, R. Meera-Lebbai ${ }^{111}$, T.M. Meguro ${ }^{116}$, S. Mehlhase ${ }^{41}$, A. Mehta $^{73}$, K. Meier ${ }^{58 a}$, B. Meirose ${ }^{48}$, C. Melachrinos ${ }^{30}$, B.R. Mellado Garcia ${ }^{172}$, L. Mendoza Navas ${ }^{162}$, Z. Meng ${ }^{151, \text { ac }}$, S. Menke $^{99}$, E. Meoni ${ }^{11}$, P. Mermod ${ }^{118}$, L. Merola ${ }^{102 a, 102 b}$, C. Meroni ${ }^{89 a}$, F.S. Merritt ${ }^{30}$, A.M. Messina ${ }^{29}$, J. Metcalfe $^{103}$, A.S. Mete ${ }^{64}$, J.-P. Meyer ${ }^{136}$, J. Meyer ${ }^{173}$, J. Meyer ${ }^{54}$, T.C. Meyer $^{29}$, W.T. Meyer ${ }^{64}$, J. Miao ${ }^{32 d}$, S. Michal ${ }^{29}$, L. Micu ${ }^{25 a}$, R.P. Middleton ${ }^{129}$, S. Migas ${ }^{73}$, L. Mijović ${ }^{74}$, G. Mikenberg ${ }^{171}$, M. Mikestikova ${ }^{125}$, M. Mikuž ${ }^{74}$, D.W. Miller ${ }^{143}$, M. Miller ${ }^{30}$, W.J. Mills ${ }^{168}$, C.M. Mills ${ }^{57}$, A. Milov ${ }^{171}$, D.A. Milstead ${ }^{146 a, 146 b}$, D. Milstein ${ }^{171}$, A.A. Minaenko ${ }^{128}$, M. Miñano ${ }^{167}$, I.A. Minashvili ${ }^{65}$, A.I. Mincer ${ }^{108}$, B. Mindur $^{37}$, M. Mineev ${ }^{65}$, Y. Ming ${ }^{130}$, L.M. Mir ${ }^{11}$, G. Mirabelli ${ }^{132 a}$, S. Misawa ${ }^{24}$, A. Misiejuk ${ }^{76}$, J. Mitrevski ${ }^{137}$, V.A. Mitsou ${ }^{167}$, P.S. Miyagawa ${ }^{82}$, J.U. Mjörnmark ${ }^{79}$, T. Moa ${ }^{146 a, 146 b}$, S. Moed ${ }^{57}$, V. Moeller ${ }^{27}$, K. Mönig ${ }^{41}$, N. Möser ${ }^{20}$, W. Mohr ${ }^{48}$, S. Mohrdieck-Möck ${ }^{99}$, R. Moles-Valls ${ }^{167}$, J. Molina-Perez ${ }^{29}$, J. Monk ${ }^{77}$, E. Monnier $^{83}$, S. Montesano ${ }^{89 a}$, 89b, F. Monticelli ${ }^{70}$, R.W. Moore ${ }^{2}$, C. Mora Herrera ${ }^{49}$, A. Moraes $^{53}$, A. Morais ${ }^{124 a, b}$, J. Morel ${ }^{54}$, G. Morello ${ }^{36 a, 36 b}$, D. Moreno ${ }^{162}$, M. Moreno Llácer ${ }^{167}$, P. Morettini ${ }^{50 a}$, M. Morii ${ }^{57}$, A.K. Morley ${ }^{86}$, G. Mornacchi $^{29}$, S.V. Morozov ${ }^{96}$, J.D. Morris $^{75}$, H.G. Moser ${ }^{99}$, M. Mosidze $^{51}$, J. Moss ${ }^{109}$, R. Mount ${ }^{143}$, E. Mountricha ${ }^{136}$, S.V. Mouraviev ${ }^{94}$, E.J.W. Moyse ${ }^{84}$, M. Mudrinic ${ }^{12 b}$, F. Mueller ${ }^{58 a}$, J. Mueller ${ }^{123}$, K. Mueller ${ }^{20}$, T.A. Müller ${ }^{98}$, D. Muenstermann ${ }^{42}$, A. Muir ${ }^{168}$, Y. Munwes ${ }^{153}$, R. Murillo Garcia ${ }^{163}$, W.J. Murray ${ }^{129}$, I. Mussche ${ }^{105}$, E. Musto ${ }^{102 a, 102 b}$, A.G. Myagkov ${ }^{128}$, M. Myska ${ }^{125}$, J. Nadal ${ }^{11}$, K. Nagai ${ }^{160}$, K. Nagano ${ }^{66}$, Y. Nagasaka ${ }^{60}$, A.M. Nairz ${ }^{29}$, K. Nakamura ${ }^{155}$, I. Nakano ${ }^{110}$, H. Nakatsuka ${ }^{67}$, G. Nanava ${ }^{20}$, A. Napier ${ }^{161}$, M. Nash ${ }^{77, a d}$, N.R. Nation ${ }^{21}$, T. Nattermann ${ }^{20}$, T. Naumann ${ }^{41}$, G. Navarro ${ }^{162}$,

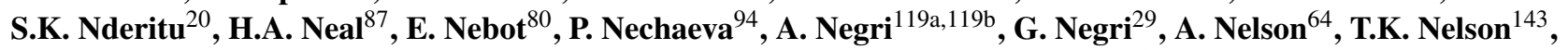
S. Nemecek ${ }^{125}$, P. Nemethy ${ }^{108}$, A.A. Nepomuceno ${ }^{23 a}$, M. Nessi ${ }^{29}$, M.S. Neubauer ${ }^{165}$, A. Neusiedl ${ }^{81}$, R.M. Neves ${ }^{108}$, P. Nevski ${ }^{24}$, F.M. Newcomer ${ }^{120}$, R.B. Nickerson ${ }^{118}$, R. Nicolaidou ${ }^{136}$, L. Nicolas ${ }^{139}$, G. Nicoletti $^{47}$, B. Nicquevert ${ }^{29}$, F. Niedercorn ${ }^{115}$, J. Nielsen ${ }^{137}$, A. Nikiforov ${ }^{15}$, K. Nikolaev $^{65}$, I. Nikolic-Audit $^{78}$, K. Nikolopoulos ${ }^{8}$, H. Nilsen ${ }^{48}$, P. Nilsson ${ }^{7}$, A. Nisati ${ }^{132 a}$, T. Nishiyama ${ }^{67}$, R. Nisius ${ }^{99}$, L. Nodulman ${ }^{5}$, M. Nomachi ${ }^{116}$, I. Nomidis ${ }^{154}$, M. Nordberg ${ }^{29}$, B. Nordkvist ${ }^{146 a, 146 b}$, D. Notz ${ }^{41}$, J. Novakova ${ }^{126}$, M. Nozaki ${ }^{66}$, M. Nožička ${ }^{41}$, I.M. Nugent ${ }^{159 a}$, A.-E. Nuncio-Quiroz ${ }^{20}$, G. Nunes Hanninger ${ }^{20}$, T. Nunnemann ${ }^{98}$, E. Nurse ${ }^{77}$, D.C. O'Neil ${ }^{142}$, V. O'Shea ${ }^{53}$,

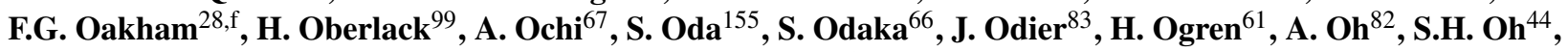
C.C. Ohm ${ }^{146 a, 146 b}$, T. Ohshima ${ }^{101}$, H. Ohshita ${ }^{140}$, T. Ohsugi ${ }^{59}$, S. Okada ${ }^{67}$, H. Okawa ${ }^{163}$, Y. Okumura ${ }^{101}$, T. Okuyama ${ }^{155}$, A.G. Olchevski ${ }^{65}$, M. Oliveira ${ }^{124 a, g}$, D. Oliveira Damazio ${ }^{24}$, E. Oliveira Garcia ${ }^{167}$, D. Olivito ${ }^{120}$, A. Olszewski ${ }^{38}$, J. Olszowska ${ }^{38}$, C. Omachi ${ }^{67, a e}$, A. Onofre ${ }^{124 a, a f}$, P.U.E. Onyisi ${ }^{30}$, C.J. Oram ${ }^{159 a}$, M.J. Oreglia ${ }^{30}$, Y. Oren ${ }^{153}$, D. Orestano ${ }^{134 a, 134 b}$, I. Orlov ${ }^{107}$, C. Oropeza Barrera ${ }^{53}$, R.S. Orr ${ }^{158}$, E.O. Ortega ${ }^{130}$, B. Osculati ${ }^{50 a, 50 b}$, R. Ospanov ${ }^{120}$, C. Osuna ${ }^{11}$, J.P Ottersbach ${ }^{105}$, F. Ould-Saada ${ }^{117}$, A. Ouraou ${ }^{136}$, Q. Ouyang ${ }^{32 a}$, M. Owen ${ }^{82}$, S. Owen ${ }^{139}$, A. Oyarzun ${ }^{316}$, V.E. Ozcan ${ }^{77}$, K. Ozone ${ }^{66}$, N. Ozturk ${ }^{7}$, A. Pacheco Pages ${ }^{11}$, C. Padilla Aranda ${ }^{11}$, E. Paganis ${ }^{139}$, C. Pahl ${ }^{63}$, F. Paige ${ }^{24}$, K. Pajchel ${ }^{117}$, S. Palestini ${ }^{29}$, D. Pallin ${ }^{33}$, A. Palma ${ }^{124 a, b}$, J.D. Palmer ${ }^{17}$, Y.B. Pan ${ }^{172}$, E. Panagiotopoulou ${ }^{9}$, B. Panes ${ }^{31 a}$, N. Panikashvili ${ }^{87}$, S. Panitkin ${ }^{24}$, D. Pantea ${ }^{25 a}$, M. Panuskova ${ }^{125}$, V. Paolone ${ }^{123}$, Th.D. Papadopoulou ${ }^{9}$, S.J. Park ${ }^{54}$, W. Park ${ }^{24, a g}$, M.A. Parker ${ }^{27}$, F. Parodi $^{50 a, 50 b}$, J.A. Parsons ${ }^{34}$, U. Parzefall ${ }^{48}$, E. Pasqualucci ${ }^{132 a}$, A. Passeri ${ }^{134 a}$, F. Pastore ${ }^{134 a, 134 b}$, Fr. Pastore ${ }^{29}$, G. Pásztor ${ }^{49}$,ah, S. Pataraia ${ }^{99}$, J.R. Pater ${ }^{82}$, S. Patricelli ${ }^{102 a, 102 b}$, T. Pauly ${ }^{29}$, L.S. Peak ${ }^{150}$, M. Pecsy ${ }^{144 a}$, M.I. Pedraza Morales ${ }^{172}$, S.V. Peleganchuk ${ }^{107}$, H. Peng ${ }^{172}$, A. Penson ${ }^{34}$, J. Penwell ${ }^{61}$, M. Perantoni ${ }^{23 a}$, K. Perez $^{34, a a}$, E. Perez Codina ${ }^{11}$, M.T. Pérez García-Estañ ${ }^{167}$, V. Perez Reale ${ }^{34}$, L. Perini ${ }^{89 a, 89 b}$, H. Pernegger ${ }^{29}$, R. Perrino ${ }^{72 a}$, S. Persembe ${ }^{3 a}$,

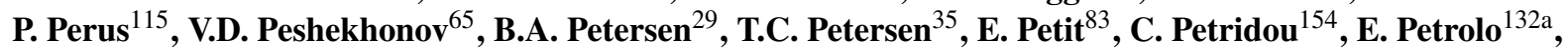
F. Petrucci ${ }^{134 a, 134 b}$, D. Petschull ${ }^{41}$, M. Petteni ${ }^{142}$, R. Pezoa ${ }^{31 b}$, A. Phan ${ }^{86}$, A.W. Phillips ${ }^{27}$, G. Piacquadio ${ }^{29}$, M. Piccinini ${ }^{19 a, 19 b}$, R. Piegaia ${ }^{26}$, J.E. Pilcher ${ }^{30}$, A.D. Pilkington ${ }^{82}$, J. Pina ${ }^{124, q}$, M. Pinamonti ${ }^{164 a, 164 c}$, J.L. Pinfold ${ }^{2}$, 
B. Pinto ${ }^{124 a, b}$, C. Pizio ${ }^{89 a, 89 b}$, R. Placakyte ${ }^{41}$, M. Plamondon ${ }^{169}$, M.-A. Pleier ${ }^{24}$, A. Poblaguev ${ }^{175}$, S. Poddar $^{58 a}$,

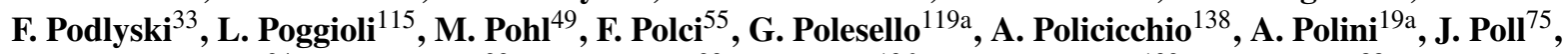
V. Polychronakos ${ }^{24}$, D. Pomeroy ${ }^{22}$, K. Pommès ${ }^{29}$, P. Ponsot ${ }^{136}$, L. Pontecorvo ${ }^{132 a}$, B.G. Pope ${ }^{88}$, G.A. Popeneciu ${ }^{25 a}$, D.S. Popovic ${ }^{12 a}$, A. Poppleton ${ }^{29}$, J. Popule ${ }^{125}$, X. Portell Bueso ${ }^{48}$, R. Porter ${ }^{163}$, G.E. Pospelov ${ }^{99}$, S. Pospisil ${ }^{127}$, M. Potekhin ${ }^{24}$, I.N. Potrap ${ }^{99}$, C.J. Potter ${ }^{149}$, C.T. Potter ${ }^{85}$, K.P. Potter ${ }^{82}$, G. Poulard ${ }^{29}$, J. Poveda ${ }^{172}$, R. Prabhu ${ }^{20}$,

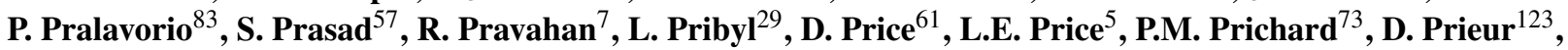
M. Primavera ${ }^{72 a}$, K. Prokofiev ${ }^{29}$, F. Prokoshin ${ }^{31 b}$, S. Protopopescu ${ }^{24}$, J. Proudfoot ${ }^{5}$, X. Prudent ${ }^{43}$, H. Przysiezniak ${ }^{4}$,

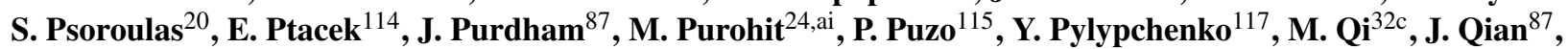

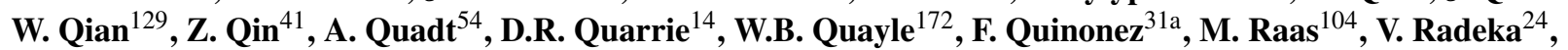
V. Radescu ${ }^{58 b}$, B. Radics $^{20}$, T. Rador ${ }^{18 a}$, F. Ragusa ${ }^{89,89 b}$, G. Rahal ${ }^{180}$, A.M. Rahimi ${ }^{109}$, S. Rajagopalan ${ }^{24}$, M. Rammensee $^{48}$, M. Rammes ${ }^{141}$, F. Rauscher ${ }^{98}$, E. Rauter ${ }^{99}$, M. Raymond ${ }^{29}$, A.L. Read $^{117}$, D.M. Rebuzzi ${ }^{119 a, 119 b}$, A. Redelbach ${ }^{173}$, G. Redlinger ${ }^{24}$, R. Reece ${ }^{120}$, K. Reeves $^{40}$, E. Reinherz-Aronis ${ }^{153}$, A. Reinsch ${ }^{114}$, I. Reisinger $^{42}$,

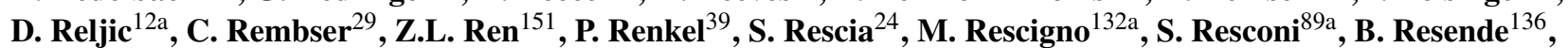
P. Reznicek ${ }^{126}$, R. Rezvani ${ }^{158}$, N. Ribeiro ${ }^{124 a}$, A. Richards ${ }^{77}$, R. Richter ${ }^{99}$, E. Richter-Was ${ }^{38, a j}$, M. Ridel ${ }^{78}$, M. Rijpstra ${ }^{105}$, M. Rijssenbeek ${ }^{148}$, A. Rimoldi ${ }^{119 a, 119 b}$, L. Rinaldi ${ }^{19 a}$, R.R. Rios $^{39}$, I. Riu ${ }^{11}$, F. Rizatdinova ${ }^{112}$, E. Rizvi ${ }^{75}$, D.A. Roa Romero ${ }^{162}$, S.H. Robertson ${ }^{85}$, , A. Robichaud-Veronneau ${ }^{49}$, D. Robinson ${ }^{27}$, J.E.M. Robinson ${ }^{77}$, M. Robinson ${ }^{114}$, A. Robson ${ }^{53}$, J.G. Rocha de Lima ${ }^{106}$, C. Roda ${ }^{122 a, 122 b}$, D. Roda Dos Santos ${ }^{29}$, D. Rodriguez ${ }^{162}$, Y. Rodriguez Garcia ${ }^{15}$, S. Roe ${ }^{29}$, O. Røhne ${ }^{117}$, V. Rojo ${ }^{1}$, S. Rolli ${ }^{161}$, A. Romaniouk ${ }^{96}$, V.M. Romanov ${ }^{65}$, G. Romeo ${ }^{26}$, D. Romero Maltrana ${ }^{31 a}$, L. $\operatorname{Roos}^{78}$, E. Ros $^{167}$, S. Rosati ${ }^{138}$, G.A. Rosenbaum ${ }^{158}$, L. Rosselet $^{49}$, V. Rossetti ${ }^{11}$, L.P. Rossi ${ }^{50 a}$, M. Rotaru ${ }^{25 a}$, J. Rothberg ${ }^{138}$, D. Rousseau ${ }^{115}$, C.R. Royon $^{136}$, A. Rozanov $^{83}$, Y. Rozen ${ }^{152}$, X. Ruan $^{115}$, B. Ruckert $^{98}$, N. Ruckstuhi ${ }^{105}$, V.I. Rud ${ }^{97}$, G. Rudolph $^{62}$, F. Rühr ${ }^{58 a}$, F. Ruggieri ${ }^{134 a}$, A. Ruiz-Martinez ${ }^{64}$, L. Rumyantsev ${ }^{65}$, Z. Rurikova ${ }^{48}$, N.A. Rusakovich ${ }^{65}$, J.P. Rutherfoord ${ }^{6}$, C. Ruwiedel $^{20}$, P. Ruzicka ${ }^{125}$, Y.F. Ryabov ${ }^{121}$, P. Ryan $^{88}$, G. Rybkin ${ }^{115}$, S. Rzaeva ${ }^{10}$, A.F. Saavedra ${ }^{150}$, H.F.-W. Sadrozinski ${ }^{137}$, R. Sadykov ${ }^{65}$, F. Safai Tehrani ${ }^{132 a, 132 b}$, H. Sakamoto ${ }^{155}$, G. Salamanna ${ }^{105}$, A. Salamon ${ }^{133 a}$, M.S. Saleem ${ }^{111}$, D. Salihagic ${ }^{99}$, A. Salnikov ${ }^{143}$, J. Salt ${ }^{167}$, B.M. Salvachua Ferrando ${ }^{5}$, D. Salvatore ${ }^{36 a, 36 b}$, F. Salvatore ${ }^{149}$, A. Salvucci ${ }^{47}$, A. Salzburger ${ }^{29}$, D. Sampsonidis ${ }^{154}$, B.H. Samset ${ }^{117}$, H. Sandaker ${ }^{13}$, H.G. Sander ${ }^{81}$, M.P. Sanders ${ }^{98}$, M. Sandhoff ${ }^{174}$, P. Sandhu ${ }^{158}$, R. Sandstroem ${ }^{105}$, S. Sandvoss ${ }^{174}$, D.P.C. Sankey ${ }^{129}$, B. Sanny ${ }^{174}$, A. Sansoni ${ }^{47}$, C. Santamarina Rios $^{85}$, C. Santoni ${ }^{33}$, R. Santonico ${ }^{133 a, 133 b}$, J.G. Saraiva ${ }^{124 a, q}$, T. Sarangi ${ }^{172}$, E. Sarkisyan-Grinbaum ${ }^{7}$, F. Sarri ${ }^{122 a, 122 b}$, O. Sasaki ${ }^{66}$, N. Sasao ${ }^{68}$, I. Satsounkevitch ${ }^{90}$, G. Sauvage ${ }^{4}$, P. Savard ${ }^{158, f}$, A.Y. Savine ${ }^{6}$, V. Savinov ${ }^{123}$, L. Sawyer ${ }^{24, a k}$, D.H. Saxon ${ }^{53}$, L.P. Says ${ }^{33}$, C. Sbarra ${ }^{19 a}$,19b, A. Sbrizzi ${ }^{19 a, 19 b}$, D.A. Scannicchio ${ }^{29}$, J. Schaarschmidt ${ }^{43}$, P. Schacht ${ }^{99}$, U. Schäfer ${ }^{81}$, S. Schaetzel ${ }^{58 b}$, A.C. Schaffer ${ }^{115}$, D. Schaile ${ }^{98}$, R.D. Schamberger ${ }^{148}$, A.G. Schamov ${ }^{107}$, V. Scharf ${ }^{58 a}$, V.A. Schegelsky ${ }^{121}$, D. Scheirich ${ }^{87}$, M. Schernau ${ }^{163}$, M.I. Scherzer ${ }^{14}$, C. Schiavi ${ }^{50,50 b}$, J. Schieck ${ }^{99}$, M. Schioppa ${ }^{36 a, 36 b}$, S. Schlenker ${ }^{29}$, E. Schmidt ${ }^{48}$, K. Schmieden ${ }^{20}$, C. Schmitt ${ }^{81}$, M. Schmitz ${ }^{20}$, A. Schönig ${ }^{58 b}$, M. Schott ${ }^{29}$, D. Schouten ${ }^{142}$, J. Schovancova ${ }^{125}$, M. Schram ${ }^{85}$, A. Schreiner ${ }^{63}$, C. Schroeder ${ }^{81}$, N. Schroer ${ }^{58 c}$, M. Schroers ${ }^{174}$, J. Schultes ${ }^{174}$, H.-C. Schultz-Coulon ${ }^{58 a}$, J.W. Schumacher ${ }^{43}$, M. Schumacher ${ }^{48}$, B.A. Schumm ${ }^{137}$, Ph. Schune ${ }^{136}$, C. Schwanenberger ${ }^{82}$, A. Schwartzman ${ }^{143}$, Ph. Schwemling ${ }^{78}$, R. Schwienhorst ${ }^{88}$, R. Schwierz ${ }^{43}$, J. Schwindling ${ }^{136}$, W.G. Scott ${ }^{129}$, J. Searcy ${ }^{114}$, E. Sedykh ${ }^{121}$, E. Segura ${ }^{11}$, S.C. Seidel ${ }^{103}$, A. Seiden ${ }^{137}$, F. Seifert ${ }^{43}$, J.M. Seixas ${ }^{23 a}$, G. Sekhniaidze ${ }^{102 a}$, D.M. Seliverstov ${ }^{121}$, B. Sellden ${ }^{146 a}$, N. Semprini-Cesari ${ }^{19 a, 19 b}$, C. Serfon ${ }^{98}$, L. Serin ${ }^{115}$, R. Seuster ${ }^{99}$, H. Severini ${ }^{111}$, M.E. Sevior ${ }^{86}$, A. Sfyrla ${ }^{165}$, E. Shabalina ${ }^{54}$, M. Shamim ${ }^{114}$, L.Y. Shan ${ }^{32 a}$, J.T. Shank ${ }^{21}$, Q.T. Shao ${ }^{86}$, M. Shapiro ${ }^{14}$, P.B. Shatalov ${ }^{95}$, K. Shaw ${ }^{139}$, D. Sherman ${ }^{29}$, P. Sherwood ${ }^{77}$, A. Shibata ${ }^{108}$, M. Shimojima ${ }^{100}$, T. Shin ${ }^{56}$, A. Shmeleva ${ }^{94}$, M.J. Shochet ${ }^{30}$, M.A. Shupe ${ }^{6}$, P. Sicho ${ }^{125}$, A. Sidoti ${ }^{15}$, F. Siegert ${ }^{77}$, J. Siegrist ${ }^{14}$, Dj. Sijacki ${ }^{12 a}$, O. Silbert ${ }^{171}$, J. Silva ${ }^{124 a, a l}$, Y. Silver ${ }^{153}$, D. Silverstein ${ }^{143}$, S.B. Silverstein ${ }^{146 a}$, V. Simak ${ }^{127}$, Lj. Simic ${ }^{12 a}$, S. Simion ${ }^{115}$, B. Simmons ${ }^{77}$, M. Simonyan ${ }^{35}$, P. Sinervo ${ }^{158}$, N.B. Sinev ${ }^{114}$, V. Sipica ${ }^{141}$, G. Siragusa $^{81}$, A.N. Sisakyan ${ }^{65}$, S.Yu. Sivoklokov ${ }^{97}$, J. Sjoelin ${ }^{146 a, 146 b}$, T.B. Sjursen ${ }^{13}$, K. Skovpen ${ }^{107}$, P. Skubic ${ }^{111}$, M. Slater ${ }^{17}$, T. Slavicek ${ }^{127}$, K. Sliwa ${ }^{161}$, J. Sloper ${ }^{29}$, V. Smakhtin ${ }^{171}$, S.Yu. Smirnov ${ }^{96}$, Y. Smirnov ${ }^{24}$, L.N. Smirnova ${ }^{97}$, O. Smirnova ${ }^{79}$, B.C. Smith ${ }^{57}$, D. Smith ${ }^{143}$, K.M. Smith ${ }^{53}$, M. Smizanska ${ }^{71}$, K. Smolek ${ }^{127}$, A.A. Snesarev ${ }^{94}$, S.W. Snow ${ }^{82}$, J. Snow ${ }^{111}$, J. Snuverink ${ }^{105}$, S. Snyder ${ }^{24}$, M. Soares ${ }^{124 a}$, R. Sobie ${ }^{169, j}$, J. Sodomka ${ }^{127}$, A. Soffer ${ }^{153}$, C.A. Solans ${ }^{167}$, M. Solar ${ }^{127}$, J. Solc ${ }^{127}$, E. Solfaroli Camillocci ${ }^{132 a, 132 b}$, A.A. Solodkov ${ }^{128}$, O.V. Solovyanov ${ }^{128}$, J. Sondericker ${ }^{24}$, V. Sopko ${ }^{127}$, B. Sopko ${ }^{127}$, M. Sosebee ${ }^{7}$, A. Soukharev ${ }^{107}$, S. Spagnolo ${ }^{72 a, 72 b}$, F. Spanò ${ }^{34}$, R. Spighi ${ }^{19 a}$, G. Spigo ${ }^{29}$, F. Spila ${ }^{132 a, 132 b}$, R. Spiwoks ${ }^{29}$, M. Spousta ${ }^{126}$, T. Spreitzer ${ }^{142}$, B. Spurlock ${ }^{7}$, R.D. St. Denis ${ }^{53}$, T. Stahl ${ }^{141}$, J. Stahlman ${ }^{120}$, R. Stamen $^{58 a}$, S.N. Stancu ${ }^{163}$, E. Stanecka ${ }^{29}$, R.W. Stanek ${ }^{5}$, C. Stanescu $^{134 a}$, S. Stapnes ${ }^{117}$, E.A. Starchenko ${ }^{128}$, J. Stark ${ }^{55}$, P. Staroba ${ }^{125}$, P. Starovoitov ${ }^{91}$, J. Stastny ${ }^{125}$, 
P. Stavina ${ }^{144 a}$, G. Steele ${ }^{53}$, P. Steinbach ${ }^{43}$, P. Steinberg ${ }^{24}$, I. Stekl ${ }^{127}$, B. Stelzer ${ }^{142}$, H.J. Stelzer ${ }^{41}$,

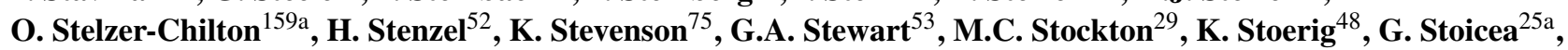
S. Stonjek ${ }^{99}$, P. Strachota ${ }^{126}$, A.R. Stradling ${ }^{7}$, A. Straessner ${ }^{43}$, J. Strandberg ${ }^{87}$, S. Strandberg ${ }^{14}$, A. Strandlie ${ }^{117}$, M. Strauss ${ }^{111}$, P. Strizenec ${ }^{144 b}$, R. Ströhmer ${ }^{173}$, D.M. Strom ${ }^{114}$, R. Stroynowski ${ }^{39}$, J. Strube $^{129}$, B. Stugu ${ }^{13}$, P. Sturm ${ }^{174}$, D.A. Soh ${ }^{151, \text { am }}$, D. Su ${ }^{143}$, Y. Sugaya ${ }^{116}$, T. Sugimoto ${ }^{101}$, C. Suhr ${ }^{106}$, M. Suk ${ }^{126}$, V.V. Sulin ${ }^{94}$, S. Sultansoy ${ }^{3 \mathrm{~d}}$, T. Sumida ${ }^{29}$, X.H. Sun ${ }^{32 \mathrm{~d}}$, J.E. Sundermann ${ }^{48}$, K. Suruliz ${ }^{164 \mathrm{a}, 164 \mathrm{~b}}$, S. Sushkov ${ }^{11}$, G. Susinno ${ }^{36 a, 36 b}$, M.R. Sutton ${ }^{139}$, T. Suzuki ${ }^{155}$, Y. Suzuki ${ }^{66}$, I. Sykora ${ }^{144 a}$, T. Sykora ${ }^{126}$, T. Szymocha ${ }^{38}$, J. Sánchez ${ }^{167}$, D. Ta ${ }^{20}$, K. Tackmann ${ }^{29}$, A. Taffard ${ }^{163}$, R. Tafirout ${ }^{159 a}$, A. Taga ${ }^{117}$, Y. Takahashi ${ }^{101}$, H. Takai ${ }^{24}$, R. Takashima ${ }^{69}$, H. Takeda ${ }^{67}$, T. Takeshita ${ }^{140}$, M. Talby ${ }^{83}$, A. Talyshev ${ }^{107}$, M.C. Tamsett ${ }^{76}$, J. Tanaka $^{155}$, R. Tanaka ${ }^{115}$, S. Tanaka $^{131}$, S. Tanaka ${ }^{66}$, S. Tapprogge ${ }^{81}$, D. Tardif ${ }^{158}$, S. Tarem ${ }^{152}$, F. Tarrade ${ }^{24}$, G.F. Tartarelli ${ }^{89 a}$, P. Tas ${ }^{126}$, M. Tasevsky ${ }^{125}$, E. Tassi ${ }^{36 a, 36 b}$, M. Tatarkhanov ${ }^{14}$, C. Taylor ${ }^{77}$, F.E. Taylor ${ }^{92}$, G.N. Taylor ${ }^{86}$, R.P. Taylor ${ }^{169}$, W. Taylor ${ }^{159 b}$,

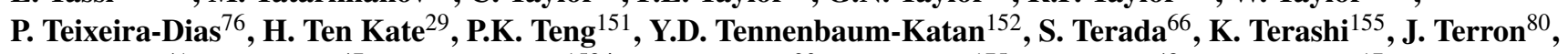
M. Terwort ${ }^{41, v}$, M. Testa ${ }^{47}$, R.J. Teuscher ${ }^{158, j}$, J. Therhaag ${ }^{20}$, M. Thioye ${ }^{175}$, S. Thoma ${ }^{48}$, J.P. Thomas ${ }^{17}$, E.N. Thompson ${ }^{84}$, P.D. Thompson ${ }^{17}$, P.D. Thompson ${ }^{158}$, R.J. Thompson ${ }^{82}$, A.S. Thompson ${ }^{53}$, E. Thomson ${ }^{120}$, R.P. Thun ${ }^{87}$, T. Tic ${ }^{125}$, V.O. Tikhomirov ${ }^{94}$, Y.A. Tikhonov ${ }^{107}$, P. Tipton ${ }^{175}$, F.J. Tique Aires Viegas ${ }^{29}$, S. Tisserant ${ }^{83}$,

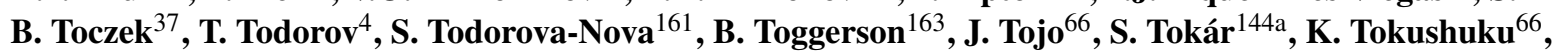
K. Tollefson ${ }^{88}$, L. Tomasek ${ }^{125}$, M. Tomasek ${ }^{125}$, M. Tomoto ${ }^{101}$, L. Tompkins ${ }^{14}$, K. Toms ${ }^{103}$, A. Tonoyan ${ }^{13}$, C. Topfel ${ }^{16}$,

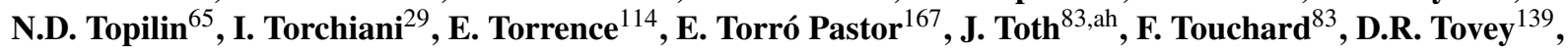

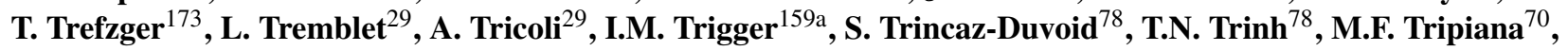

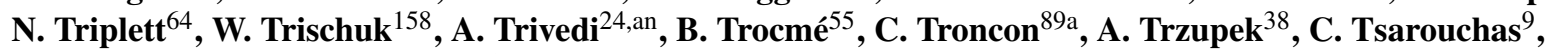
J.C.-L. Tseng ${ }^{118}$, M. Tsiakiris ${ }^{105}$, P.V. Tsiareshka ${ }^{90}$, D. Tsionou ${ }^{139}$, G. Tsipolitis $^{9}$, V. Tsiskaridze ${ }^{51}$,

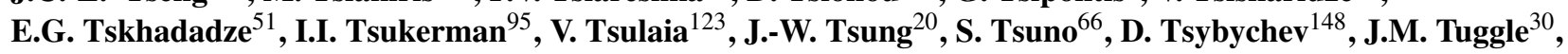
C.D. Tunnell ${ }^{30}$, D. Turecek ${ }^{127}$, I. Turk Cakir ${ }^{3 e}$, E. Turlay ${ }^{105}$, P.M. Tuts ${ }^{34}$, M.S. Twomey ${ }^{138}$, M. Tylmad ${ }^{146 a, 146 b}$, M. Tyndel ${ }^{129}$, K. Uchida ${ }^{116}$, I. Ueda ${ }^{155}$, R. Ueno ${ }^{28}$, M. Ugland ${ }^{13}$, M. Uhlenbrock ${ }^{20}$, M. Uhrmacher $^{54}$, F. Ukegawa ${ }^{160}$,

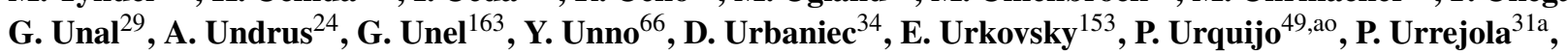

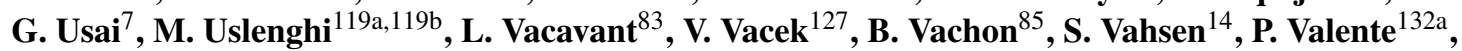
S. Valentinetti ${ }^{19 a, 19 b}$, A. Valero ${ }^{167}$, S. Valkar ${ }^{126}$, E. Valladolid Gallego ${ }^{167}$, S. Vallecorsa ${ }^{152}$, J.A. Valls Ferrer ${ }^{167}$, R. Van Berg ${ }^{120}$, H. van der Graaf ${ }^{105}$, E. van der Kraaij ${ }^{105}$, E. van der Poel ${ }^{105}$, D. van der Ster ${ }^{29}$, N. van Eldik ${ }^{84}$, P. van Gemmeren ${ }^{5}$, Z. van Kesteren ${ }^{105}$, I. van Vulpen ${ }^{105}$, W. Vandelli $^{29}$, A. Vaniachine $^{5}$, P. Vankov ${ }^{73}$, F. Vannucci $^{78}$, R. Vari ${ }^{132 a}$, E.W. Varnes $^{6}$, D. Varouchas ${ }^{14}$, A. Vartapetian $^{7}$, K.E. Varvell ${ }^{150}$, L. Vasilyeva ${ }^{94}$, V.I. Vassilakopoulos ${ }^{56}$, F. Vazeille ${ }^{33}$, C. Vellidis $^{8}$, F. Veloso ${ }^{124 a}$, S. Veneziano ${ }^{132 a}$, A. Ventura ${ }^{72 a, 72 b}$, D. Ventura ${ }^{138}$, M. Venturi $^{48}$, N. Venturi $^{16}$, V. Vercesi ${ }^{119 a}$, M. Verducci ${ }^{173}$, W. Verkerke ${ }^{105}$, J.C. Vermeulen ${ }^{105}$, M.C. Vetterli ${ }^{142, f}$, I. Vichou ${ }^{165}$, T. Vickey ${ }^{145 \text { bap, }}$ G.H.A. Viehhauser ${ }^{118}$, M. Villa ${ }^{19 a, 19 b}$, E.G. Villani ${ }^{129}$, M. Villaplana Perez ${ }^{167}$, E. Vilucchi $^{47}$, M.G. Vincter ${ }^{28}$,

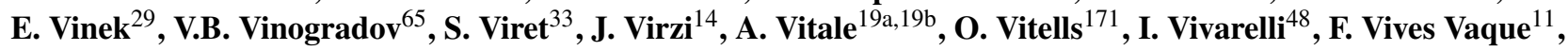
S. Vlachos ${ }^{9}$, M. Vlasak ${ }^{127}$, N. Vlasov ${ }^{20}$, A. Vogel ${ }^{20}$, P. Vokac ${ }^{127}$, M. Volpi ${ }^{11}$, H. von der Schmitt ${ }^{99}$, J. von Loeben ${ }^{99}$, H. von Radziewski ${ }^{48}$, E. von Toerne ${ }^{20}$, V. Vorobel ${ }^{126}$, V. Vorwerk $^{11}$, M. Vos ${ }^{167}$, R. Voss ${ }^{29}$, T.T. Voss ${ }^{174}$, J.H. Vossebeld ${ }^{73}$, N. Vranjes ${ }^{12 a}$, M. Vranjes Milosavljevic ${ }^{12 a}$, V. Vrba ${ }^{125}$, M. Vreeswijk ${ }^{105}$, T. Vu Anh ${ }^{81}$, D. Vudragovic $^{12 a}$, R. Vuillermet ${ }^{29}$, I. Vukotic ${ }^{115}$, P. Wagner ${ }^{120}$, J. Walbersloh ${ }^{42}$, J. Walder $^{71}$, R. Walker ${ }^{98}$, W. Walkowiak ${ }^{141}$, R. Wall ${ }^{175}$, C. Wang ${ }^{44}$, H. Wang ${ }^{172}$, J. Wang ${ }^{55}$, S.M. Wang ${ }^{151}$, A. Warburton ${ }^{85}$, C.P. Ward ${ }^{27}$, M. Warsinsky ${ }^{48}$, R. Wastie ${ }^{118}$, P.M. Watkins ${ }^{17}$, A.T. Watson ${ }^{17}$, M.F. Watson ${ }^{17}$, G. Watts ${ }^{138}$, S. Watts ${ }^{82}$, A.T. Waugh ${ }^{150}$, B.M. Waugh ${ }^{77}$, M.D. Weber ${ }^{16}$, M. Weber ${ }^{129}$, M.S. Weber ${ }^{16}$, P. Weber $^{58 a}$, A.R. Weidberg ${ }^{118}$, J. Weingarten ${ }^{54}$, C. Weiser ${ }^{48}$, H. Wellenstein ${ }^{22}$, P.S. Wells ${ }^{29}$, T. Wenaus ${ }^{24}$, S. Wendler ${ }^{123}$, Z. Weng ${ }^{151, \text { aq }}$, T. Wengler ${ }^{82}$, S. Wenig ${ }^{29}$, N. Wermes ${ }^{20}$, M. Werner ${ }^{48}$, P. Werner ${ }^{29}$, M. Werth ${ }^{163}$, U. Werthenbach ${ }^{141}$, M. Wessels ${ }^{58 a}$, K. Whalen ${ }^{28}$, A. White ${ }^{7}$, M.J. White ${ }^{27}$, S. White ${ }^{24}$, S.R. Whitehead ${ }^{118}$, D. Whiteson ${ }^{163}$, D. Whittington ${ }^{61}$, F. Wicek $^{115}$, D. Wicke ${ }^{81}$, F.J. Wickens ${ }^{129}$, W. Wiedenmann ${ }^{172}$, M. Wielers ${ }^{129}$, P. Wienemann ${ }^{20}$, C. Wiglesworth ${ }^{73}$, L.A.M. Wiik ${ }^{48}$, A. Wildauer ${ }^{167}$, M.A. Wildt ${ }^{41, v}$, H.G. Wilkens ${ }^{29}$, E. Williams ${ }^{34}$, H.H. Williams ${ }^{120}$, S. Willocq ${ }^{84}$, J.A. Wilson ${ }^{17}$, M.G. Wilson ${ }^{143}$, A. Wilson ${ }^{87}$, I. Wingerter-Seez ${ }^{4}$, F. Winklmeier ${ }^{29}$, M. Wittgen ${ }^{143}$, M.W. Wolter ${ }^{38}$, H. Wolters ${ }^{124 a, g}$, B.K. Wosiek ${ }^{38}$, J. Wotschack ${ }^{29}$, M.J. Woudstra ${ }^{84}$, K. Wraight ${ }^{53}$, C. Wright $^{53}$, D. Wright ${ }^{143}$, B. Wrona ${ }^{73}$, S.L. Wu ${ }^{172}$, X. Wu ${ }^{49}$, E. Wulf ${ }^{34}$, B.M. Wynne ${ }^{45}$, L. Xaplanteris ${ }^{9}$, S. Xella ${ }^{35}$, S. Xie ${ }^{48}$, D. Xu ${ }^{139}$, N. Xu ${ }^{172}$, M. Yamada ${ }^{160}$, A. Yamamoto ${ }^{66}$, K. Yamamoto ${ }^{64}$, S. Yamamoto ${ }^{155}$, T. Yamamura ${ }^{155}$, J. Yamaoka ${ }^{44}$, T. Yamazaki ${ }^{155}$,

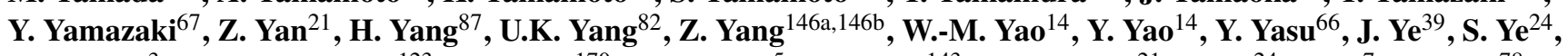

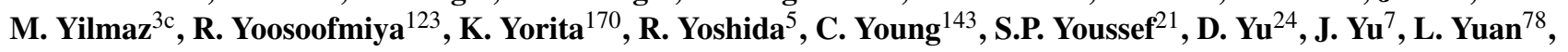


A. Yurkewicz ${ }^{148}$, R. Zaidan ${ }^{63}$, A.M. Zaitsev ${ }^{128}$, Z. Zajacova $^{29}$, V. Zambrano ${ }^{47}$, L. Zanello ${ }^{132 a, 132 b}$, A. Zaytsev ${ }^{107}$, C. Zeitnitz ${ }^{174}$, M. Zeller ${ }^{175}$, A. Zemla ${ }^{38}$, C. Zendler ${ }^{20}$, O. Zenin ${ }^{128}$, T. Zenis ${ }^{144 a}$, Z. Zenonos ${ }^{122 a, 122 b}$, S. Zenz ${ }^{14}$, D. Zerwas ${ }^{115}$, G. Zevi della Porta ${ }^{57}$, Z. Zhan ${ }^{32 \mathrm{~d}}$, H. Zhang ${ }^{83}$, J. Zhang ${ }^{5}$, Q. Zhang ${ }^{5}$, X. Zhang ${ }^{32 \mathrm{~d}}$, L. Zhao ${ }^{108}$, T. Zhao ${ }^{138}$, Z. Zhao ${ }^{32 b}$, A. Zhemchugov ${ }^{65}$, J. Zhong ${ }^{151, \text { ar }}$, B. Zhou ${ }^{87}$, N. Zhou ${ }^{34}$, Y. Zhou ${ }^{151}$, C.G. Zhu ${ }^{32 d}$, H. Zhu ${ }^{41}$, Y. Zhu ${ }^{172}$, X. Zhuang ${ }^{98}$, V. Zhuravlov ${ }^{99}$, R. Zimmermann ${ }^{20}$, S. Zimmermann ${ }^{20}$, S. Zimmermann ${ }^{48}$, M. Ziolkowski ${ }^{141}$, L. Živković ${ }^{34}$, G. Zobernig ${ }^{172}$, A. Zoccoli ${ }^{19 a, 19 b}$, M. zur Nedden ${ }^{15}$, V. Zutshi ${ }^{106}$

${ }^{\star}$ CERN, 1211 Geneva 23, Switzerland

${ }^{1}$ University at Albany, 1400 Washington Ave, Albany, NY 12222, United States of America

${ }^{2}$ University of Alberta, Department of Physics, Centre for Particle Physics, Edmonton, AB T6G 2G7, Canada

${ }^{3}$ Ankara University ${ }^{(a)}$, Faculty of Sciences, Department of Physics, TR 061000 Tandogan, Ankara; Dumlupinar University ${ }^{(b)}$, Faculty of Arts and Sciences, Department of Physics, Kutahya; Gazi University ${ }^{(\mathrm{c})}$, Faculty of Arts and Sciences, Department of Physics, 06500,

Teknikokullar, Ankara; TOBB University of Economics and Technology ${ }^{(\mathrm{d})}$, Faculty of Arts and Sciences, Division of Physics, 06560 , Sogutozu, Ankara; Turkish Atomic Energy Authority ${ }^{(\mathrm{e})}$, 06530, Lodumlu, Ankara, Turkey

${ }^{4}$ LAPP, Université de Savoie, CNRS/IN2P3, Annecy-le-Vieux, France

${ }^{5}$ Argonne National Laboratory, High Energy Physics Division, 9700 S. Cass Avenue, Argonne IL 60439, United States of America

${ }^{6}$ University of Arizona, Department of Physics, Tucson, AZ 85721, United States of America

${ }^{7}$ The University of Texas at Arlington, Department of Physics, Box 19059, Arlington, TX 76019, United States of America

${ }^{8}$ University of Athens, Nuclear \& Particle Physics, Department of Physics, Panepistimiopouli, Zografou, GR 15771 Athens, Greece

${ }^{9}$ National Technical University of Athens, Physics Department, 9-Iroon Polytechniou, GR 15780 Zografou, Greece

${ }^{10}$ Institute of Physics, Azerbaijan Academy of Sciences, H. Javid Avenue 33, AZ 143 Baku, Azerbaijan

${ }^{11}$ Institut de Física d'Altes Energies, IFAE, Edifici Cn, Universitat Autònoma de Barcelona, ES-08193 Bellaterra (Barcelona), Spain

${ }^{12}$ University of Belgrade $^{(\mathrm{a})}$, Institute of Physics, P.O. Box 57, 11001 Belgrade; Vinca Institute of Nuclear Sciences ${ }^{(\mathrm{b})}$ M. Petrovica Alasa 12-14, 11000 Belgrade, Serbia, Serbia

${ }^{13}$ University of Bergen, Department for Physics and Technology, Allegaten 55, NO-5007 Bergen, Norway

${ }^{14}$ Lawrence Berkeley National Laboratory and University of California, Physics Division, MS50B-6227, 1 Cyclotron Road, Berkeley, CA 94720, United States of America

${ }^{15}$ Humboldt University, Institute of Physics, Berlin, Newtonstr. 15, D-12489 Berlin, Germany

${ }^{16}$ University of Bern, instein Center for Fundamental Physics, ry for High Energy Physics, Sidlerstrasse 5, CH-3012 Bern, Switzerland

${ }^{17}$ University of Birmingham, School of Physics and Astronomy, Edgbaston, Birmingham B15 2TT, United Kingdom

${ }^{18}$ Bogazici University ${ }^{(a)}$, Faculty of Sciences, Department of Physics, TR-80815 Bebek-Istanbul; Dogus University ${ }^{(b)}$, Faculty of Arts and Sciences, Department of Physics, 34722, Kadikoy, Istanbul; ${ }^{(c)}$ Gaziantep University, Faculty of Engineering, Department of Physics Engineering, 27310, Sehitkamil, Gaziantep, Turkey; Istanbul Technical University (d) ${ }^{(2}$ Faculty of Arts and Sciences, Department of Physics, 34469, Maslak, Istanbul, Turkey

${ }^{19}$ INFN Sezione di Bologna ${ }^{(a)}$; Università di Bologna, Dipartimento di Fisica ${ }^{(\mathrm{b})}$, viale C. Berti Pichat, 6/2, IT-40127 Bologna, Italy

${ }^{20}$ University of Bonn, Physikalisches Institut, Nussallee 12, D-53115 Bonn, Germany

${ }^{21}$ Boston University, Department of Physics, 590 Commonwealth Avenue, Boston, MA 02215, United States of America

${ }^{22}$ Brandeis University, Department of Physics, MS057, 415 South Street, Waltham, MA 02454, United States of America

${ }^{23}$ Universidade Federal do Rio De Janeiro, COPPE/EE/IF ${ }^{\text {(a) }}$, Caixa Postal 68528, Ilha do Fundao, BR-21945-970 Rio de Janeiro;

(b) Universidade de Sao Paulo, Instituto de Fisica, R.do Matao Trav. R.187, Sao Paulo-SP, 05508-900, Brazil

${ }^{24}$ Brookhaven National Laboratory, Physics Department, Bldg. 510A, Upton, NY 11973, United States of America

${ }^{25}$ National Institute of Physics and Nuclear Engineering ${ }^{(a)}$, Bucharest-Magurele, Str. Atomistilor 407, P.O. Box MG-6, R-077125, Romania; University Politehnica Bucharest $^{(\mathrm{b})}$, Rectorat-AN 001, 313 Splaiul Independentei, sector 6, 060042 Bucuresti; West University ${ }^{(\mathrm{c})}$ in Timisoara, Bd. Vasile Parvan 4, Timisoara, Romania

${ }^{26}$ Universidad de Buenos Aires, FCEyN, Dto. Fisica, Pab I-C. Universitaria, 1428 Buenos Aires, Argentina

${ }^{27}$ University of Cambridge, Cavendish Laboratory, J J Thomson Avenue, Cambridge CB3 OHE, United Kingdom

${ }^{28}$ Carleton University, Department of Physics, 1125 Colonel By Drive, Ottawa ON K1S 5B6, Canada

${ }^{29} \mathrm{CERN}, \mathrm{CH}-1211$ Geneva 23, Switzerland

${ }^{30}$ University of Chicago, Enrico Fermi Institute, 5640 S. Ellis Avenue, Chicago, IL 60637, United States of America

${ }^{31}$ Pontificia Universidad Católica de Chile, Facultad de Fisica, Departamento de Fisica ${ }^{(a)}$, Avda. Vicuna Mackenna 4860, San Joaquin, Santiago; Universidad Técnica Federico Santa María, Departamento de Física ${ }^{(b)}$, Avda. Espãna 1680, Casilla 110-V, Valparaíso, Chile

${ }^{32}$ Institute of High Energy Physics, Chinese Academy of Sciences ${ }^{(a)}$, P.O. Box 918, 19 Yuquan Road, Shijing Shan District, CN-Beijing 100049; University of Science \& Technology of China (USTC), Department of Modern Physics ${ }^{(b)}$, Hefei, CN-Anhui 230026; Nanjing University, Department of Physics ${ }^{(\mathrm{c})}, 22$ Hankou Road, Nanjing, 210093; Shandong University, High Energy Physics Group ${ }^{(\mathrm{d})}$, Jinan, CN-Shandong 250100, China

${ }^{33}$ Laboratoire de Physique Corpusculaire, Clermont Université, Université Blaise Pascal, CNRS/IN2P3, FR-63177 Aubiere Cedex, France

${ }^{34}$ Columbia University, Nevis Laboratory, 136 So. Broadway, Irvington, NY 10533, United States of America

${ }^{35}$ University of Copenhagen, Niels Bohr Institute, Blegdamsvej 17, DK-2100 Kobenhavn 0, Denmark

${ }^{36}$ INFN Gruppo Collegato di Cosenza ${ }^{(a)}$; Università della Calabria, Dipartimento di Fisica ${ }^{(b)}$, IT-87036 Arcavacata di Rende, Italy

${ }^{37}$ Faculty of Physics and Applied Computer Science of the AGH-University of Science and Technology, (FPACS, AGH-UST), al. Mickiewicza 30, PL-30059 Cracow, Poland

${ }^{38}$ The Henryk Niewodniczanski Institute of Nuclear Physics, Polish Academy of Sciences, ul. Radzikowskiego 152, PL-31342 Krakow, Poland

${ }^{39}$ Southern Methodist University, Physics Department, 106 Fondren Science Building, Dallas, TX 75275-0175, United States of America

${ }^{40}$ University of Texas at Dallas, 800 West Campbell Road, Richardson, TX 75080-3021, United States of America

${ }^{41}$ DESY, Notkestr. 85, D-22603 Hamburg and Platanenallee 6, D-15738 Zeuthen, Germany 
${ }^{42}$ TU Dortmund, Experimentelle Physik IV, DE-44221 Dortmund, Germany

${ }^{43}$ Technical University Dresden, Institut für Kern- und Teilchenphysik, Zellescher Weg 19, D-01069 Dresden, Germany

${ }^{44}$ Duke University, Department of Physics, Durham, NC 27708, United States of America

${ }^{45}$ University of Edinburgh, School of Physics \& Astronomy, James Clerk Maxwell Building, The Kings Buildings, Mayfield Road, Edinburgh EH9 3JZ, United Kingdom

${ }^{46}$ Fachhochschule Wiener Neustadt; Johannes Gutenbergstrasse 3 AT-2700 Wiener Neustadt, Austria

${ }^{47}$ INFN Laboratori Nazionali di Frascati, via Enrico Fermi 40, IT-00044 Frascati, Italy

${ }^{48}$ Albert-Ludwigs-Universität, Fakultät für Mathematik und Physik, Hermann-Herder Str. 3, D-79104 Freiburg i.Br., Germany

${ }^{49}$ Université de Genève, Section de Physique, 24 rue Ernest Ansermet, CH-1211 Geneve 4, Switzerland

${ }^{50}$ INFN Sezione di Genova $^{(a)}$; Università di Genova, Dipartimento di Fisica ${ }^{(b)}$, via Dodecaneso 33, IT-16146 Genova, Italy

${ }^{51}$ Institute of Physics of the Georgian Academy of Sciences, 6 Tamarashvili St., GE-380077 Tbilisi; Tbilisi State University, HEP Institute, University St. 9, GE-380086 Tbilisi, Georgia

${ }^{52}$ Justus-Liebig-Universität Giessen, II Physikalisches Institut, Heinrich-Buff Ring 16, D-35392 Giessen, Germany

${ }^{53}$ University of Glasgow, Department of Physics and Astronomy, Glasgow G12 8QQ, United Kingdom

${ }^{54}$ Georg-August-Universität, II. Physikalisches Institut, Friedrich-Hund Platz 1, D-37077 Göttingen, Germany

${ }^{55}$ Laboratoire de Physique Subatomique et de Cosmologie, CNRS/IN2P3, Université Joseph Fourier, INPG, 53 avenue des Martyrs, FR-38026 Grenoble Cedex, France

${ }^{56}$ Hampton University, Department of Physics, Hampton, VA 23668, United States of America

${ }^{57}$ Harvard University, Laboratory for Particle Physics and Cosmology, 18 Hammond Street, Cambridge, MA 02138, United States of America

${ }^{58}$ Ruprecht-Karls-Universität Heidelberg: Kirchhoff-Institut für Physik ${ }^{(a)}$, Im Neuenheimer Feld 227, D-69120 Heidelberg; Physikalisches Institut $^{(\mathrm{b})}$, Philosophenweg 12, D-69120 Heidelberg; ZITI Ruprecht-Karls-University Heidelberg ${ }^{(\mathrm{c})}$, Lehrstuhl für Informatik V, B6, 23-29, DE-68131 Mannheim, Germany

${ }^{59}$ Hiroshima University, Faculty of Science, 1-3-1 Kagamiyama, Higashihiroshima-shi, JP-Hiroshima 739-8526, Japan

${ }^{60}$ Hiroshima Institute of Technology, Faculty of Applied Information Science, 2-1-1 Miyake Saeki-ku, Hiroshima-shi, JP-Hiroshima 731-5193, Japan

${ }^{61}$ Indiana University, Department of Physics, Swain Hall West 117, Bloomington, IN 47405-7105, United States of America

${ }^{62}$ Institut für Astro- und Teilchenphysik, Technikerstrasse 25, A-6020 Innsbruck, Austria

${ }^{63}$ University of Iowa, 203 Van Allen Hall, Iowa City, IA 52242-1479, United States of America

${ }^{64}$ Iowa State University, Department of Physics and Astronomy, Ames High Energy Physics Group, Ames, IA 50011-3160, United States of America

${ }^{65}$ Joint Institute for Nuclear Research, JINR Dubna, RU-141 980 Moscow Region, Russia

${ }^{66}$ KEK, High Energy Accelerator Research Organization, 1-1 Oho, Tsukuba-shi, Ibaraki-ken 305-0801, Japan

${ }^{67}$ Kobe University, Graduate School of Science, 1-1 Rokkodai-cho, Nada-ku, JP Kobe 657-8501, Japan

${ }^{68}$ Kyoto University, Faculty of Science, Oiwake-cho, Kitashirakawa, Sakyou-ku, Kyoto-shi, JP-Kyoto 606-8502, Japan

${ }^{69}$ Kyoto University of Education, 1 Fukakusa, Fujimori, fushimi-ku, Kyoto-shi, JP-Kyoto 612-8522, Japan

${ }^{70}$ Universidad Nacional de La Plata, FCE, Departamento de Física, IFLP (CONICET-UNLP), C.C. 67, 1900 La Plata, Argentina

${ }^{71}$ Lancaster University, Physics Department, Lancaster LA1 4YB, United Kingdom

${ }^{72}$ INFN Sezione di Lecce ${ }^{(\mathrm{a})}$; Università del Salento, Dipartimento di Fisica ${ }^{(\mathrm{b})}$ Via Arnesano IT-73100 Lecce, Italy

${ }^{73}$ University of Liverpool, Oliver Lodge Laboratory, P.O. Box 147, Oxford Street, Liverpool L69 3BX, United Kingdom

${ }^{74}$ Jožef Stefan Institute and University of Ljubljana, Department of Physics, SI-1000 Ljubljana, Slovenia

${ }^{75}$ Queen Mary University of London, Department of Physics, Mile End Road, London E1 4NS, United Kingdom

${ }^{76}$ Royal Holloway, University of London, Department of Physics, Egham Hill, Egham, Surrey TW20 0EX, United Kingdom

${ }^{77}$ University College London, Department of Physics and Astronomy, Gower Street, London WC1E 6BT, United Kingdom

${ }^{78}$ Laboratoire de Physique Nucléaire et de Hautes Energies, Université Pierre et Marie Curie (Paris 6), Université Denis Diderot (Paris-7), CNRS/IN2P3, Tour 33, 4 place Jussieu, FR-75252 Paris Cedex 05, France

${ }^{79}$ Lunds universitet, Naturvetenskapliga fakulteten, Fysiska institutionen, Box 118, SE-221 00 Lund, Sweden

${ }^{80}$ Universidad Autonoma de Madrid, Facultad de Ciencias, Departamento de Fisica Teorica, ES-28049 Madrid, Spain

${ }^{81}$ Universität Mainz, Institut für Physik, Staudinger Weg 7, DE-55099 Mainz, Germany

${ }^{82}$ University of Manchester, School of Physics and Astronomy, Manchester M13 9PL, United Kingdom

${ }^{83}$ CPPM, Aix-Marseille Université, CNRS/IN2P3, Marseille, France

${ }^{84}$ University of Massachusetts, Department of Physics, 710 North Pleasant Street, Amherst, MA 01003, United States of America

${ }^{85}$ McGill University, High Energy Physics Group, 3600 University Street, Montreal, Quebec H3A 2T8, Canada

${ }^{86}$ University of Melbourne, School of Physics, AU-Parkville, Victoria 3010, Australia

${ }^{87}$ The University of Michigan, Department of Physics, 2477 Randall Laboratory, 500 East University, Ann Arbor, MI 48109-1120, United States of America

${ }^{88}$ Michigan State University, Department of Physics and Astronomy, High Energy Physics Group, East Lansing, MI 48824-2320, United States of America

${ }^{89}$ INFN Sezione di Milano $^{(\mathrm{a})}$; Università di Milano, Dipartimento di Fisica ${ }^{(\mathrm{b})}$, via Celoria 16, IT-20133 Milano, Italy

${ }^{90}$ B.I. Stepanov Institute of Physics, National Academy of Sciences of Belarus, Independence Avenue 68, Minsk 220072, Republic of Belarus

${ }^{91}$ National Scientific \& Educational Centre for Particle \& High Energy Physics, NC PHEP BSU, M. Bogdanovich St. 153, Minsk 220040, Republic of Belarus

${ }^{92}$ Massachusetts Institute of Technology, Department of Physics, Room 24-516, Cambridge, MA 02139, United States of America

${ }^{93}$ University of Montreal, Group of Particle Physics, C.P. 6128, Succursale Centre-Ville, Montreal, Quebec, H3C 3J7, Canada

${ }^{94}$ P.N. Lebedev Institute of Physics, Academy of Sciences, Leninsky pr. 53, RU-117 924 Moscow, Russia

${ }^{95}$ Institute for Theoretical and Experimental Physics (ITEP), B. Cheremushkinskaya ul. 25, RU 117218 Moscow, Russia 
${ }^{96}$ Moscow Engineering \& Physics Institute (MEPhI), Kashirskoe Shosse 31, RU-115409 Moscow, Russia

${ }^{97}$ Lomonosov Moscow State University Skobeltsyn Institute of Nuclear Physics (MSU SINP), 1(2), Leninskie gory, GSP-1, Moscow 119991 Russian Federation, Russia

${ }^{98}$ Ludwig-Maximilians-Universität München, Fakultät für Physik, Am Coulombwall 1, DE-85748 Garching, Germany

${ }^{99}$ Max-Planck-Institut für Physik, (Werner-Heisenberg-Institut), Föhringer Ring 6, 80805 München, Germany

${ }^{100}$ Nagasaki Institute of Applied Science, 536 Aba-machi, JP Nagasaki 851-0193, Japan

${ }^{101}$ Nagoya University, Graduate School of Science, Furo-Cho, Chikusa-ku, Nagoya, 464-8602, Japan

${ }^{102}$ INFN Sezione di Napoli ${ }^{(a)}$; Università di Napoli, Dipartimento di Scienze Fisiche ${ }^{(b)}$, Complesso Universitario di Monte Sant'Angelo, via Cinthia, IT-80126 Napoli, Italy

${ }^{103}$ University of New Mexico, Department of Physics and Astronomy, MSC07 4220, Albuquerque, NM 87131 USA, United States of America

${ }^{104}$ Radboud University Nijmegen/NIKHEF, Department of Experimental High Energy Physics, Heyendaalseweg 135, NL-6525 AJ, Nijmegen, Netherlands

${ }^{105}$ Nikhef National Institute for Subatomic Physics, and University of Amsterdam, Science Park 105, 1098 XG Amsterdam, Netherlands

${ }^{106}$ Department of Physics, Northern Illinois University, LaTourette Hall ad, DeKalb, IL 60115, United States of America

${ }^{107}$ Budker Institute of Nuclear Physics (BINP), RU-Novosibirsk 630 090, Russia

${ }^{108}$ New York University, Department of Physics, 4 Washington Place, New York, NY 10003, USA

${ }^{109}$ Ohio State University, 191 West Woodruff Ave, Columbus, OH 43210-1117, United States of America

${ }^{110}$ Okayama University, Faculty of Science, Tsushimanaka 3-1-1, Okayama 700-8530, Japan

${ }^{111}$ University of Oklahoma, Homer L. Dodge Department of Physics and Astronomy, 440 West Brooks, Room 100, Norman, OK 73019-0225, United States of America

${ }^{112}$ Oklahoma State University, Department of Physics, 145 Physical Sciences Building, Stillwater, OK 74078-3072, United States of America

${ }^{113}$ Palacký University, 17.listopadu 50a, 77207 Olomouc, Czech Republic

${ }^{114}$ University of Oregon, Center for High Energy Physics, Eugene, OR 97403-1274, United States of America

${ }^{115}$ LAL, Univ. Paris-Sud, IN2P3/CNRS, Orsay, France

${ }^{116}$ Osaka University, Graduate School of Science, Machikaneyama-machi 1-1, Toyonaka, Osaka 560-0043, Japan

${ }^{117}$ University of Oslo, Department of Physics, P.O. Box 1048, Blindern, NO-0316 Oslo 3, Norway

${ }^{118}$ Oxford University, Department of Physics, Denys Wilkinson Building, Keble Road, Oxford OX1 3RH, United Kingdom

${ }^{119}$ INFN Sezione di Pavia ${ }^{(a)}$; Università di Pavia, Dipartimento di Fisica Nucleare e Teorica ${ }^{(\mathrm{b})}$, Via Bassi 6, IT-27100 Pavia, Italy

${ }^{120}$ University of Pennsylvania, Department of Physics, High Energy Physics Group, 209 S. 33rd Street, Philadelphia, PA 19104, United States of America

${ }^{121}$ Petersburg Nuclear Physics Institute, RU-188 300 Gatchina, Russia

${ }^{122}$ INFN Sezione di Pisa ${ }^{(a)}$; Università di Pisa, Dipartimento di Fisica E. Fermi ${ }^{(b)}$, Largo B. Pontecorvo 3, IT-56127 Pisa, Italy

${ }^{123}$ University of Pittsburgh, Department of Physics and Astronomy, 3941 O'Hara Street, Pittsburgh, PA 15260, United States of America

${ }^{124}$ Laboratorio de Instrumentacao e Fisica Experimental de Particulas-LIP(a), Avenida Elias Garcia 14-1, PT-1000-149 Lisboa, Portugal; Universidad de Granada, Departamento de Fisica Teorica y del Cosmos and CAFPE ${ }^{(b)}$, E-18071 Granada, Spain

${ }^{125}$ Institute of Physics, Academy of Sciences of the Czech Republic, Na Slovance 2, CZ-18221 Praha 8, Czech Republic

${ }^{126}$ Charles University in Prague, Faculty of Mathematics and Physics, Institute of Particle and Nuclear Physics, V Holesovickach 2, CZ-18000 Praha 8, Czech Republic

${ }^{127}$ Czech Technical University in Prague, Zikova 4, CZ-166 35 Praha 6, Czech Republic

${ }^{128}$ State Research Center Institute for High Energy Physics, Moscow Region, 142281, Protvino, Pobeda street, 1, Russia

${ }^{129}$ Rutherford Appleton Laboratory, Science and Technology Facilities Council, Harwell Science and Innovation Campus, Didcot OX11 0QX, United Kingdom

${ }^{130}$ University of Regina, Physics Department, Canada

${ }^{131}$ Ritsumeikan University, Noji Higashi 1 chome 1-1, JP-Kusatsu, Shiga 525-8577, Japan

${ }^{132}$ INFN Sezione di Roma I ${ }^{(a)}$; Università La Sapienza, Dipartimento di Fisica ${ }^{(b)}$, Piazzale A. Moro 2, IT- 00185 Roma, Italy

${ }^{133}$ INFN Sezione di Roma Tor Vergata $^{(a)}$; Università di Roma Tor Vergata, Dipartimento di Fisica ${ }^{(b)}$, via della Ricerca Scientifica, IT-00133 Roma, Italy

${ }^{134}$ INFN Sezione di Roma Tre ${ }^{(a)}$; Università Roma Tre, Dipartimento di Fisica ${ }^{(b)}$, via della Vasca Navale 84, IT-00146 Roma, Italy

${ }^{135}$ Réseau Universitaire de Physique des Hautes Energies (RUPHE): Université Hassan II, Faculté des Sciences Ain Chock ${ }^{(\mathrm{a})}$, B.P. 5366, MA-Casablanca; Centre National de l'Energie des Sciences Techniques Nucleaires (CNESTEN) ${ }^{(\text {b) }}$, B.P. 1382 R.P. 10001 Rabat 10001; Université Mohamed Premier ${ }^{(\mathrm{c})}$, LPTPM, Faculté des Sciences, B.P.717. Bd. Mohamed VI, 60000, Oujda; Université Mohammed V, Faculté des Sciences $^{(\mathrm{d})} 4$ Avenue Ibn Battouta, BP 1014 RP, 10000 Rabat, Morocco

${ }^{136}$ CEA, DSM/IRFU, Centre d'Etudes de Saclay, FR-91191 Gif-sur-Yvette, France

${ }^{137}$ University of California Santa Cruz, Santa Cruz Institute for Particle Physics (SCIPP), Santa Cruz, CA 95064, United States of America

${ }^{138}$ University of Washington, Seattle, Department of Physics, Box 351560, Seattle, WA 98195-1560, United States of America

${ }^{139}$ University of Sheffield, Department of Physics \& Astronomy, Hounsfield Road, Sheffield S3 7RH, United Kingdom

${ }^{140}$ Shinshu University, Department of Physics, Faculty of Science, 3-1-1 Asahi, Matsumoto-shi, JP-Nagano 390-8621, Japan

${ }^{141}$ Universität Siegen, Fachbereich Physik, D 57068 Siegen, Germany

${ }^{142}$ Simon Fraser University, Department of Physics, 8888 University Drive, CA-Burnaby, BC V5A 1S6, Canada

${ }^{143}$ SLAC National Accelerator Laboratory, Stanford, California 94309, United States of America

${ }^{144}$ Comenius University, Faculty of Mathematics, Physics \& Informatics (a) , Mlynska dolina F2, SK-84248 Bratislava; Institute of Experimental Physics of the Slovak Academy of Sciences, Dept. of Subnuclear Physics ${ }^{(b)}$, Watsonova 47, SK-04353 Kosice, Slovak Republic

145 (a) University of Johannesburg, Department of Physics, PO Box 524, Auckland Park, Johannesburg 2006; ${ }^{(b)}$ School of Physics, University of the Witwatersrand, Private Bag 3, Wits 2050, Johannesburg, South Africa, South Africa

${ }^{146}$ Stockholm University: Department of Physics ${ }^{(a)}$; The Oskar Klein Centre ${ }^{(b)}$, AlbaNova, SE-106 91 Stockholm, Sweden 
${ }^{147}$ Royal Institute of Technology (KTH), Physics Department, SE-106 91 Stockholm, Sweden

${ }^{148}$ Stony Brook University, Department of Physics and Astronomy, Nicolls Road, Stony Brook, NY 11794-3800, United States of America

${ }^{149}$ University of Sussex, Department of Physics and Astronomy 2 Building, Falmer, Brighton BN1 9QH, United Kingdom

${ }^{150}$ University of Sydney, School of Physics, AU-Sydney NSW 2006, Australia

${ }^{151}$ Insitute of Physics, Academia Sinica, TW-Taipei 11529, Taiwan

${ }^{152}$ Technion, Israel Inst. of Technology, Department of Physics, Technion City, IL-Haifa 32000, Israel

${ }^{153}$ Tel Aviv University, Raymond and Beverly Sackler School of Physics and Astronomy, Ramat Aviv, IL-Tel Aviv 69978, Israel

${ }^{154}$ Aristotle University of Thessaloniki, Faculty of Science, Department of Physics, Division of Nuclear \& Particle Physics, University Campus, GR-54124, Thessaloniki, Greece

${ }^{155}$ The University of Tokyo, International Center for Elementary Particle Physics and Department of Physics, 7-3-1 Hongo, Bunkyo-ku, JP-Tokyo 113-0033, Japan

${ }^{156}$ Tokyo Metropolitan University, Graduate School of Science and Technology, 1-1 Minami-Osawa, Hachioji, Tokyo 192-0397, Japan

${ }^{157}$ Tokyo Institute of Technology, 2-12-1-H-34 O-Okayama, Meguro, Tokyo 152-8551, Japan

${ }^{158}$ University of Toronto, Department of Physics, 60 Saint George Street, Toronto M5S 1A7, Ontario, Canada

${ }^{159}$ TRIUMF $^{(a)}$, 4004 Wesbrook Mall, Vancouver, B.C. V6T 2A3; (b) York University, Department of Physics and Astronomy, 4700 Keele St., Toronto, Ontario, M3J 1P3, Canada

${ }^{160}$ University of Tsukuba, Institute of Pure and Applied Sciences, 1-1-1 Tennoudai, Tsukuba-shi, JP-Ibaraki 305-8571, Japan

${ }^{161}$ Tufts University, Science \& Technology Center, 4 Colby Street, Medford, MA 02155, United States of America

${ }^{162}$ Universidad Antonio Narino, Centro de Investigaciones, Cra 3 Este No. 47A-15, Bogota, Colombia

${ }^{163}$ University of California, Irvine, Department of Physics \& Astronomy, CA 92697-4575, United States of America

${ }^{164}$ INFN Gruppo Collegato di Udine ${ }^{(\mathrm{a})}$; ICTP $^{(\mathrm{b})}$, Strada Costiera 11, IT-34014, Trieste; Università di Udine, Dipartimento di Fisica ${ }^{(\mathrm{c})}$, via delle Scienze 208, IT-33100 Udine, Italy

${ }^{165}$ University of Illinois, Department of Physics, 1110 West Green Street, Urbana, Illinois 61801, United States of America

${ }^{166}$ University of Uppsala, Department of Physics and Astronomy, P.O. Box 516, SE-751 20 Uppsala, Sweden

${ }^{167}$ Instituto de Física Corpuscular (IFIC) Centro Mixto UVEG-CSIC, Apdo. 22085 ES-46071 Valencia, Dept. Física At. Mol. y Nuclear; Dept. Ing. Electrónica; Univ. of Valencia, and Inst. de Microelectrónica de Barcelona (IMB-CNM-CSIC) 08193 Bellaterra, Spain

${ }^{168}$ University of British Columbia, Department of Physics, 6224 Agricultural Road, CA-Vancouver, B.C. V6T 1Z1, Canada

${ }^{169}$ University of Victoria, Department of Physics and Astronomy, P.O. Box 3055, Victoria B.C., V8W 3P6, Canada

${ }^{170}$ Waseda University, WISE, 3-4-1 Okubo, Shinjuku-ku, Tokyo, 169-8555, Japan

${ }^{171}$ The Weizmann Institute of Science, Department of Particle Physics, P.O. Box 26, IL-76100 Rehovot, Israel

${ }^{172}$ University of Wisconsin, Department of Physics, 1150 University Avenue, WI 53706 Madison, Wisconsin, United States of America

173 Julius-Maximilians-University of Würzburg, Physikalisches Institute, Am Hubland, 97074 Würzburg, Germany

${ }^{174}$ Bergische Universität, Fachbereich C, Physik, Postfach 100127, Gauss-Strasse 20, D-42097 Wuppertal, Germany

${ }^{175}$ Yale University, Department of Physics, PO Box 208121, New Haven CT, 06520-8121, United States of America

${ }^{176}$ Yerevan Physics Institute, Alikhanian Brothers Street 2, AM-375036 Yerevan, Armenia

${ }^{177}$ ATLAS-Canada Tier-1 Data Centre, TRIUMF, 4004 Wesbrook Mall, Vancouver, BC, V6T 2A3, Canada

${ }^{178}$ GridKA Tier-1 FZK, Forschungszentrum Karlsruhe GmbH, Steinbuch Centre for Computing (SCC), Hermann-von-Helmholtz-Platz 1, 76344 Eggenstein-Leopoldshafen, Germany

${ }^{179}$ Port d'Informacio Cientifica (PIC), Universitat Autonoma de Barcelona (UAB), Edifici D, E-08193 Bellaterra, Spain

${ }^{180}$ Centre de Calcul CNRS/IN2P3, Domaine scientifique de la Doua, 27 bd du 11 Novembre 1918, 69622 Villeurbanne Cedex, France

${ }^{181}$ INFN-CNAF, Viale Berti Pichat 6/2, 40127 Bologna, Italy

${ }^{182}$ Nordic Data Grid Facility, NORDUnet A/S, Kastruplundgade 22, 1, DK-2770 Kastrup, Denmark

${ }^{183}$ SARA Reken- en Netwerkdiensten, Science Park 121, 1098 XG Amsterdam, Netherlands

${ }^{184}$ Academia Sinica Grid Computing, Institute of Physics, Academia Sinica, No. 128, Sec. 2, Academia Rd., Nankang, Taipei, Taiwan 11529 , Taiwan

${ }^{185}$ UK-T1-RAL Tier-1, Rutherford Appleton Laboratory, Science and Technology Facilities Council, Harwell Science and Innovation Campus, Didcot OX11 0QX, United Kingdom

${ }^{186}$ RHIC and ATLAS Computing Facility, Physics Department, Building 510, Brookhaven National Laboratory, Upton, New York 11973, United States of America

a Also at LIP, Portugal.

${ }^{\mathrm{b}}$ Also at Faculdade de Ciencias, Universidade de Lisboa, Portugal.

${ }^{\mathrm{c}}$ Also at CPPM, Marseille, France.

${ }^{\mathrm{d}}$ Also at TRIUMF, Vancouver, Canada.

eAlso at FPACS, AGH-UST, Cracow, Poland.

${ }^{\mathrm{f}}$ Also at TRIUMF, Vancouver, Canada.

${ }^{g}$ Also at Department of Physics, University of Coimbra, Portugal.

how at CERN.

${ }^{\mathrm{i}}$ Also at Università di Napoli Parthenope, Napoli, Italy.

${ }^{\mathrm{j}}$ Also at Institute of Particle Physics (IPP), Canada.

${ }^{k}$ Also at Università di Napoli Parthenope, via A. Acton 38, IT-80133 Napoli, Italy.

${ }^{1}$ Louisiana Tech University, 305 Wisteria Street, P.O. Box 3178, Ruston, LA 71272, United States of America.

${ }^{\mathrm{m}}$ Also at Universidade de Lisboa, Portugal.

${ }^{\mathrm{n}}$ At California State University, Fresno, USA.

${ }^{\circ}$ Also at TRIUMF, 4004 Wesbrook Mall, Vancouver, B.C. V6T 2A3, Canada.

${ }^{\mathrm{p}}$ Currently at Istituto Universitario di Studi Superiori IUSS, Pavia, Italy. 
${ }^{\mathrm{q}}$ Also at Faculdade de Ciencias, Universidade de Lisboa, Portugal and at Centro de Fisica Nuclear da Universidade de Lisboa, Portugal.

${ }^{\mathrm{r}}$ Also at FPACS, AGH-UST, Cracow, Poland.

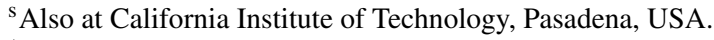

${ }^{\mathrm{t}}$ Louisiana Tech University, Ruston, USA.

uAlso at University of Montreal, Montreal, Canada.

${ }^{v}$ Also at Institut für Experimentalphysik, Universität Hamburg, Hamburg, Germany.

${ }^{\text {w}}$ Also at Petersburg Nuclear Physics Institute, Gatchina, Russia.

${ }^{x}$ Also at Institut für Experimentalphysik, Universität Hamburg, Luruper Chaussee 149, 22761 Hamburg, Germany.

${ }^{\mathrm{y}}$ Also at School of Physics and Engineering, Sun Yat-sen University, China.

${ }^{\mathrm{z}}$ Also at School of Physics, Shandong University, Jinan, China.

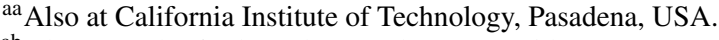

${ }^{\mathrm{ab}}$ Also at Rutherford Appleton Laboratory, Didcot, UK.

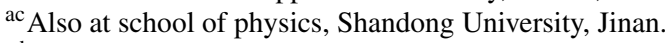

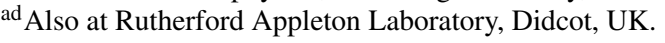

${ }^{\text {ae Now at KEK. }}$

${ }^{a f}$ Also at Departamento de Fisica, Universidade de Minho, Portugal.

${ }^{a g}$ University of South Carolina, Columbia, USA.

${ }^{\text {ah }}$ Also at KFKI Research Institute for Particle and Nuclear Physics, Budapest, Hungary.

${ }^{a i}$ University of South Carolina, Dept. of Physics and Astronomy, 700 S. Main St, Columbia, SC 29208, United States of America.

${ }^{\text {aj }}$ Also at Institute of Physics, Jagiellonian University, Cracow, Poland.

${ }^{\mathrm{ak}}$ Louisiana Tech University, Ruston, USA.

${ }^{\text {al }}$ Also at Centro de Fisica Nuclear da Universidade de Lisboa, Portugal.

${ }^{a m}$ Also at School of Physics and Engineering, Sun Yat-sen University, Taiwan.

${ }^{a n}$ University of South Carolina, Columbia, USA.

${ }^{\text {ao }}$ Transfer to LHCb 31.01.2010.

ap Also at Department of Physics, Oxford University, Oxford, United Kingdom.

${ }^{a q}$ Also at Sun Yat-sen University, Guangzhou, PR China.

${ }^{a r}$ Also at Nanjing University, China.

*Deceased

Received: 30 July 2010 / Revised: 18 October 2010 / Published online: 8 December 2010

(C) CERN for the benefit of the ATLAS collaboration 2010. This article is published with open access at Springerlink.com

\begin{abstract}
The Tile hadronic calorimeter of the ATLAS detector has undergone extensive testing in the experimental hall since its installation in late 2005. The readout, control and calibration systems have been fully operational since 2007 and the detector has successfully collected data from the LHC single beams in 2008 and first collisions in 2009. This paper gives an overview of the Tile Calorimeter performance as measured using random triggers, calibration data, data from cosmic ray muons and single beam data. The detector operation status, noise characteristics and performance of the calibration systems are presented, as well as the validation of the timing and energy calibration carried out with minimum ionising cosmic ray muons data. The calibration systems' precision is well below the design value of $1 \%$. The determination of the global energy scale was performed with an uncertainty of $4 \%$.
\end{abstract}

\section{Introduction}

The ATLAS Tile Calorimeter (TileCal) [1] is the barrel hadronic calorimeter of the ATLAS experiment [2] at the

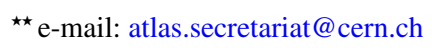

CERN Large Hadron Collider [3]. Calorimeters have a primary role in a general-purpose hadron collider detector. The ATLAS calorimeter system provides accurate energy and position measurements of electrons, photons, isolated hadrons, taus and jets. It also contributes in particle identification and in muon momentum reconstruction. In the barrel part of ATLAS, together with the electromagnetic barrel calorimeter, TileCal focuses on precise measurements of hadrons, jets, taus and the missing transverse energy $\left(E_{T}^{\text {miss }}\right)$. The performance requirements are driven by the ATLAS physics programme:

- The energy resolution for jets of $\sigma / E=50 \% / \sqrt{E(\mathrm{GeV})}$ $\oplus 3 \%$ guarantees good sensitivity for measurements of physics processes at the $\mathrm{TeV}$ scale, e.g. quark compositeness and heavy bosons decaying to jets. While one cannot separate the individual calorimeter performance issues, studies have shown that a random $10 \%$ non-uniformity on the TileCal cells energy response would add no more than $1 \%$ to the jet energy resolution constant term [4].

- For precision measurements such as the top quark mass, it will be desirable to reach a systematic uncertainty on the jet energy scale of $1 \%$. Since about a third of the jet transverse energy is deposited in TileCal [5], its energy scale uncertainty should ultimately be below a $3 \%$ requirement. 
- The response linearity within $2 \%$ up to about $4 \mathrm{TeV}$ is crucial for observing new physics phenomena (e.g. quark compositeness).

- A good measurement of $E_{T}^{\text {miss }}$ is important for many physics signatures, in particular for SUSY particle searches and new physics. In addition to sufficient total calorimeter thickness and a large coverage in pseudorapidity, this very sensitive measurement requires also a small fraction of dead detector regions which create fake $E_{T}^{\text {miss }}$. The requirement depends on the signal to background ratio of the search.

The Tile Calorimeter has been installed in the experimental hall since 2005 and since then has undergone through several phases of commissioning and integration in the ATLAS detector system. The main goal of this paper is to present the outcome of this commissioning phase, at the start of the LHC collisions data-taking. The paper is organised as follows: Section 2 gives a brief description of the Tile Calorimeter and discusses the overall detector status and the data-taking conditions after the commissioning was carried out. Section 3 presents the method for the channel signal reconstruction, the overall quality of the detector in coverage, noise characteristics and conditions stability. Section 4 shows the details on the three calibration systems used to set and maintain the cell energy scale and set the timing offsets, as well as results on the precision and stability of each system. The related energy scale uncertainties and the inter-calibration issues are also discussed. The last section (Sect. 5) is devoted to the validation of the performance using data from cosmic muons produced in cosmic ray showers in the atmosphere, referred to in short form throughout this paper as "cosmic muons" or "cosmic ray muons". Results are presented on energy and time reconstruction, uniformity across the calorimeter and comparison with Monte Carlo simulations. A subsection is devoted to the intercalibration of the scintillators that are located in the gap between barrel and extended barrels.

\section{Detector and data taking setup}

\subsection{Overview of the Tile Calorimeter}

TileCal is a large hadronic sampling calorimeter using plastic scintillator as the active material and low-carbon steel (iron) as the absorber. Spanning the pseudorapidity ${ }^{1}$ region $-1.7<\eta<1.7$, the calorimeter is sub-divided into the barrel, also called long barrel (LB), in the central region

\footnotetext{
${ }^{1}$ The pseudorapidity $\eta$ is defined as $\eta=-\ln \left(\tan \frac{\theta}{2}\right)$, where $\theta$ is the polar angle measured from the beam axis. The azimuthal angle $\phi$ is measured around the beam axis, with positive (negative) values corresponding to the top (bottom) part of the detector.
}

$(-1.0<\eta<1.0)$ and the two extended barrels (EB) that flank it on both sides $(0.8<|\eta|<1.7)$, as shown in Fig. 1 . Both the barrel and extended barrel cylinders are segmented into 64 wedges (modules) in $\phi$, corresponding to a $\Delta \phi$ granularity of $\sim 0.1$ radians. Radially, each module is further segmented into three layers which are approximately 1.5, 4.1 and $1.8 \lambda$ (nuclear interaction length for protons) thick for the barrel and 1.5, 2.6 and 3.3 for the extended barrel. The $\Delta \eta$ segmentation for each module is 0.1 in the first two radial layers and 0.2 in the third layer (Fig. 2). The $\phi, \eta$ and radial segmentation define the three dimensional TileCal cells. Each cell volume is made of dozens of iron plates and scintillating tiles. Wavelength shifting fibres coupled to the tiles on either $\phi$ edge of the cells, as shown in Fig. 3, collect the produced light and are read out via square light guides by two different photomultiplier tubes (PMTs), each linked to one readout channel. Light attenuation in the scintillating tiles themselves would cause a response non-uniformity of up to $40 \%$ in the case of a single readout, for particles entering at different impact positions across $\phi$. The double readout improves the response uniformity to within a few percent, in addition to providing redundancy.

In addition to the standard cells, the Intermediate Tile Calorimeter (ITC) covers the region $0.8<\eta<1.0$ (labelled D4 and C10 in Fig. 2). To accommodate services and readout electronics for other ATLAS detector systems, several of the ITC cells have a special construction: per side, three D4 cells have reduced thickness and eight $\mathrm{C} 10$ cells are plain scintillator plates. Located on the remaining, inner radius surface of the extended barrel modules, the gap scintillators cover the region of $1.0<\eta<1.2$ (labelled E1 and E2 in the figure), while the crack scintillators are located on the front of the Liquid Argon endcap and cover the region $1.2<\eta<1.6$ (labelled E3 and E4).

In the present (initial) configuration, eight pairs of crack scintillators have been removed to permit routing of signal cables from the 16 Minimum Bias Trigger Scintillators (MBTS), in each side. Located on the front face of the Liquid Argon end-cap cryostat, the MBTS span an $\eta$ range of $2.12<|\eta|<3.85$ and are readout by the TileCal EB electronics. They are used mainly for triggering on collisions in the very early stage of LHC operation and for rate measurements of halo muons, beam-gas and minimum bias events during the low-luminosity running.

The Tile Calorimeter readout architecture divides the detector in four partitions, a definition that is widely used in this paper. The barrel is divided in two partitions (LBA and LBC) by the plane perpendicular to the beam line and crossing the interaction point, and each of the two extended barrels is a separate partition (EBA and EBC).

The TileCal readout electronics is contained in "drawers" which slide into the structural girders at the outer radius of the calorimeter. Barrel modules are read out by two drawers 
Fig. 1 A cut-away drawing of the ATLAS inner detector and calorimeters. The Tile Calorimeter consists of one barrel and two extended barrel sections and surrounds the Liquid Argon barrel electromagnetic and endcap hadronic calorimeters. In the innermost radii of ATLAS, the inner detector (shown in grey) is used for precision tracking of charged particles
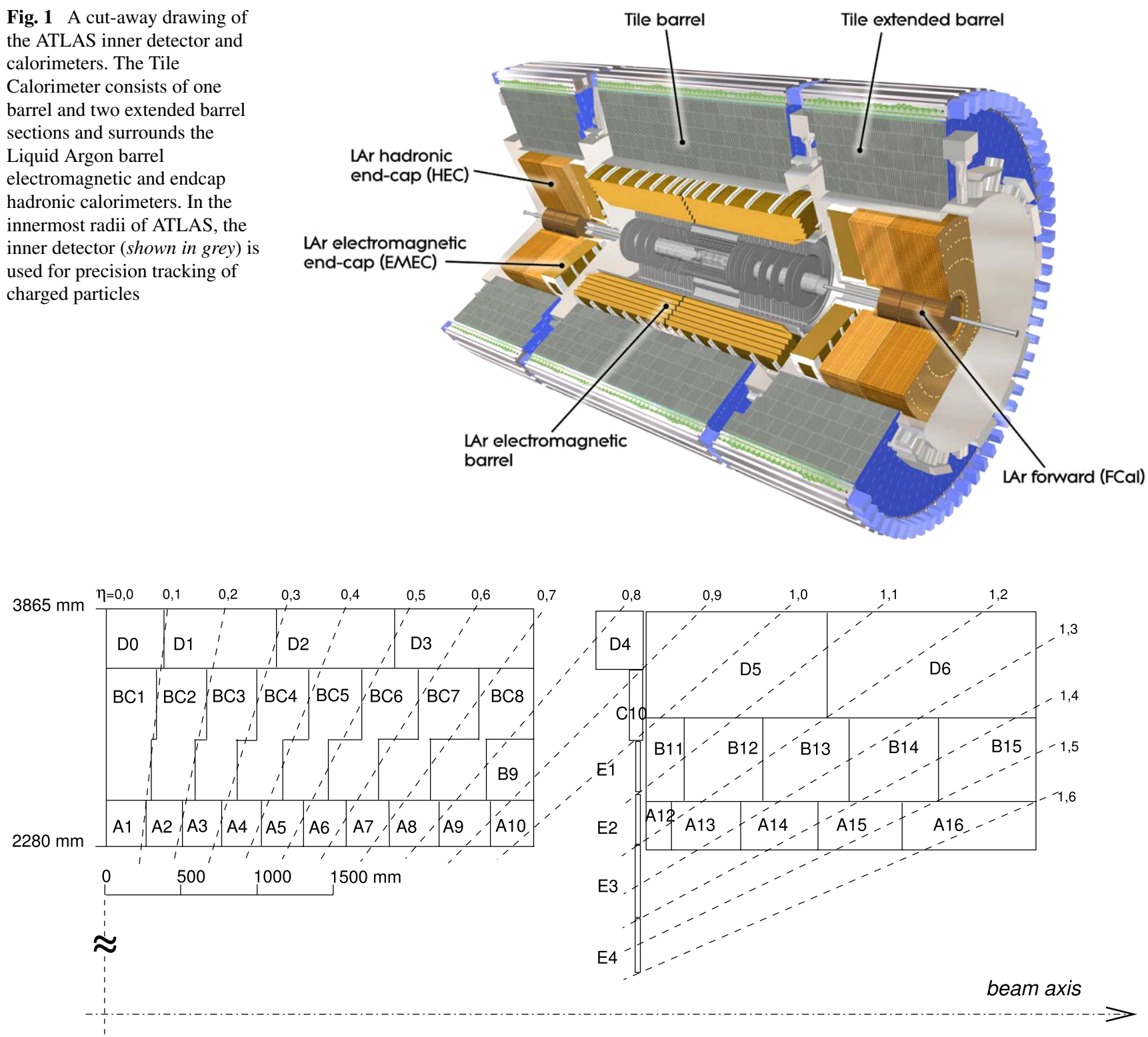

Fig. 2 Segmentation in depth and $\eta$ of the Tile Calorimeter modules in the barrel (left) and extended barrel (right). The bottom of the picture corresponds to the inner radius of the cylinder. The Tile Calorimeter is symmetric with respect to the interaction point. The cells between two consecutive dashed lines form the first level trigger calorimeter tower (one inserted from each face) and extended barrel modules are read out by one drawer each. Each drawer typically contains 45 (32) readout channels in the barrel (extended barrel) and a summary of the channels, cells and trigger outputs in TileCal is shown in Table $1 .^{2}$

The front-end electronics as well as the drawers' Low Voltage Power Supplies (LVPS) are located on the calorimeter itself and are designed to operate under the conditions

\footnotetext{
${ }^{2}$ The 16 reduced thickness extended barrel $\mathrm{C} 10$ cells are readout by only one PMT. Two extended barrel D4 cells are merged with the corresponding D5 cells and have a common readout.
}

of magnetic fields and radiation. One drawer with its LVPS reads out a region of $\Delta \eta \times \Delta \phi=0.8 \times 0.1$ in the barrel and $0.7 \times 0.1$ in the extended barrel.

In the electronics readout, the signals from the PMT are first shaped using a passive shaping circuit. The shaped pulse is amplified in separate high (HG) and low (LG) gain branches, with a nominal gain ratio of 64:1. The shaper, the charge injection calibration system (CIS), and the gain splitting are all located on a small printed circuit board known as the 3-in-1 card [6]. The HG and LG signals are sampled with the LHC bunch-crossing frequency of $40 \mathrm{MHz}$ using a 10-bit ADC in the Tile Data Management Unit (DMU) chip 


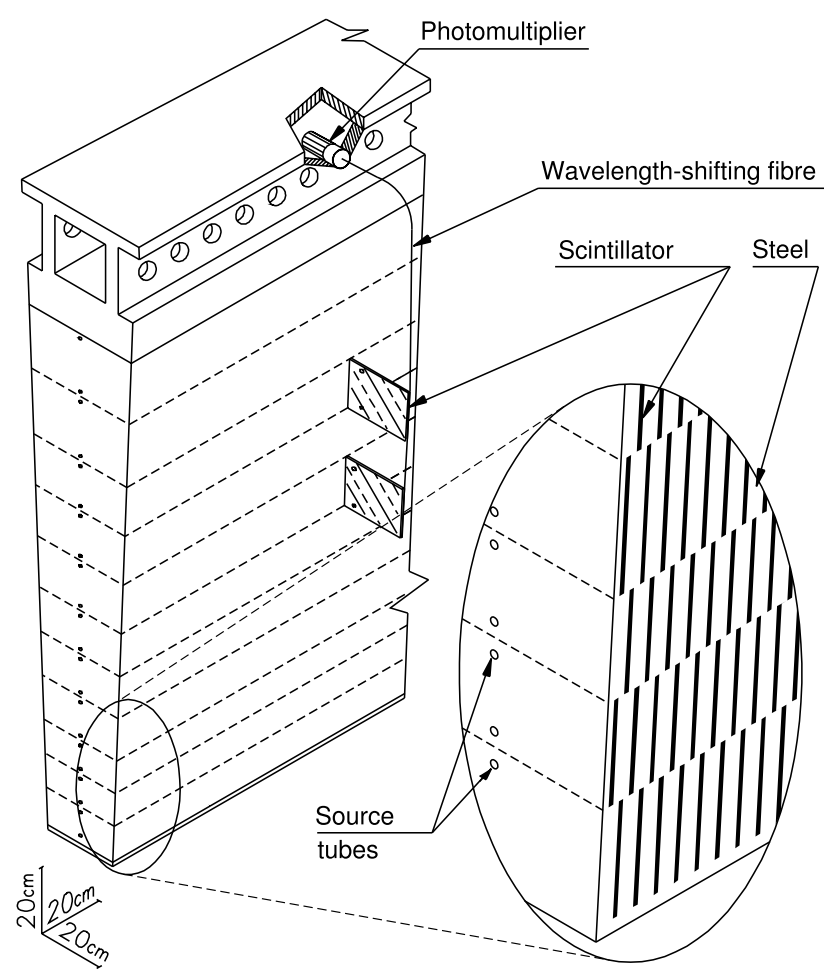

Fig. 3 Schematic showing the mechanical assembly and the optical readout of the Tile Calorimeter, corresponding to a $\phi$ wedge. The various components of the optical readout, namely the tiles, the fibres and the photomultipliers, are shown. The trapezoidal scintillating tiles are oriented radially and normal to the beam line and are read out by fibres coupled to their non-parallel sides

Table 1 Number of channels, cells and trigger outputs of the Tile Calorimeter. The gap and crack and MBTS channels are readout in the extended barrel drawers

\begin{tabular}{lcrc}
\hline & Channels & Cells & Trigger Outputs \\
\hline Long barrel & 5760 & 2880 & 1152 \\
Extended barrel & 3564 & 1790 & 768 \\
Gap and crack & 480 & 480 & 128 \\
MBTS & 32 & 32 & 32 \\
Total & 9836 & 5182 & 2080 \\
\hline
\end{tabular}

which is located on the digitiser board [7]. This chip contains a pipeline memory that stores the sampled data for up to $6.4 \mu$ s. The pipeline memory can be adjusted in coarse timing steps of $25 \mathrm{~ns}$. The digitisation timing of the ADCs can be adjusted in multiples of $\sim 0.1 \mathrm{~ns}$ so that the central sample is as close to the PMT pulse peak as possible and to make sure the full extension of the pulse is sampled. However, this adjustment is possible only for groups of six channels, so a residual offset remains, that must be dealt with at the signal reconstruction level (see Sect. 3.2). Due to bandwidth requirements, only seven samples from one gain are read out from the front-end electronics. A gain switch is used to determine if the high or low gain is sent. The digitised samples are sent via optical fibres to the backend electronics which are located outside the experimental hall. From the digitised samples, the back-end electronics determine the time and energy of the channel's signal as described in Sect. 3.2.

In addition to the digital readout of the PMT signal, a millisecond-timescale integrator circuit is also located on the 3-in-1 card. The Tile integrator is designed to measure the PMT current during ${ }^{137} \mathrm{Cs}$ calibrations (see Sect. 4) and also to measure the current from minimum bias protonproton interactions at the LHC. The integration period is approximately $14 \mathrm{~ms}$ and a 12-bit ADC is used for the readout.

Adder boards are distributed along the drawer. Each adder board receives the analogue signals from up to six 3in-1 cards corresponding to cells of the same $\eta$. The trigger signal corresponding to a "tower" (see Fig. 2) of cells with $\Delta \eta \times \Delta \phi=0.1 \times 0.1$ is formed by an analogue sum of the input signals and, together with the signals from the other calorimeters, are sent via long cables to the Level-1 (L1) calorimeter trigger system to identify jets, taus, total calorimeter energy and $E_{T}^{\text {miss }}$ signatures. The signal from all four gap and crack scintillators is also summed by the adder board and passed to the L1 calorimeter trigger. A second output of the adder boards (so-called muon output), that can be used at a later stage to reduce the muon background rates, contains only the signal from cells of the outermost calorimeter layer. Presently a fraction of the muon outputs is used for carrying the MBTS signals to the L1 trigger system.

\subsection{Detector and data taking overview}

The detector performance and stability results exposed in this paper are based on calibration systems' data and random triggered events which cover extended periods from mid-2008 up to the end of 2009 excluding the maintenance period between December 2008 and May 2009. The results from cosmic muons and single beam are from the autumn 2008 data-taking period, with the exception of the single beam data for timing studies, for which the winter 2009 and spring 2010 data is also used.

The Tile Calorimeter at the end of 2008 data-taking period was fully operational with approximately $1.5 \%$ dead cells. The majority of the dead cells were due to three drawers that were non-operational because of power supply problems or data corruption, amounting to 60 cells or $1.2 \%$. The remaining dead cells were randomly distributed throughout TileCal. During the 2009 data-taking period there were 48 unusable cells, fewer than $1 \%$. The number of dead $\mathrm{L} 1$ trigger towers is less than $0.5 \%$ and they are uniformly distributed throughout the detector. For details on how nonoperational cells are defined and the breakdown of their problems for the 2009 data-taking, see Sect. 3.1. 
The cosmic data used for performance validation was collected mainly between September and October 2008 using the full ATLAS detector, including the inner detector and muon systems, with around one million events used for the present paper. The cosmic trigger configuration during this run period consisted of L1 triggers from the muon spectrometer $^{3}$ (both the Resistive Plate Chamber (RPC) and the Thin Gap Chambers (TGC)), the L1 calorimeter trigger and the MBTS. For much of the cosmic ray analysis discussed in Sect. 5, the data sample was selected by requiring a L1 trigger and at least one track reconstructed in the inner detector, from the Pixel, SemiConductor Tracker (SCT) and Transition Radiation Tracker (TRT) ${ }^{4}$ The majority of the events came from the L1 muon spectrometer triggers. During this running period, the ATLAS magnets were run in four different configurations; no magnetic field, solenoid magnet on only, toroid magnet on only and both solenoid and toroid magnets on. The results exposed here were obtained with the full ATLAS fields on.

From the single beam data used in this paper the "splash" events and "scraping" events are used for time and energy studies. The former term is used for events occurring when the LHC beam hits the closed tertiary collimators positioned $140 \mathrm{~m}$ up-stream of the detector and are characterised by millions of high-energy particles arriving simultaneously in the ATLAS detector. The latter occur when the open collimators are scraping the LHC beam, allowing a moderate number of particles to the detector.

\section{Detector performance and signal reconstruction}

\subsection{Detector and data quality status overview}

The TileCal detector operated at the end of 2009 with $99.1 \%$ of cells functional for the digital readout and $99.7 \%$ of trigger towers functional for the L1. The numbers and fractions of non-operational cells, channels and trigger towers in the four calorimeter partitions are shown in Table 2.

The problematic channels belong to two categories: channels with fatal problems and channels with data quality problems. The so-called fatal problems are channels deemed unusable and are masked for the offline reconstruction and at the High Level Trigger (HLT). These channels include:

1. 44 cells (88 channels) due to two drawers with nonfunctional LVPS.

2. 10 channels with no response due to failures of one or more components in the readout chain, such as 3-in-1 cards, PMTs or ADCs.

\footnotetext{
${ }^{3}$ See Ref. [2], Fig. 1.4, for details on the layout.

${ }^{4}$ See Ref. [2], Fig. 1.1, for details on the layout.
}

Table 2 Summary of the number of masked channels and cells in TileCal as of November 9th, 2009. The number of dead trigger towers quoted is towers that are non-operational due to problems in TileCal's front-end electronics, not counting those related to LVPS (18 towers)

\begin{tabular}{lrrl}
\hline Partition & $\begin{array}{l}\text { Masked } \\
\text { Channels }\end{array}$ & \multicolumn{1}{l}{$\begin{array}{l}\text { Masked } \\
\text { Cells }\end{array}$} & $\begin{array}{l}\text { Dead Trigger } \\
\text { Towers }\end{array}$ \\
\hline Barrel A-side & $59(2.05 \%)$ & $23(1.60 \%)$ & $2(0.3 \%)$ \\
Barrel C-side & $58(2.01 \%)$ & $25(1.74 \%)$ & $0(0.0 \%)$ \\
Ext. barrel A-side & $6(0.29 \%)$ & $0(0.00 \%)$ & $2(0.5 \%)$ \\
Ext. barrel C-side & $1(0.05 \%)$ & $0(0.00 \%)$ & $1(0.3 \%)$ \\
Total & $124(1.26 \%)$ & $48(0.93 \%)$ & $5(0.3 \%)$ \\
\hline
\end{tabular}

3. 24 channels with digital data errors (17 channels with a high occurrence rate of corrupted data and 7 with gain switching problems).

4. 2 channels with high noise

The position in $(\eta, \phi)$ as of November 2009 of the unusable masked cells as described above, are shown in Fig. 4 and are summarised in Table 2. One can notice the majority of the masked cells concentrated in two non-functional front-end drawers.

Channels with data quality problems are flagged as such for the reconstruction, but they are not masked. These channels include:

1. Channels with occasional data-corruption problems, mainly due to front-end electronics malfunction or bad configuration. These are excluded from the reconstruction by checking a quality fragment in the data record on

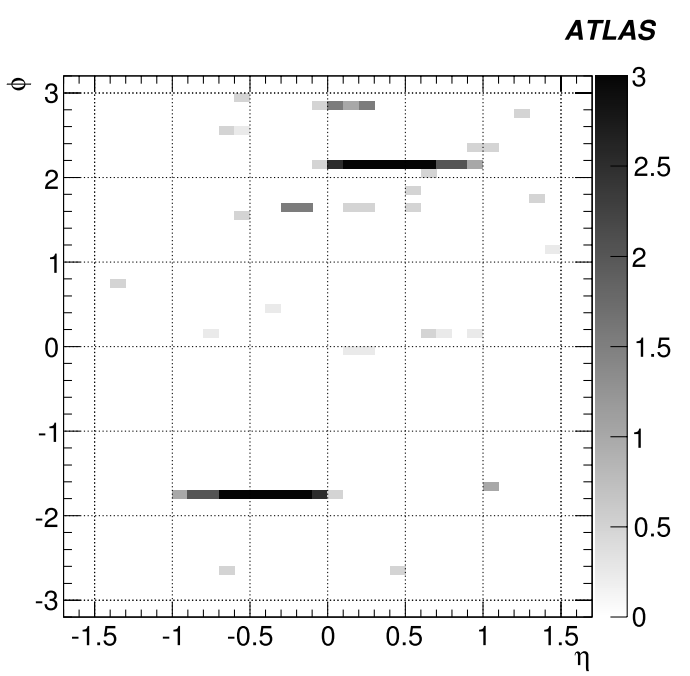

Fig. 4 Position in $\eta$ and $\phi$ of the masked cells representing the status on November 9th, 2009. The colours corresponding to numbers $1,2,3$ show the number of layers masked for this $(\eta, \phi)$ region. The non-integer numbers indicate that one readout channel of the cell is masked 
an event by event basis. A fraction of the channels can be recovered by resetting the front-end between LHC fills.

2. Channels which cannot be calibrated with one of the calibration systems (see Sect. 4). These are flagged as poorly calibrated channels.

3. Noisy channels, which are treated by describing appropriately in the database their higher-than-average noise level to take into account while reconstructing their energy.

4. Channels where the response varies significantly over time. These are also flagged for the offline use as poor quality channels but their response can be corrected over time if the source of variation is understood. Typical cases include channels with varying response due to changes over time of the high voltage applied to the photomultipliers.

The parameters that directly affect the measured response of a channel are the temperature in the drawer and the applied high voltage because the PMT gain depends on them. The PMT gain $G$ is proportional to $V^{7}$, where $V$ is the applied high voltage (HV), and decreases with temperature by $0.2 \%$ per ${ }^{\circ} \mathrm{C}$. The operating conditions of the detector have been constantly monitored online and recorded by the Detector Control System (DCS). The operating values of voltages, currents, temperatures at the LVPS and at the frontend have been very stable. Figure 5 gives a measure of the long term evolution of the high voltage applied on the PMTs for two periods of 3 and 6 months separated by the maintenance period. The HV values, which are typically close to $\sim 670 \mathrm{~V}$, have shown on average a difference of $0.17 \mathrm{~V}$ with respect to the value set during intercalibration with an RMS of $0.37 \mathrm{~V}$ during the considered period. This average stability within $0.4 \mathrm{~V}$ for the whole calorimeter represents a $0.4 \%$ reproducibility in the gain of the PMTs due to this factor alone. Figure 6 shows the stability of the temperature measured by a probe installed in one PMT block for the same period as for the $\mathrm{HV}$ measurements. The average over all the calorimeter PMT probes is $24.1^{\circ} \mathrm{C}$ with an $\mathrm{RMS}$ of $0.2^{\circ} \mathrm{C}$ for a period of 9 months interleaved by the maintenance period.

\subsection{Energy and time reconstruction}

The channel signal properties_-pulse amplitude, time and pedestal—for all TileCal channels are reconstructed with
Fig. 5 Stability of the PMT high voltage with respect to its set value, averaging over all PMTs for two periods of 3 and 6 months (left) separated by the maintenance period. The distribution of the differences of the measured and the set HV values for all PMTs over the period considered is also shown (right)
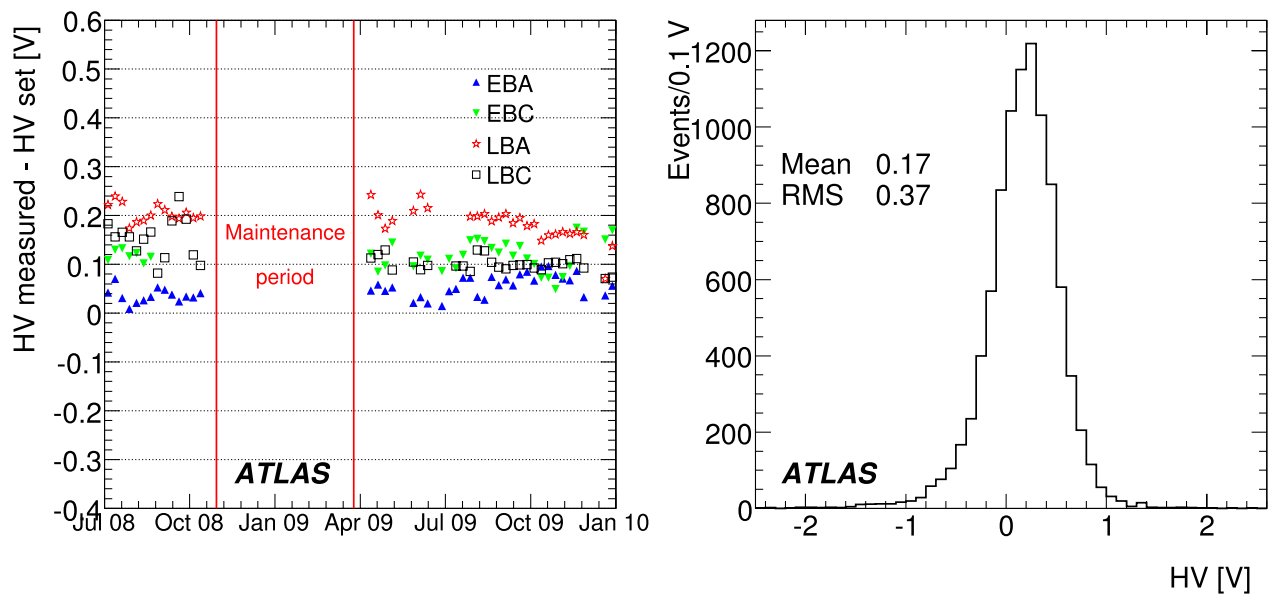

Fig. 6 Stability of the temperature, as measured at one PMT in each drawer, averaging over all drawers and presented for two periods of 3 and 6 months separated by the maintenance period (left). The distribution of the values for individual drawers over the whole period is also shown (right)

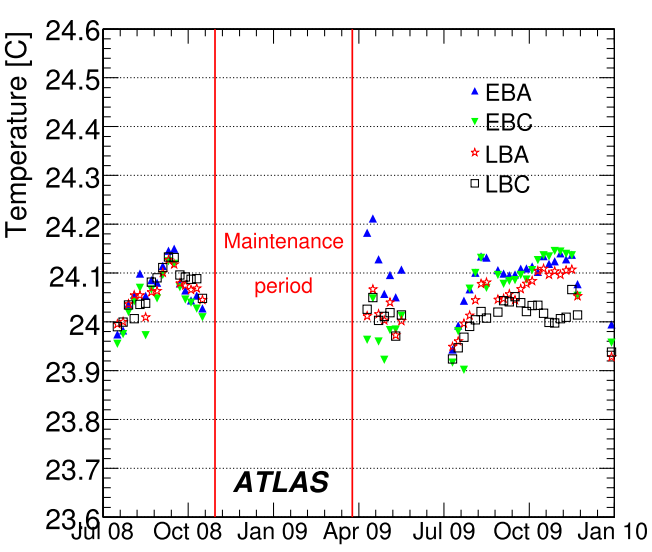


the Optimal Filtering (OF) method [8], which makes use of weighted linear combinations of the digitised signal samples (spaced by $25 \mathrm{~ns}$ ). Due to the simplicity of its mathematical formulation, OF is implemented in the Digital Signal Processors (DSPs) of the ReadOut Driver boards (RODs) [9] and therefore provides energy and time information to the HLT of ATLAS during the online data-taking. At present, since the data-taking rate allows it, the seven digitised samples are also available offline for all the events together with the results of the OF reconstruction from the RODs. The procedure to compute the energy (given by the amplitude $A)$ and time $(\tau)$ are given by the equations:

$A=\sum_{i=1}^{n=7} a_{i} S_{i}, \quad \tau=\frac{1}{A} \sum_{i=1}^{n=7} b_{i} S_{i}$,

where $S_{i}$ is the sample taken at time $t_{i}(i=1, \ldots, n)$. The coefficients of these combinations, $a_{i}$ and $b_{i}$, known as the OF weights, are obtained from knowledge of the pulse shape and noise autocorrelation matrix, and are chosen in such a way that the impact of the noise to the calorimeter resolution is minimised. Figure 7 shows the pulse shape extracted from data taken at the testbeam, selecting a channel with a given value of deposited energy for each gain. This pulse shape is the reference used in the estimation of the OF weights.

The reconstructed channel energy used by the HLT and offline is:

$$
E_{\text {channel }}=A \cdot C_{\mathrm{ADC} \rightarrow \mathrm{pC}} \cdot C_{\mathrm{pC} \rightarrow \mathrm{GeV}} \cdot C_{\mathrm{Cs}} \cdot C_{\mathrm{Laser}} .
$$

The signal amplitude $A$, described in more detail above, represents the measured energy in ADC counts as in (1). The factor $C_{\mathrm{ADC} \rightarrow \mathrm{pC}}$ is the conversion factor of ADC counts to charge and it is determined for each channel using a well defined injected charge with the CIS (Charge Injection System) calibration system. The factor $C_{\mathrm{pC} \rightarrow \mathrm{GeV}}$ is the conver-

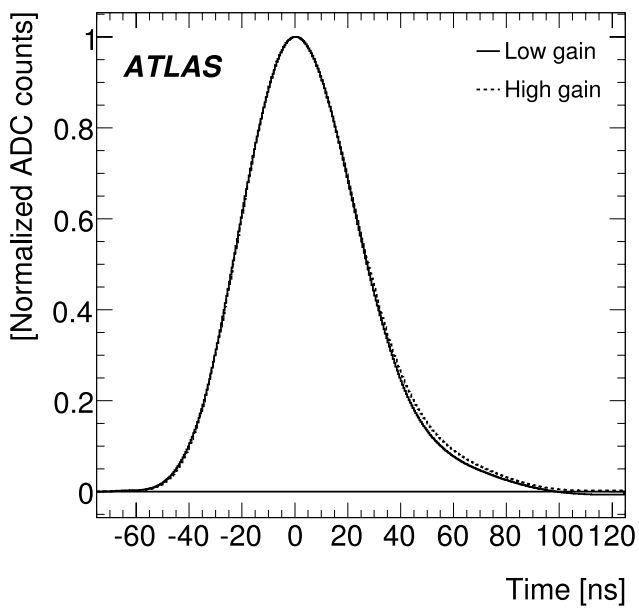

Fig. 7 Pulse shape for high and low gain from testbeam data, used as reference for the OF weights calculation sion factor of charge to energy in $\mathrm{GeV}$ and it has been defined at testbeam for a subset of modules via the response to electron beams of known momentum in the first radial layer. This factor is globally applied to all cells after being adjusted for a dependence on the radial layer (see Sect. 4.4). The factor $C_{\mathrm{Cs}}$ corrects for residual non-uniformities after the gain equalisation of all channels has been performed by the Cs radioactive source system. The factor $C_{\text {Laser }}$, not currently implemented, corrects for non-linearities of the PMT response measured by the Laser calibration system. The derived time dependence of the last two factors will be applied to preserve the energy scale of TileCal. The details of the calibration procedures are discussed in Sect. 4.

The channel time, $\tau$ in (1), is the time difference between the peak of the reconstructed pulse and the peak of the reference pulse. The OF weights used in the reconstruction were calculated based on this reference pulse shifted by a time phase that depends on each channel's timing offsets measured with the calibration systems (and single-beam data), the time-of-flight from the interaction point to that cell and the hardware time adjustments mentioned in Sect. 2.1. Thus the reconstructed time $\tau$ should be compatible with zero for energy depositions coming from the interaction point. If the time residual is not well known, for small deviations $(|\tau|<15 \mathrm{~ns})$ the uncertainty of the reconstructed amplitude depends on $\tau$ through a well-defined parabolic function, that can be used for an energy correction at the level of the HLT or offline reconstruction.

The OF results rely on having, for each channel, a fixed and known time phase between the pulse peak and the $40 \mathrm{MHz}$ LHC clock signal. This is not the case during the commissioning phase of the detector, where signals caused by cosmic rays are completely asynchronous with respect to the LHC clock. Nevertheless OF can still be applied in this case and an accurate reconstruction may be obtained by applying the proper weights for each event according to the time position of the signal. The estimation of the signal time is achieved through an iterative procedure provided by a set of $\mathrm{OF}$ weights calculated at different phases from $-75 \mathrm{~ns}$ to $+75 \mathrm{~ns}$ in steps of $1 \mathrm{~ns}$. Figure 8 presents the relative difference between the reconstructed offline energy and the energy calculated in the DSPs for cosmic muon data and shows the effect of the limited numerical precision of the DSPs. The results in the following sections are based on channel energies reconstructed offline with the iterative procedure to define the phase.

The Fit method is another signal reconstruction algorithm. It is based on a three parameter fit to the known pulse shape function $g(t)$, as expressed by:

$S_{i}=A g\left(t_{i}-\tau\right)+p e d$.

The meaning of the variables $S_{i}$ and $t_{i}$ and the parameters $A$ and $\tau$ is the same as for the OF method, while ped is a 


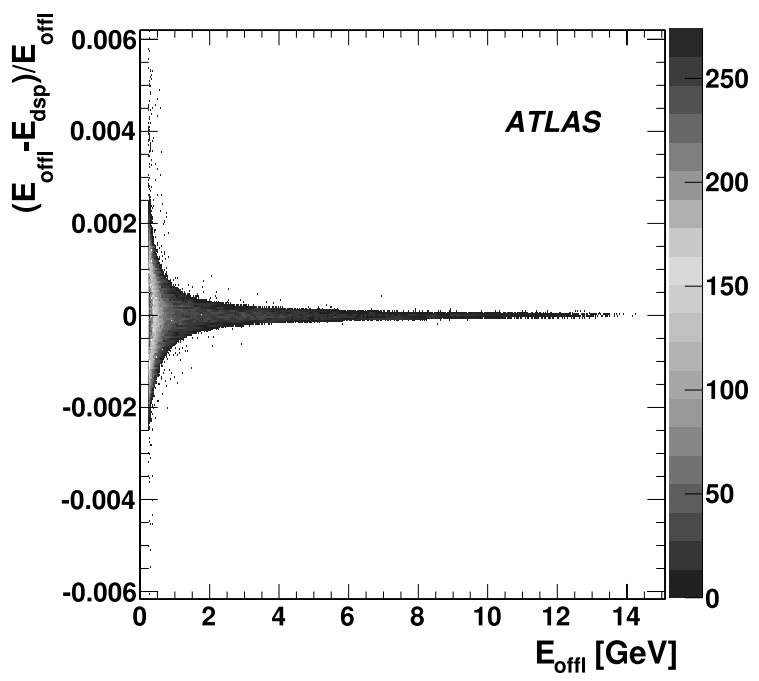

Fig. 8 Difference between the reconstructed offline energy, $E_{\text {offl }}$, and the energy given by the DSP $E_{\mathrm{DSP}}$ relative to $E_{\mathrm{offl}}$ and as a function of $E_{\text {offl }}$ (in $\mathrm{GeV}$ ), extracted from cosmic muon runs

free parameter that defines the baseline of the pulse. The Fit method is mathematically equivalent to OF in the absence of pile-up and noise, but it is not suitable for fast online signal processing in DSPs. Results from the Fit and OF methods were compared with testbeam data and were found to be equivalent [10]. Since the autumn of 2008 data-taking, the Fit method is used only for CIS calibration data, where the pulse is a superposition of charge-proportional and chargeindependent components [10].

The cell energy is the sum, and the cell time the average, of the respective measurements by the two corresponding readout channels. In cases of single readout cells, or if one of the channels is masked out, the cell energy is twice the energy measured in the single available channel. The measurement of the cell's energy is thus robust to failures in a single readout channel.

\subsection{Noise performance}

The noise in TileCal was measured in dedicated bi-gain standalone runs with empty events (often called pedestal runs) and in random triggered events within ATLAS physics runs (often called random triggers). The noise of each channel was derived from the seven digitised samples using the same method that was used for signal reconstruction in cosmic and single beam events, i.e. using the OF with iterations. ${ }^{5}$ In Fig. 9 the evolution during the running periods of 2008 and 2009 of the average noise, in ADC counts, is

\footnotetext{
${ }^{5}$ Note that the level of noise depends on the OF method used. The noniterative OF method results in lower noise than the OF with iterations by $\sim 14 \%$. Note also that the non-iterative OF will be applied for the data-taking during the collision phase, since the timing will be fixed by the LHC $40 \mathrm{MHz}$ clock frequency.
}

shown for all channels and for an individual channel. The channel noise is estimated as the RMS of the single digitised samples averaged over the events in dedicated TileCal pedestal runs. The overall stability is better than $1 \%$.

The cell noise in $\mathrm{MeV}$ as a function of $\eta$ is shown in Fig. 10 averaged over all modules in $\phi$ for cells in a given $\eta$ position. The cell noise is estimated as the RMS of the cell's energy distribution using the iterative OF signal reconstruction in random triggered events during a physics run with LHC single beam in 2008. Different colours are used to indicate cells in different longitudinal layers. The noise values vary between 30 and $60 \mathrm{MeV}$. The channels with higher noise are principally at the proximity of the LVPS which are located at the outer boundaries of the TileCal barrel and extended barrel modules.

The cell noise probability distribution is an important component in the ATLAS calorimeter's energy clustering algorithm. It is determined from the cell energy in empty events recorded through the standard ATLAS data acquisition chain within physics runs and it is characterised by the $\sigma$ of a fitted single Gaussian to the energy $(E)$ distribution. The ratio $E / \sigma$ is used to judge if a cell has a noise-like or a signal-like energy deposition. Figure 11 shows the ratio $E / \sigma$ for all TileCal cells (squares). One can observe the existence of non-Gaussian tails that could lead to fake signal cells if a criterion of $E / \sigma>4$ is used. However, since a double Gaussian distribution provides a good description of the data, the two Gaussian $\sigma$ 's and the relative amplitudes are used to construct a probability density function on the basis of which a new "effective $\sigma$ " $\left(\sigma_{\text {eff }}\right)$ for every cell is defined at the significance level of $68.3 \%$. The improvement is shown in Fig. 11 where the triangles represent the ratio $E / \sigma_{\text {eff }}$ for

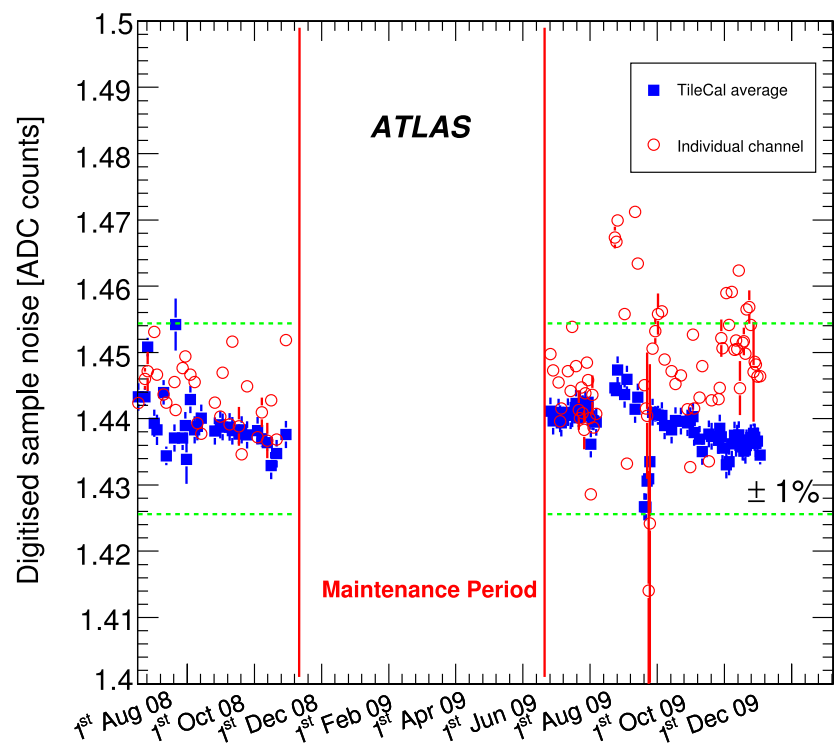

Fig. 9 Stability of average noise (RMS of the single digitised samples averaged over events and channels), in ADC counts, for all channels and for an individual channel 


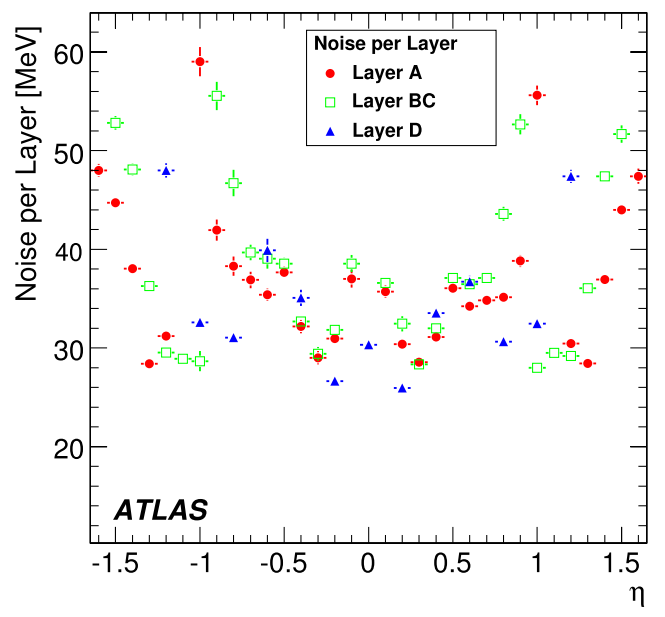

Fig. 10 Average cell noise in random triggered events as a function of the cell $\eta$ and radial layer. The noise is represented by the RMS of the cell's energy distribution and the error bar shows its spread over all cells in the same pseudorapidity bin

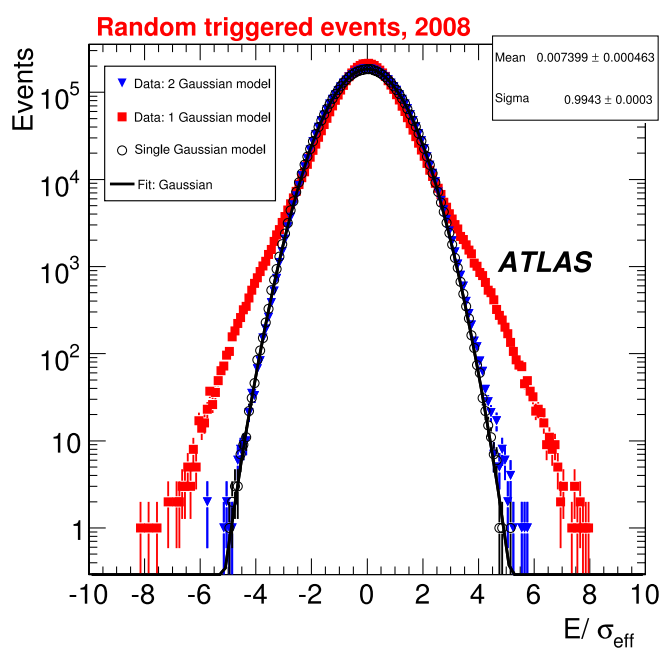

Fig. 11 Significance level of the cell energy as compared to noise (Energy/Gaussian $\sigma$ ) using the single and the double Gaussian descriptions of noise in random triggered events

all the calorimeter cells. One can observe that there are no tails when compared to a Gaussian fit (line) or to a toy Monte Carlo noise generator, that randomly attributes to cells energies from a single Gaussian model (circles). Thus the ratio $E / \sigma_{\text {eff }}$ can be safely used to distinguish signal from noise in a TileCal cell.

\section{Calibration}

This section describes the calibration procedures and data sets used in TileCal to establish the reference detector response. Furthermore, the calibration results obtained in the years 2008 and 2009, during the commissioning of the Tile
Calorimeter in the ATLAS cavern, and the cross-checks related to the current understanding of its calibration are also discussed. The main objectives of the calibration procedures in TileCal are to:

- Establish the global electro-magnetic (EM) scale and the uncertainty associated with it. The EM scale calibration factor converts the calorimeter signals, measured as electric charge in $\mathrm{pC}$, to the energy deposited by electrons, which would produce these signals.

- Minimise, measure and correct the cell-to-cell variations at the EM scale.

- Measure and correct the non-linearity of the calorimeter response.

- Measure the average time offset between the signal detection and the collision time for every readout channel.

- Monitor the stability of these quantities in time.

The Tile Calorimeter calibrations systems treat different sections of the readout chain as illustrated in Fig. 12. They provide:

- Calibration of the initial part of the signal readout path (including the optics elements and the PMTs) with movable radioactive ${ }^{137} \mathrm{Cs} \gamma$-sources [11], hereafter to be called simply Cs.

- Monitoring of the gains of the photomultipliers by illuminating all of them with a laser system $[4,12]$.

- Calibration of the front-end electronic gains with a charge injection system (CIS) [6].

In order to detect non-uniformities or degradation in the detector elements (optical and otherwise), the calibration systems are specified to meet a precision of $1 \%$ on the measurement of the response of a cell.

The number of channels that cannot be calibrated by each individual calibration system is well below $1 \%$. This is additional to the number of channels that are unusable due to LVPS problems or other issues not related to the given calibration system. In the following sections the performance distributions appear sometimes with fewer channels due to the fact that not all could be available for all the calibration periods.

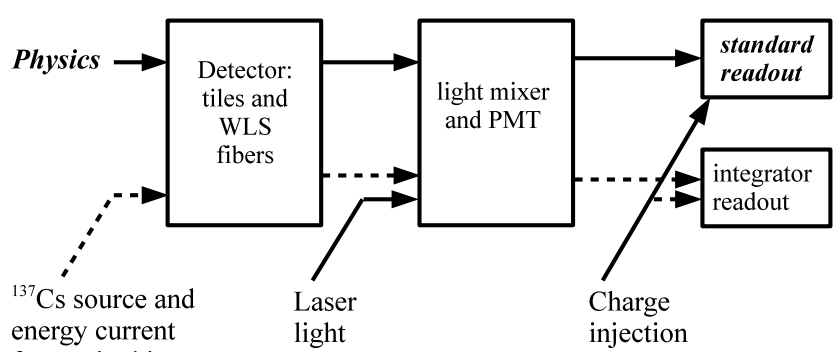

from min. bias

Fig. 12 Flow diagram of the readout signal paths of the different TileCal calibration tools. The paths are partially overlapping, allowing for cross-checks and an easier identification of component failures 
The current calibration protocol includes a number of dedicated calibration runs performed with a frequency derived from experience gained during the detector commissioning. The CIS constants are very stable in time and are only updated twice per year. For monitoring and identification of bad channels, CIS runs are performed between physics runs twice per week. For monitoring, laser runs are also performed twice per week. The resulting laser constants will be used only for monitoring purposes until the stability of this calibration system is fully understood. The Cs scans are performed outside beam periods, with a periodicity of weeks or months, depending on the machine schedule since a full scan takes 6 to 8 hours. Starting from 2010, every Cs run is expected to result in new constants that adjust the global EM energy scale which will be updated accordingly. Laser runs accompany Cs runs in order to disentangle between changes related to the optical system and PMTs. Since the laser runs are more frequent than the Cs scans, the former provide information on the PMT gain changes between two Cs scans.

A dedicated monitoring system based on slow integrators [6] records signals in the Tile readout channels over thousands of bunch crossings during the physics runs and is also a part of the Tile calibration framework. As this measurement requires experience with collisions it is still being commissioned.

\subsection{Charge injection system and gain calibration in the readout electronics}

The circuitry for the Charge Injection system is a permanent part of each front-end electronics channel [6] and it is used to measure the $\mathrm{pC} / \mathrm{ADC}$ conversion factor for the digital readout of the laser calibration and physics data and to determine the conversion factor for the slow integrator readout, measured in ohms.

To reconstruct the amplitude for each injected charge, a three-parameter fit is performed as described at the end of
Sect. 3, with the amplitude being one of the parameters of the fit [10]. To determine the values of the gains for each channel, dedicated CIS calibration runs are taken frequently, in which a scan is performed over the full range of charges for both gains. The typical channel-to-channel variation of these constants is measured to be approximately $1.5 \%$, as shown in Fig. 13. This spread indicates the level of corrections for which the CIS constants are applied.

The stability in time of the average high gain and low gain readout calibration constants from August 2008 to October 2009 is shown in Fig. 14 for $99.4 \%$ of the total number of ADCs. The time stability of a typical channel is also shown for each gain. Over this period, the RMS variation for the high and low gain detector-wide averages and for the single channels shown, is less than $0.1 \%$. The superimposed bands of $\pm 0.7 \%$ represent the systematic uncertainty for the individual channel calibration constants, mainly due to the uncertainty on the injected charge.

The distributions of high gain and low gain readout calibration constants for individual ADC channels were compared for the sample of channels calibrated during the TileCal standalone testbeam period of 2002 to 2003 and for the full detector in the cavern in 2009. No significant change in the calibration constants was observed, thus limiting the contribution from the CIS calibration to the systematic uncertainty on transferring the EM scale from testbeam to ATLAS to below $0.1 \%$.

To determine the values of the gains for each channel for the current integrator readout, dedicated calibration runs are periodically taken, in which a scan is performed over the full range of currents for all six integrator gains. The channel gain is extracted as a slope from a 2-parameter fit performed on the measured channel response in voltage to each applied current. The typical channel-to-channel variation of these integrator gain constants is measured to be approximately 0.9\%, as shown in Fig. 15 (left) for the gain used during calorimeter calibration with the Cs radioactive source. The 12-bit ADCs used to digitise the PMT currents were pro-
Fig. 13 Channel-to-channel variation of the high gain (left) and of the low gain (right) readout calibration constants as measured by the CIS, prior to any correction. The measured HG/LG gain ratio of 62.9 corresponds to the nominal of 64 (see Sect. 2.1) within tolerances of individual electronics components

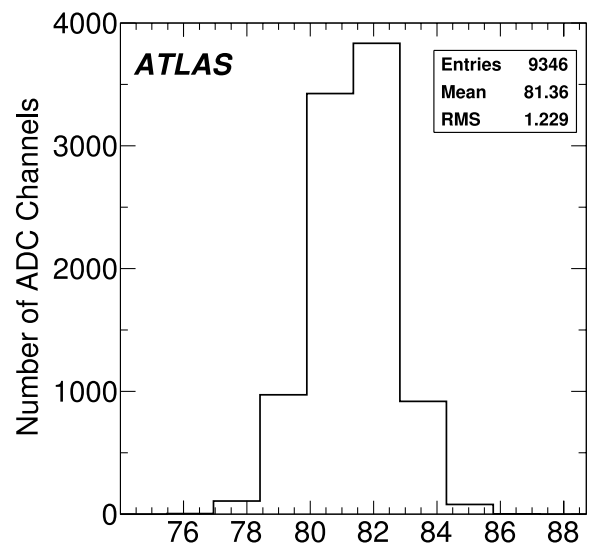

High- gain Calibration [ADC counts / pC]

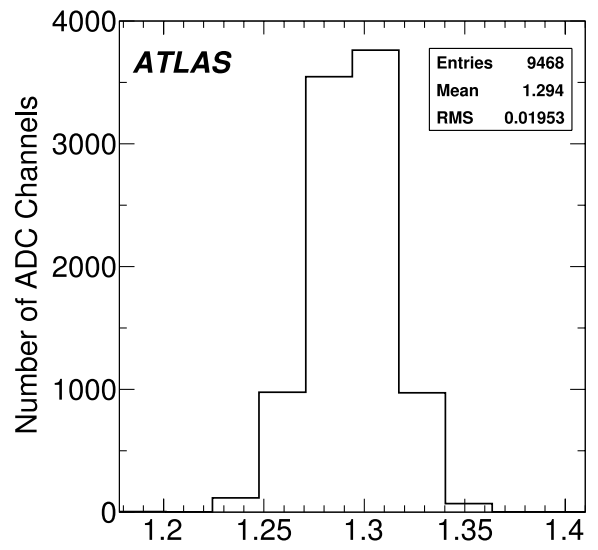

Low- gain Calibration [ADC counts / pC] 
Fig. 14 Stability in time of the average high gain (left) and low gain (right) readout calibration constants from August 2008 to October 2009

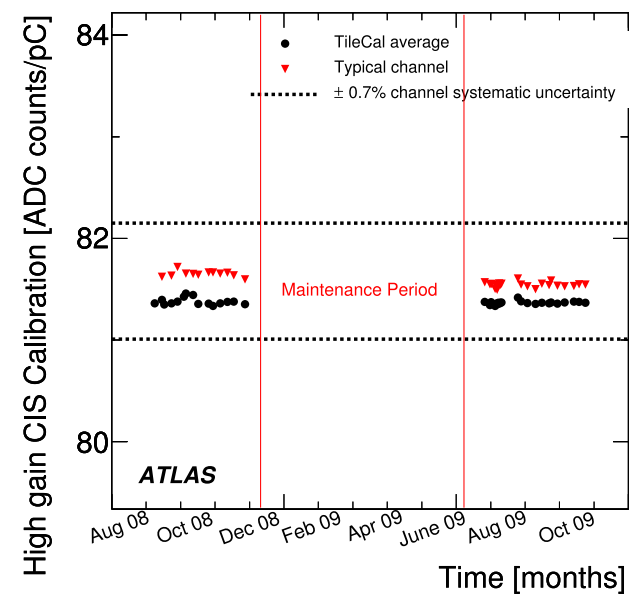

duced in two unequal batches with about $2 \%$ difference in amplifier gains, which can be clearly seen in the distribution of the integrator gains in Fig. 15 (left).

The relative variation of the integrator gains used by the Cs calibration system is shown in the right part of Fig. 15. The measurements in $95.9 \%$ of the integrators performed at different dates are compared to the reference measurements of January 2008. The error bars represent the dispersion of the individual channel measurements relative to their reference values in the first run. The stability of individual channels is better than $0.05 \%$ while the stability of the average integrator gain is better than $0.01 \%$ over the considered period of time of 26 months.

The variation of the integrator gains for individual channels used in the Cs calibration system readout from 2001 to 2009 was studied on the sample of channels calibrated in both instances. No significant change in the calibration constants was observed over eight years. The contribution from the integrator gain calibration to the systematic uncertainty on setting the EM scale of TileCal in ATLAS as compared to the testbeam was found to be below $0.2 \%$.

\subsection{Calibration with laser system}

The Tile Calorimeter is equipped with a custom-made laser calibration system [12] dedicated to the monitoring and calibration of the Tile photomultiplier properties, including the gain and linearity of each PMT. The frequency doubled infrared laser providing a $532 \mathrm{~nm}$ green light beam is located in the ATLAS USA15 electronics room, $100 \mathrm{~m}$ from the detector. The laser emits short pulses, which reasonably resemble those from the physics signals, with a nominal energy of a few $\mathrm{mJ}$. This power is sufficient to simultaneously saturate all Tile readout channels, and thus to probe their linearity over the full readout dynamic range. A dedicated set of optical elements insures proper attenuation, partial de-coherence and propagation of the original light beam to every photomultiplier used in the Tile Calorimeter readout. This calibration system was commissioned until September 2009 and since then it is operating in a stable configuration. By varying the voltages applied to the photomultipliers it was shown that the system sensitivity to the relative gain variations is of $0.3 \%$ on data sets recorded over few hours. The long term stability of the laser calibration system is under study.
Fig. 15 Distribution of the integrator gain used by the Cesium calibration system is shown on the left. Relative stability over twenty-two months of the same integrator gain is shown on the right
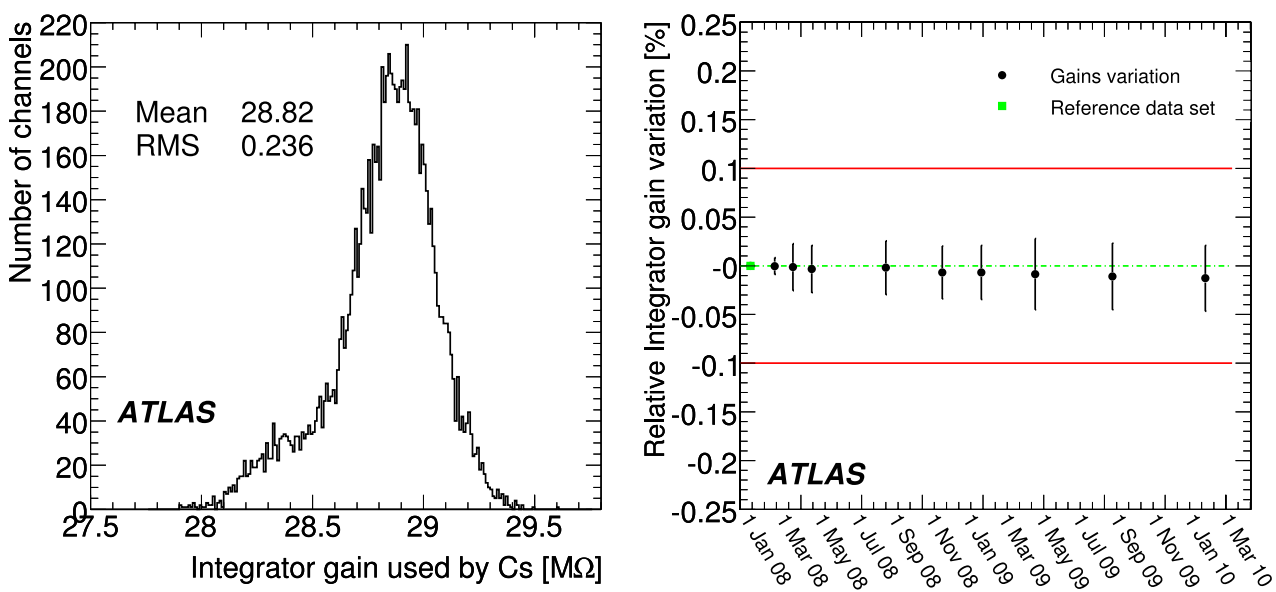
The time stability of the PMT gains was evaluated using dedicated laser runs and averaging over $98.8 \%$ of the Tile$\mathrm{Cal}$ channels. An estimation of the relative gain variation in time was based on the analysis of the shape of the distribution of the PMT responses to the signal induced by the laser system at many instances. The average gain variation as a function of time over 40 days is shown on Fig. 16. This variation is found to be within $1.0 \%$ over the considered period of time. The displayed error bars of $0.5 \%$ account for both the statistical uncertainty and the systematic effects and are entirely dominated by the latter. The systematic uncertainty comes from the limited reproducibility of the light intensity on the photomultipliers downstream of the full optical chain through which the laser beam propagates to the detector. The design goal of the laser system is to monitor the relative gain stability with $0.5 \%$ accuracy for time periods of months to years. The results mentioned above set the precision with which the PMT response stability can be monitored by the laser system between two Cs scans that are typically one month apart and monitor the combined response of the optics elements and PMTs.

Once the global variation of the laser signal is accounted for, the gain stability per individual channel can be studied. A typical channel to channel variation for $\mathrm{HG}$ and $\mathrm{LG}$ is shown in Fig. 17, where the relative gain variations for two laser calibration runs separated by 50 days are presented. The shaded sidebands represent channels with relative variation above $1 \%$. The observed RMS of $0.3 \%(0.2 \%)$ in the $\mathrm{HG}(\mathrm{LG})$ is a convolution of residual fluctuations of the laser system and variations of the PMT response. Therefore, this RMS can be considered as an upper limit on possible stochastic variations in photomultiplier gains.

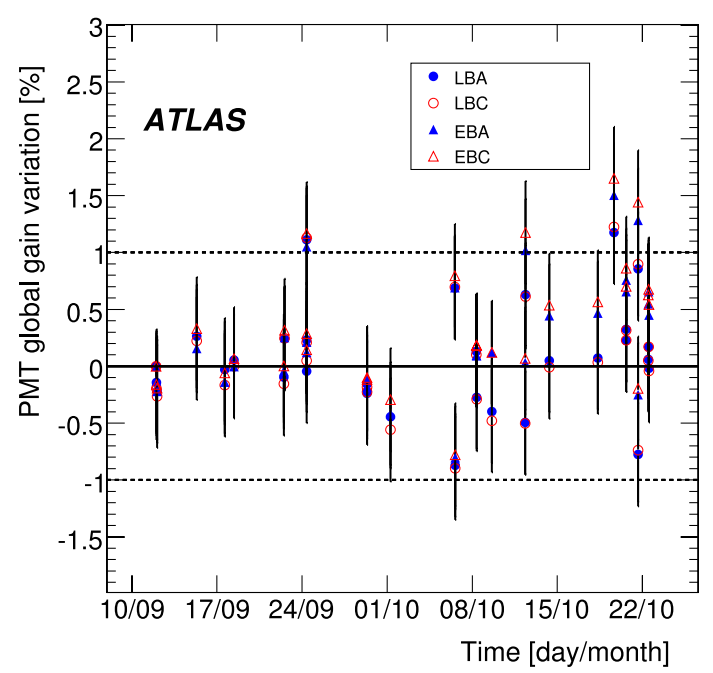

Fig. 16 Average PMT gain variation measured by the laser calibration system as a function of time over forty days in 2009
Once the intrinsic stability of the laser calibration system is understood, this system will be used to calibrate the gain and linearity ${ }^{6}$ of each PMT.

\subsection{Calibration based on ${ }^{137} \mathrm{Cs}$ radioactive $\gamma$-source}

The Tile Calorimeter includes the capability of moving through each scintillator tile a Cs radioactive $\gamma$-source along the $Z$-direction of the ATLAS detector. Capsules containing the Cs sources with activities of about $330 \mathrm{MBq}$ emitting $0.662 \mathrm{MeV} \gamma$-rays are hydraulically driven through a system of $10 \mathrm{~km}$ of steel tubes that traverses every scintillating tile in every module [13]. Three sources of similar intensity are deployed in the three cylinders of the Tile Calorimeter. When a capsule traverses a given cell, the integrator circuit located on the 3-in-1 cards (Sect. 2.1), reads out the current signal in the PMTs. The total area under the integrator current vs capsule position curve corresponding to the source path length in a cell, is calculated and normalised to the cell size. This estimator of the cell response to Cs is used throughout this section.

Source scans provide the means to diagnose optical instrumentation defects [14] and to measure the response of each individual cell. The precision of the Cs based calibration was evaluated from the reproducibility of multiple measurements under the same conditions and was found to be about $0.3 \%$ for a typical cell [11]. The precision is $0.5 \%$ for the cells on the edge of the TileCal cylinders and a few percent for the narrow cells $\mathrm{C} 10$ and D4 in the gap region (see Fig. 3). As discussed in Sect. 5.4, cosmic ray muons are used to cross-check the calibration factors for the cells of this type.

\subsubsection{Intercalibration and EM scale factor via the Cs system}

The Cs calibration has proceeded in two distinct phases.

- Photomultiplier gain equalisation to a chosen level of Cs response was performed for every individual channel on the $11 \%$ of production TileCal modules that were tested with particle beams during 2001-2004 [10]. The next step was to measure the numerical value for the fixed EM scale with electron beams. With the electrons entering the calorimeter modules at an incidence angle of $20^{\circ}$, the average cell response normalised to beam energy was measured to be $(1.050 \pm 0.003) \mathrm{pC} / \mathrm{GeV}$, defining the TileCal EM scale factor. This factor was determined for the cells of the first layer and propagated via the gain equalisation

\footnotetext{
${ }^{6}$ All the photomultipliers used in TileCal were characterised on their arrival from Hamamatsu at dedicated test benches with LED light sources. No PMT was found with non-linearity worse than $3 \%$ up to $800 \mathrm{pC}$ of collected charge.
} 
Fig. 17 Channel-to-channel variation of the relative gain of the photomultipliers for two Laser calibration runs taken in HG (LG) mode, shown on the left (right)

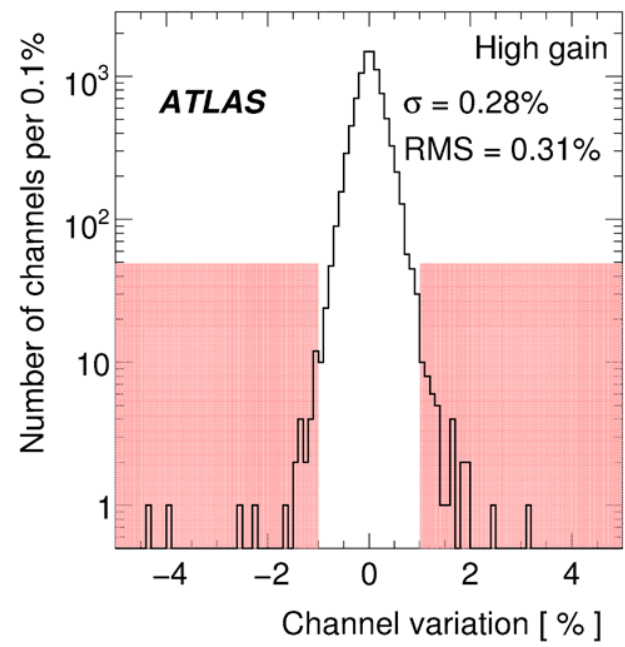

to all the other cells. The RMS spread of $(2.4 \pm 0.1) \%$ was found to be due to local variations in individual tile responses and tile-fibre optical couplings. The above two steps effectively resulted in setting the cell EM scale in the subset of TileCal modules exposed to electron beams.

- The second phase in the calibration was to reproduce the above PMT gain equalisation on the full set of the Tile Calorimeter modules in the ATLAS environment and to transfer via the Cs response the EM scale factor as defined in the testbeam. This took place in the second half of 2008. In some cases the PMT gain is intentionally higher by $20 \%$ (D0, D1, D2, D3, D4 and C10 cells) in order to improve on signal to noise ratio for the detection of muons (see also Sect. 5.1). For the central barrel cells of the third radial layer this improvement will facilitate their possible usage in the L1 muon trigger. The EM scale for these cells is recovered by applying appropriate corrections to the cell energy reconstruction.

To set the EM scale as defined at the testbeam, the target response to Cs for 2008 and 2009 was defined as the response measured at the testbeam scaled by the ratio of the activities of the testbeam source to the sources used in the cavern. These ratios were measured by intercalibrating the sources using two TileCal modules that are kept outside the experimental hall. The source activity decay time between the testbeam and the ATLAS scans was taken into account. By adjusting PMT gains in order to have equal response to Cs between the testbeam and the ATLAS setup, the numerical factor that converts charge to $\mathrm{GeV}$ is preserved. It is evident that the comparison of the source activities is of utmost importance in order to preserve the absolute energy scale as set with electrons.

Five ${ }^{137} \mathrm{Cs}$ radioactive sources of different ages and activities were used over the last years. Three sources are currently used in the ATLAS cavern and two different sources were used for checks on instrumentation quality and for the calibration at the testbeam. In spring of 2009, one long barrel and one extended barrel module were scanned sixty times under the same conditions with all five radioactive sources. With the reproducibility of a single measurement better than $0.1 \%$, a full set of ratios of the source activities was evaluated with the precision of $0.05 \%$. The results for these ratios after averaging over all data sets available are shown in the last column of Table 3. It should be noted that the third column of the table gives an initial estimation of the activities as measured by the manufacturer with a $\pm 15 \%$ uncertainty. We plan to exchange the sources between the Tile Calorimeter cylinders in the cavern for future checks on reproducibility of the responses and also to monitor the ratios of the source activity in time.

\subsubsection{Effect of magnetic field}

Comparing the EM scale response between the testbeam and full detector, the magnetic field configuration has to be considered. During the testbeam no magnetic field was present while during data-taking in ATLAS, TileCal operates in the presence of magnetic field. The calorimeter iron, mainly the

Table 3 Activity of five ${ }^{137}$ Cs radioactive sources as of April 2009, and ratios with respect to the reference source RP3713 of the measured activities averaged over all data sets collected in the spring of 2009. Source RP3713 was used in calibrations during the test beam period. Source RP3712, kept in Building 175, is used for ageing tests

\begin{tabular}{llll}
\hline Source & $\begin{array}{l}\text { Location in } \\
2009\end{array}$ & $\begin{array}{l}\text { Activity in } \\
\text { April } 2009( \pm 15 \%)\end{array}$ & $\begin{array}{l}\text { Measured activity, } \\
\text { normalised to RP3713 }\end{array}$ \\
\hline RP3713 & Storage & $264 \mathrm{MBq}$ & - \\
RP4091 & LB & $372 \mathrm{MBq}$ & $1.1860 \pm 0.0005$ \\
RP4090 & EBA & $363 \mathrm{MBq}$ & $1.1590 \pm 0.0005$ \\
RP4089 & EBC & $377 \mathrm{MBq}$ & $1.2180 \pm 0.0007$ \\
RP3712 & Bld. 175 & $319 \mathrm{MBq}$ & $1.2200 \pm 0.0005$ \\
\hline
\end{tabular}


Fig. 18 Ratio of the TileCal cell response to the radioactive Cs source in full ATLAS magnetic field to the TileCal cell response to the Cs source without the field, shown as function of $\eta$ (left). Ratio of the TileCal D3 cell response to radioactive Cs source in full ATLAS magnetic field over its response to the Cs source without the field, shown as function of $\phi$ (right). The vertical lines indicate the position of the ATLAS toroid coils

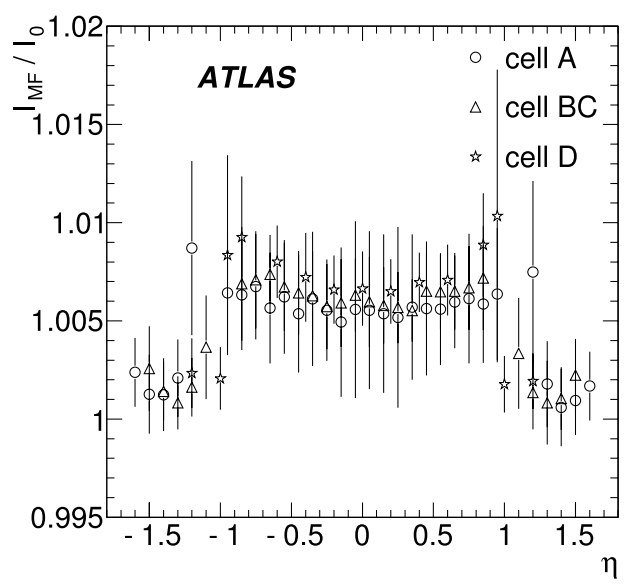

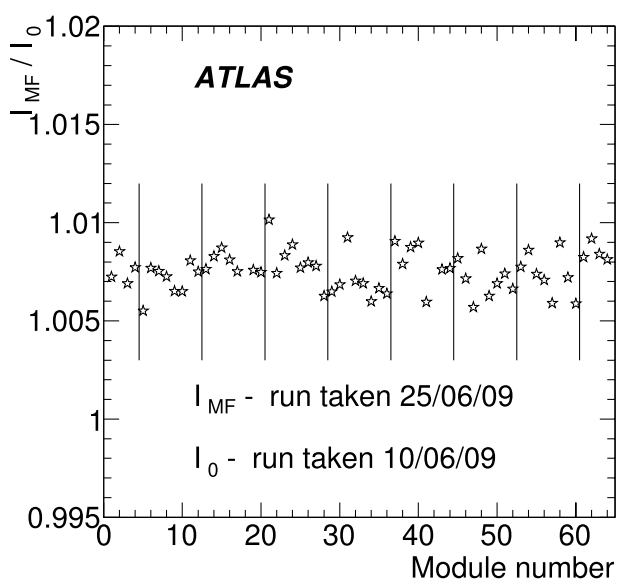

girder volume at the outer radius, serves as the flux return of the solenoid field. The general behaviour of iron-scintillator calorimeters in magnetic field is known from other experiments [15-17]. A small increase in the scintillator light yield, which also varies modestly over a broad range of the applied field is expected.

The impact of the full ATLAS magnetic field on the Tile Calorimeter response was studied using the Cs calibration system. The ratio of the TileCal cell response to a radioactive Cs source in the full ATLAS magnetic field to its response to the Cs source without the field is given in Fig. 18 (left) as a function of $\eta$ for two consecutive Cs runs. The cells in individual radial layers are shown with different symbols. The error bars represent the RMS of the above ratio over the sample of the sixty four identical cells in the full $\phi$ range.

As expected, the effect of magnetic field is stronger in the barrel partitions, where the flux of the solenoid field return is the most intense, and where the increase in calorimeter response is on average $\sim 0.6 \%$. A small increase of $\sim 0.2 \%$ is observed for the extended barrel. This increase was not fully reproducible in every instance of the magnetic field turn-on in 2008, which contributes $0.5 \%$ to the systematic uncertainty of propagating the EM scale from the testbeam to the ATLAS running configuration. The ratio of the $\mathrm{D} 3$ cell $^{7}$ response to radioactive Cs source with and without the full ATLAS magnetic field is shown in the right part of Fig. 18 as function of $\phi$. The vertical lines illustrate the positions of the Toroid coils. No clear structure in $\phi$ is observed, indicating that in the final ATLAS configuration the full magnetic field does not significantly affect the Tile Calorimeter response uniformity in $\phi$. Starting from 2010, Cs calibrations will be exclusively based on the data taken with the full magnetic field.

\footnotetext{
${ }^{7} \mathrm{~A}$ cell through which the Toroid field return is the strongest.
}

\subsubsection{Monitoring with Cs in ATLAS}

Once the EM scale was established and reproduced in ATLAS, periodic scans are performed to monitor the stability of the detector response to the radioactive source in time. This is the final step that insures the monitoring of the known EM scale in time.

The Tile Calorimeter response to the Cs source as a function of time is shown in Fig. 19. The first scan was taken approximately two weeks after the original PMT gain equalisation in July 2008. Around 55 calibration runs with the radioactive source are considered for the time period from August 2008 to February 2010. The maintenance period of

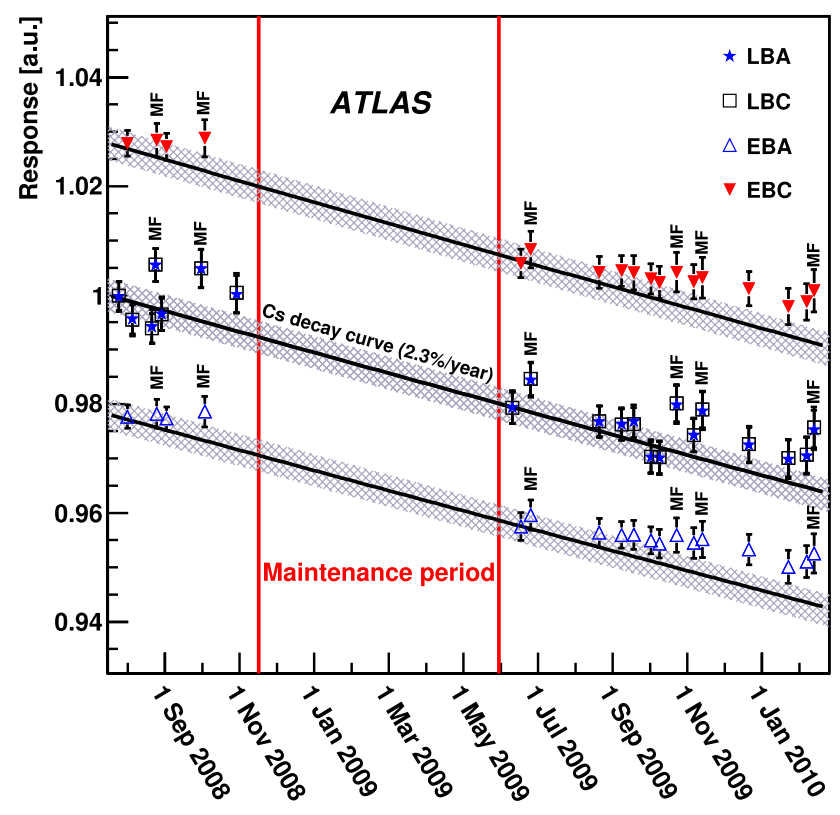

Fig. 19 TileCal response to radioactive $C s$ sources in all four calorimeter partitions not corrected for the difference in the source activities as a function of time, averaging over all channels in a partition. The error bars represent the RMS spread in the responses of the sample of channels used. The "MF" symbol stands for the Cs calibration data taken with magnetic field on 
six months is indicated by the vertical lines on Fig. 19 and is excluded from the studies. The very first points after the maintenance period correspond to the second gain equalisation to the same target value, corrected for the expected decrease in the source activity in time, as indicated on the figure. The average response to the radioactive sources in the four calorimeter partitions is shown by the points of different colours. Since three sources with about $3 \%$ difference in their activity are used in the barrel and two extended barrel cylinders, the data points follow three distinct paths in time. The error bars, which are always below $0.4 \%$, represent the RMS spread in responses over the full set of channels in a given partition. The number of cells with unreliable Cs calibration or with unstable HV level is below $0.2 \%$ of the total and they are excluded from the present study. The shaded bands along the lines indicate the level of reproducibility of the Cs measurements. The "MF" label indicates that the corresponding Cs calibration run was taken with both the ATLAS toroid and solenoid fields on. The response increase due to magnetic field is larger in the barrel partitions. Details on the magnetic field effects were already discussed in Sect. 4.3.2.

The relative deviation of the measured Cs response from the expected values due to the decrease in the source activity is shown in Fig. 20 (left) for the same set of the calibration runs reported above. Similarly, the maintenance period is excluded and the "MF" marks are used when the magnetic field was present during the calibration. The overall TileCal

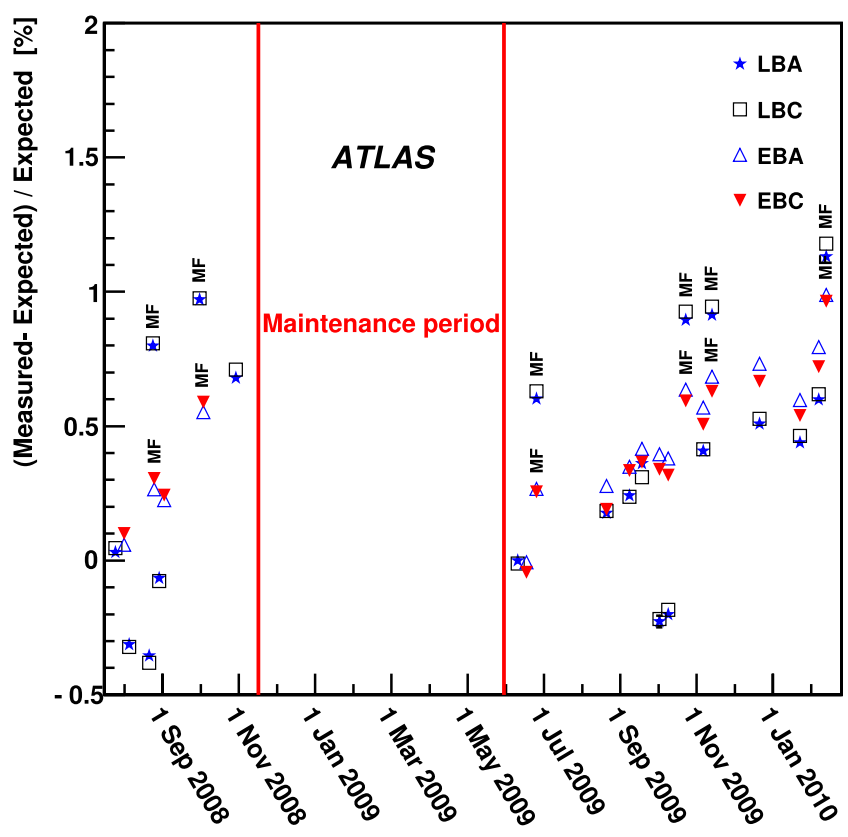

Fig. 20 Relative deviations of the TileCal response to Cs sources from the expected value for all four calorimeter partitions, shown as a function of time (left). The ratio of RMS/mean of the TileCal response to radioactive Cs source in all four calorimeter partitions is shown as response to the radioactive sources follows the expected Cs decay within $1 \%$ when no magnetic field is applied. Within this $1 \%$, there is a visible deviation from the expected decay line with increasing average response over time. A study of the Cs calibration procedure has been unable to attribute this increase to any subtle systematic effect, therefore it is attributed to an increase in the detector response and it is under investigation. A conservative time dependent systematic uncertainty on the calibration of the EM scale of about $0.1 \%$ per month is adopted to account for this effect. It is estimated from the Cs data with no magnetic field within two periods of 3 and 7 months in 2008, 2009 and 2010. The ratio of RMS/mean of the TileCal response to radioactive Cs sources in all four calorimeter partitions is shown as function of time in Fig. 20 (right). The spread in the measured Cs responses stays within $0.4 \%$ over seventeen months indicating that the cell-to-cell intercalibration does not significantly change over this period of time. A small effect of the magnetic field on the Cs response spread is also clearly seen.

\subsection{Calorimeter intercalibration}

In this section the understanding of the cell and layer intercalibration acquired from the testbeam and from calibration and single beam data is exposed. The intercalibration as validated by cosmic muons is exposed later in Sect. 5.3.1.

The intercalibration with Cs sources in the ATLAS cavern reports channel response non-uniformities at the level of

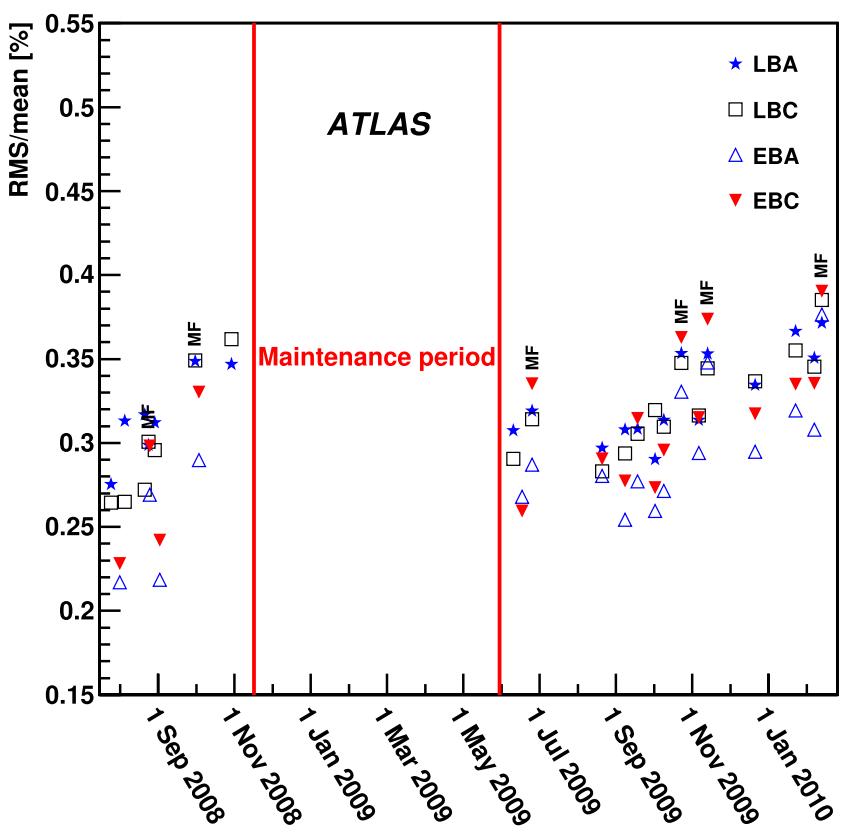

function of time (right). The "MF" symbol stands for the Cs calibration data taken in the magnetic field. The response is averaged over the channels of each partition 
Fig. 21 The average energy measured in the single beam events recorded in September 2008. Left: average energy measured in individual radial layers after the radial layer corrections were applied ( $\mathrm{A}$ is the inner radius layer). Right: the average energy measured in individual partitions, demonstrating good intercalibration between them.

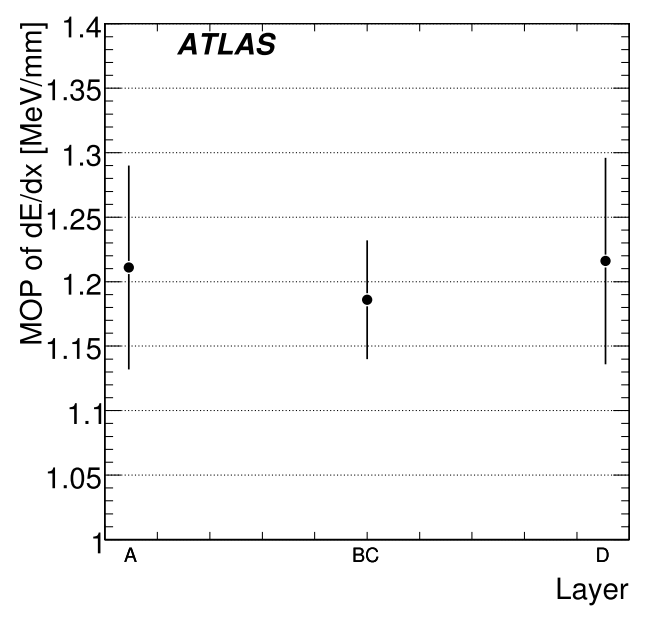

$\sim 0.4 \%$ in each Cs-scan, which is compatible with the precision of this calibration system. Since the Cs system uses a different readout path than what is used for the physics signal induced by particles (digital readout), other calibration uncertainties also have to be considered. The charge injection system reports negligible non-uniformity after the channel-to-channel corrections. The integrators contribute at the level of $0.05 \%$, which is negligible. Altogether, the non-uniformities are given mostly by the Cs system. The Cs scans of the whole Tile Calorimeter revealed the same level of uniformity among individual optical elements in a cell as was measured during the optics instrumentation period.

In the testbeam, a difference in the response to the $\mathrm{Cs}$ source and to particles was observed, increasing for layers at larger radius [10]. This is due to the increasing size of the scintillator tiles for the external layers, and the resulting few percent layer miscalibration is accounted for by applying radial depth weights in the energy scale calibration. The details of this procedure are described in Ref. [18]. Figure 21 (left) shows the $\mathrm{d} E / \mathrm{d} x$ for muons crossing the calorimeter parallel to the beam axis along its whole length from scraping events ${ }^{8}$ in 2008 . The $\mathrm{d} E / \mathrm{d} x$ response for the muons from single beam events was estimated as the peak of the fit to the convolution of a Landau function with a Gaussian (most probable value, referred throughout the paper as MOP). Within a large statistical uncertainty, the response vs radial layer is flat. Given the fact that if the radial depth weights had not been applied the ratio of responses between layers A and D would be 1.088, this observation gives confidence in their use. Figure 21 (right) shows the mean response of the four TileCal partitions to muons. Data are from 2008 single beam runs. The precision is limited by the systematic uncertainty of $\sim 4 \%$, while the statistical uncertainty is $\sim 2 \%$.

${ }^{8}$ Events produced by the proton beam hitting the edge of the collimators located at about $140 \mathrm{~m}$ upstream ATLAS.
4.5 Uncertainty on the propagation of the EM scale from testbeam

The EM scale of TileCal in ATLAS is set by adjusting the PMT HV to reproduce the calorimeter response to the Cs radioactive source to the level it had during the tests with electron beams, where the EM scale was determined and measured. ${ }^{9}$ After correcting for the expected decrease in the Cs source intensity, the HV levels currently set in ATLAS are expected to reproduce those used at the testbeam. Any difference in the detector parameters from that observed at the testbeam, if not fully understood or disproved and if it affects the EM scale setting, should be considered as the systematic uncertainty on the EM scale determination.

The following sources of systematic uncertainties on the EM scale, as discussed in the previous sections, are only related to the transfer of the EM calibration factor from the testbeam to ATLAS because they originate from differences between the two setups:

- $0.1 \%$ from the calibration of the digital readout $(\mathrm{HG}, \mathrm{LG})$ by CIS.

$-0.2 \%$ from the calibration of the Cs readout gains.

$-0.5 \%$ from the non-reproducibility of the calorimeter response after the magnetic field is turned off, as reported by Cs measurements in 2008 (see Sect. 4.3.2).

$-0.3 \%$ from the uncertainty to the radial depth weights, briefly mentioned in Sect. 4.4.

The first two uncertainties were evaluated by comparing calibration results on a fixed sample of channels which were calibrated during the testbeam and then re-calibrated recently in ATLAS. The two latter are related to observations with limited understanding of the underlying phenomena.

\footnotetext{
${ }^{9}$ The modules that were calibrated with the beams were carefully chosen to give a representative sample of the full TileCal module population. Thus no significant uncertainty on the EM scale is expected to result from data obtained with the electron beams.
} 
When these uncertainties are combined in quadrature with the statistical uncertainty on the EM scale derived at the testbeams, the result is a systematic uncertainty of $\pm 0.7 \%$.

In addition to the above, there is a systematic uncertainty from the observed increase of the calorimeter response to the Cs source with respect to the expected value by about $0.1 \%$ per month as observed during 10 months of frequent monitoring in 2008 and 2009-2010. This is a time dependent uncertainty increasing since the initial EM scale setting in ATLAS in June 2008.

- Presently (early 2010) we assign an uncertainty of $-1.5 \%$ due to the increasing response of roughly $0.1 \% /$ month as measured by the Cs system during 2008 to 2010 .

- During the data-taking period from which cosmic muon results are presented in this paper (September to October 2008), the same uncertainty was $-0.8 \%$.

After setting the EM scale in ATLAS, the high voltage values applied on the PMTs were compared between the testbeam periods (2002 to 2004) and June 2008. While the TileCal response has been calibrated reliably with the Cs system to match the response measured during beam calibrations and hence to transfer EM scale to the ATLAS cavern, the PMT high voltages for the LB partition in June 2008 had to be lowered on average by $(6.5 \pm 0.2) \mathrm{V}$ compared to those used during testbeam calibrations. This was due to the fact that the Cs system measured an increased response in June 2008 for the beam calibrated modules with respect to their response in testbeams. If this response increase had not been a detector effect but an artifact of the Cs calibration system, a corresponding bias of $-5.3 \%$ (the true energy being higher than the measured one) would have to be considered as an uncertainty for the cosmic data taken in autumn 2008. This would be added to the uncertainty from the observed increase of roughly $0.1 \%$ per month since June 2008, as mentioned above.

The energy response from muons is a handle to assess this uncertainty or bias. A full description on the energy scale analysis with cosmic and testbeam is given in Sect. 5.3. The comparison between the testbeam and ATLAS EM scale is performed via the double ratio of $\mathrm{d} E / \mathrm{d} x$ Data/MC ratios of cosmic over testbeam muons for LB modules. In other words, the agreement of data to the MC energy scale between testbeam and ATLAS is compared. Table 6 presents the values and the uncertainties of the above mentioned double ratio per layer. Among the calibration related uncertainties, the contributions from the non-reproducibility of the response increase due to magnetic field and from the unexplained response increase measured by the Cs during 2008 are comprised. The reported ratios show an agreement of the EM scale set in 2008 and the expected scale as it was transported from the testbeam within the uncertainty range.
However, the possible calibration bias mentioned in the previous paragraph, that would be represented by a double ratio of 0.95 , can be excluded only at a $\lesssim 2 \sigma$ level.

If the uncertainty coming from the reduced high voltage settings with respect to the testbeam is not taken into account, the overall estimate of the EM scale systematic uncertainty from the calibrations is $(-1.7 \%,+0.7 \%)$ in early $2010 .{ }^{10}$

\subsection{Timing calibration}

To allow for optimal reconstruction of the energy deposited in the calorimeter by the OF signal reconstruction method (see Sect. 3), the time difference between the digitising sampling clock and the peak of the PMT pulses must be minimised and measured with a precision of $1 \mathrm{~ns}$. To achieve this, the clock phases in the DMUs in the front-end hardware (see Sect. 2.1) are adjusted in multiples of $0.1 \mathrm{~ns}$. Ideally all PMT signals would be sampled at the peak but several factors limit the ability to do this. First, the clock phase is defined per digitiser board which corresponds to six readout channels. Second, only one clock phase can be defined for both gains and there is a $2.3 \mathrm{~ns}$ difference between the HG and LG pulse peaks. Therefore in the front-end hardware, the accuracy of phase synchronisation for individual channels is limited to be within 3 ns. Any residual time differences between the clock phase and the pulse peak are measured for each channel and accounted for in the OF signal reconstruction algorithm.

The time phase and the residual offsets for all channels can be measured using the laser calibration system, cosmicray events, beam splash and collision events. What is exposed in this section is the procedure to only pre-set the timing in order to synchronise the detector with the trigger signals and with the other detectors prior to the final detailed adjustments, to be carried out with collisions data.

Prior to beam, the laser was the primary source used to measure the channel timing. Since the laser light is asynchronous with respect to the clock, a single reference channel in each partition was selected and all other channels' timing was defined with respect to that reference [19]. The timing precision for channels in the same module is $0.6 \mathrm{~ns}$ for $99 \%$ of the Tile Calorimeter readout channels. In addition, the mean time difference between the $\mathrm{HG}$ and the LG was measured to be $(2.3 \pm 0.4) \mathrm{ns}$. One limitation in the laser system for timing calibration is understanding the propagation time in the laser fibres from the laser source to the PMTs. For this reason, the inter-partition timing and global timing with respect to the rest of ATLAS were coarsely set using cosmic-ray data and more accurately using 2008 beam data.

\footnotetext{
${ }^{10}$ This uncertainty is $(-1.1 \%,+0.7 \%)$ for October 2008 , the period in which the cosmic muon data of this paper were collected.
} 
Fig. 22 Timing of TileCal signals recorded with single beam data in September 2008 (a and b), November 2009 (c) and February 2010 (d). The time is averaged over the full range of the azimuthal angle $\phi$ for all cells with the same

$Z$-coordinate (along beam axis), shown separately for the three radial layers. Corrections for the muon time-of-flight along the $z$ axis are applied in the (b), (c) and (d) figures, but not on the top left (a)
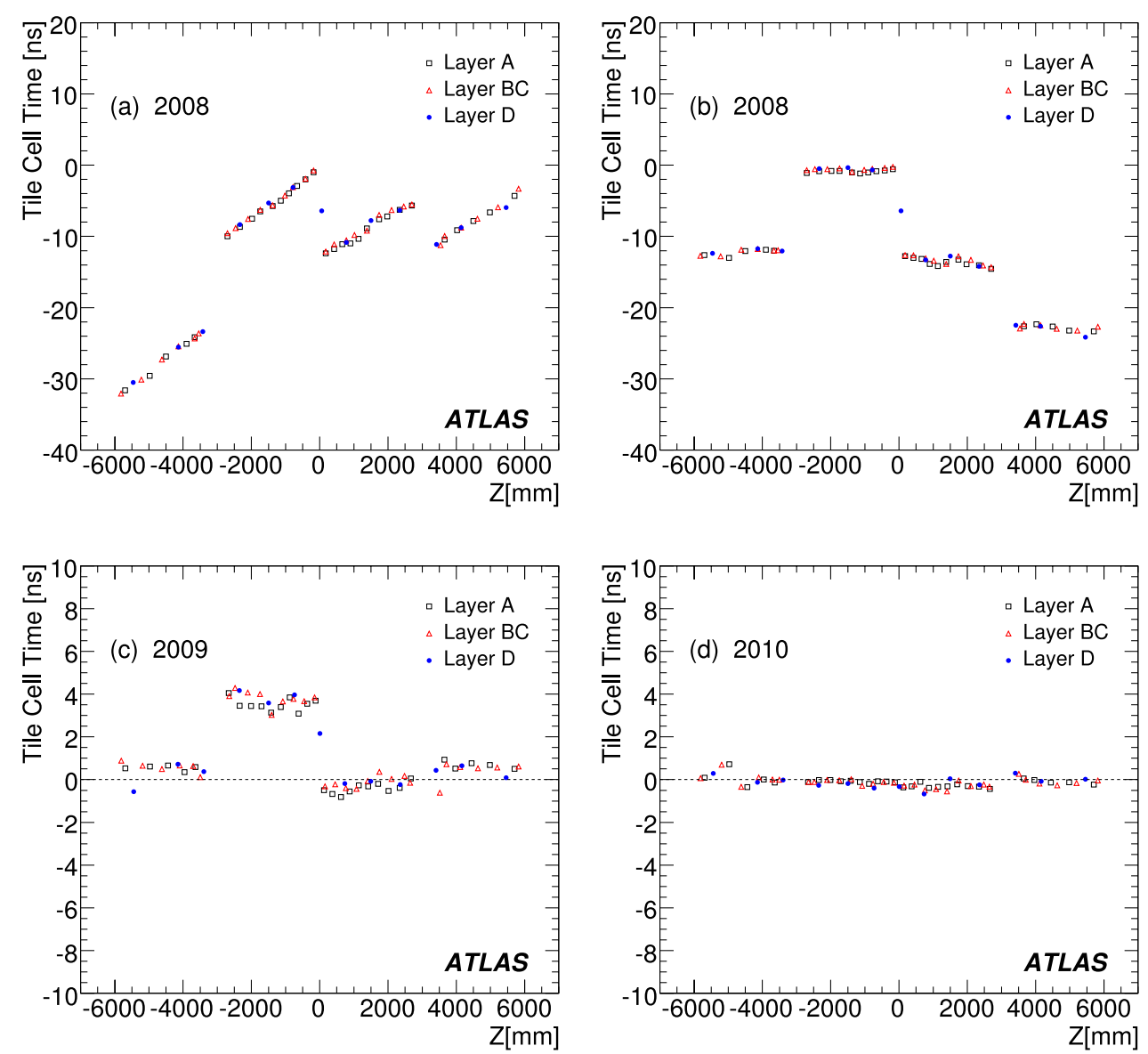

The timing calibration based on laser data was validated using beam splash events. These events contain millions of high-energy particles arriving simultaneously in the ATLAS detector. Since the total deposited energy is large, it is only possible to study the timing response in the LG. Using these events, the time intercalibration of individual channels in the same module was confirmed to be $0.6 \mathrm{~ns}$.

Figure 22 shows the cell time measured in beam splash events, averaged over the full range of the azimuthal angle $\phi$ for all cells with the same $z$-coordinate of ATLAS (along the beam axis). The visible discontinuities at $Z=0$ and $Z= \pm 3000 \mathrm{~mm}$ for the 2008 data are due to the uncorrected time differences between the four TileCal partitions. These were calculated using the 2008 data and adjusted for the 2009 running period. After the muon time-of-flight corrections (b), the timing shows an almost flat distribution within $2 \mathrm{~ns}$ in each partition, confirming a good intercalibration between modules with the laser system. The residual slopes, present in all modules, were corrected for by comparing the 2008 single beam data to the laser data and optimising the effective speed of light in the calibration system optical fibres. Consequently, in 2009, the TOF-corrected timing distribution (c) is even more uniform. In preparation for the 2010 run, the 2009 single beam results were used to pro- vide the offsets for all cells and, as is shown in Fig. 22(d) for the 2010 single beam results, all remaining disuniformities were corrected for. The spread of the TileCal cell timing distribution at the start of the $7 \mathrm{TeV}$ collisions is of $0.5 \mathrm{~ns} .{ }^{11}$

\section{Performance with cosmic ray muons}

The calorimeter response to muons is an important issue since isolated muons will provide a signature of interesting physics events in the LHC collisions phase. For example, semileptonic $t \bar{t}$ decays, the so-called "gold-plated" Higgs decay channel $H \rightarrow Z^{0}+Z^{0}$ and some SUSY processes involve high- $p_{\mathrm{T}}$ muons in their final states, while low- $p_{\mathrm{T}}$ muons originate from $B$-meson decays [20]. In addition, since the interaction of muons with matter is well understood, the prediction of this response is reliable, and its investigation with data can provide information on the detector performance and intercalibration.

The TileCal energy response performance was studied using cosmic muon data collected in 2008 , with the goal of

\footnotetext{
${ }^{11}$ This value takes into account $97 \%$ of the TileCal channels. The timing for the remaining outliers was adjusted offline.
} 
verifying the calibration in terms of EM scale and its uniformity over the whole calorimeter. After an initial comparison of the muon energy signal and the corresponding noise in the same set of cells (in Sect. 5.1), the methods and results of the studies of muon response versus path length are described. These studies were based on the extrapolation into TileCal of cosmic muon tracks reconstructed by the Inner Detector, which is described in Sect. 5.2.2. The performance of the energy response to testbeam muons was also checked at low energy, for comparison.

Muon response results and comparison to Monte Carlo simulations are presented in Sect. 5.3. This Section focuses on several key issues: the response uniformity versus radial layer, $\eta$ and $\phi$, the propagation of the EM scale from testbeam to the full detector configuration in the ATLAS cavern, and a discussion on the systematic uncertainties, such as the ones arising from possible biases of the muon response estimation with the muon momentum and path length. A separate Sect. 5.4 is devoted to calibration of special TileCal cells (ITC, gap and crack scintillators).

The measurement of the time-of-flight of particles in TileCal can be used either for background removal (cosmic and non-collision events) or physics analyses [21]. A good synchronisation of the TileCal cells is important for that, and its validation with cosmic ray muons is described in Sect. 5.5.

\subsection{Muon response compared to noise}

The TileCal readout system is designed so that even small signals induced by muons are well separated from the noise. This feature has been demonstrated with testbeam data [10]. Nevertheless the performance has to be confirmed with data taken with the full ATLAS detector, since the environment is more noisy and changes to the powering system have been made.

This exercise was performed on a large statistics run, with the data sample described in Sect. 2.2: events from various first level triggers were required to have at least one reconstructed Inner Detector track. However, these tracks were not used in any further event or cell selection, for this study. Instead, a different method was used, based on track reconstruction using only TileCal data. This algorithm, named TileMuonFitter, was developed for the data analysis and monitoring of TileCal in the cosmic muon commissioning phase [22, 23]. It uses no external tracking information and uses the set of TileCal cells with energy above a $250 \mathrm{MeV}$ threshold to fit a straight line from the top to the bottom cells (it therefore also ignores the track curvature inside the solenoid magnetic field). In order to reproduce as closely as possible the signal from muons originating in physics collisions, a loose projectivity requirement was imposed. Tracks were selected according to the coordinates of their intersection with the horizontal plane (within $\pm 400 \mathrm{~mm}$ ) and to their angle with respect to the vertical, corresponding to a pseudorapidity range of $0.3<|\eta|<0.4$.

The signal is either the total energy in TileCal summed up over cells selected by the TileMuonFitter algorithm, or the response in the last radial compartment for the D-cells selected by that algorithm. The noise is evaluated from random triggers using the same cells as for signal. The results are shown in Fig. 23 for tracks entering barrel modules within the pseudorapidity range $0.3<|\eta|<0.4$. Top and bottom module responses are considered as two independent entries, so the signal corresponds to that of one module. The signal and noise distributions are well separated for both the total calorimeter response and the last radial layer signal.

In order to estimate the signal-to-noise ratio, the energy distribution is fit to the convolution of a Landau function with a Gaussian. Considering the peak of that convolution fit as the signal, and the RMS of the random trigger distribution as the noise, the signal-to-noise ratio is then $S / N=29$ for the total response and $S / N=16$ for D cells. Since muons are the smallest energy signals that TileCal will measure, these values show a good performance of the calorimeter. The obtained values are lower than for testbeam, ${ }^{12}$ but the difference is consistent with a higher noise level in the ATLAS cavern and with a higher number of cells being summed.

\subsection{Methods for muon response studies}

A brief overview of the analysis methods applied to investigated data samples is provided in this Section. First, we briefly describe the dedicated testbeam (TB) studies with low-energy muons (Sect. 5.2.1). The algorithms and event selection used in the cosmic data analysis are then reported in Sect. 5.2.2.

\subsubsection{Analysis of low energy testbeam muons}

The TB setup, operating conditions and results are summarised in Ref. [10]. Since most of the previous muon TB results were obtained with $180 \mathrm{GeV}$ beams and this energy is too high for the comparison with cosmic ray data, a dedicated study was performed with low-energy muons selected from a pion beam at a nominal energy of $20 \mathrm{GeV}$. These muons originate from pion decay, the distribution of their momenta is calculated to range from $11.5 \mathrm{GeV} / c$ to $20 \mathrm{GeV} / c$, peaking at around $17 \mathrm{GeV} / c$. Data was collected from ten runs with pion beams impinging on one barrel module at different projective incidences, from $-0.65 \leq \eta \leq$ -0.15 and $0.15 \leq \eta \leq 0.45$.

\footnotetext{
${ }^{12}$ In testbeam [10], muon beams at a nominal energy of $180 \mathrm{GeV}$ were used for this study. Taking into account the $20 \mathrm{GeV}$ to $180 \mathrm{GeV}$ response ratio, the testbeam $S / N$ ratios at $20 \mathrm{GeV}$ for the tower and the D cells should amount to 42 and 17 respectively.
} 


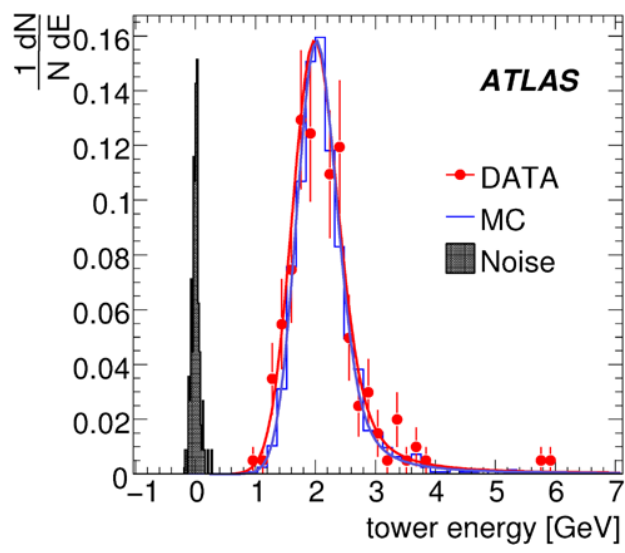

Fig. 23 Example of the muon signal and corresponding noise for projective cosmic muons entering the barrel modules at $0.3<|\eta|<0.4$. Top and bottom modules are treated separately and the momentum range of the cosmic muons was restricted to be between 10 and $30 \mathrm{GeV} / c$. Left: the total energy summed up over selected cells. Right:

Two sets of cuts were applied to select muons from the nominal pion beam:

- Single particle events were selected by requiring a MIPlike response in the beam scintillators upstream of the calorimeter modules. Particles with large angle with respect to the beam axis and/or halo particles were removed by applying suitable cuts in the upstream beam chambers.

- Contrary to muons, pions produce hadronic showers that leave signal also in towers surrounding the one hit by the beam. This feature is exploited in the muon/pion selection-events with signal above noise $\left(E \gtrsim 3 \sigma_{\text {noise }}\right)$ in neighbouring towers were considered pions and were removed from further analysis. Moreover, an upper limit on the response in the impact cell in the first calorimeter radial layer was imposed, in order to avoid pion showers with large electromagnetic shower fraction, whose typical lateral (in $\eta \times \phi$ ) size is smaller than that of a cell.

As the projective beams hit the centre of the given calorimeter tower, the muon response was summed up only from cells in the impact tower. The selection criteria mentioned above guarantee a muon to impinge on the selected tower, therefore no further cut to reject noise events was needed.

The muon track length in the given cell was considered as the radial size of that cell divided by the cosine of the beam incident angle. This approach is fully adequate for projective muons entering the calorimeter at a cell's centre in both $\eta$ and $\phi$ direction, see also Fig. 2.

The Monte Carlo simulation of the TB setup takes into account the detailed detector and beam geometry as well as the momentum distribution of the incident muons.

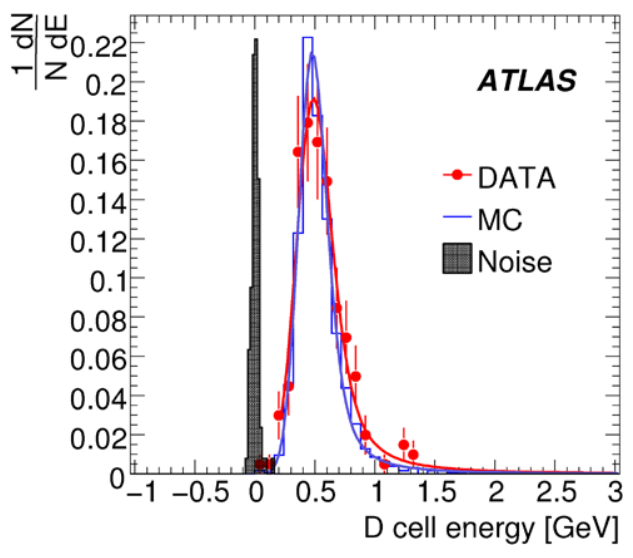

the similar distribution of last radial compartments that can be eventually used to assist in muon identification. The signal (data points with error bars) comes from the cosmic muon data sample (see text), the corresponding noise (filled histogram) is obtained with the random trigger sample

\subsubsection{Analysis of the cosmic ray muons with tracks reconstructed by the Inner Detector}

The performance of the calorimeter was analysed by taking advantage of the information provided by the central tracking. This is an important handle for the study of the calorimeter cell response which is sensitive to the muon path length and momentum.

Track extrapolation and event selection Events were triggered at the first level trigger by RPC and TGC. The tracking information is obtained from the Inner Detector reconstruction, without further contribution from the Muon Spectrometer. Selected events are required to have one reconstructed track in the SCT volume. Events with reconstructed multiple tracks are rejected. Tracks in the TRT do not have $\eta$ information and are not used in the study. The quality of the tracks is enhanced by requiring at least eight hits in the silicon detectors (Pixel and SCT). The tracking requirements introduce some cut-off in the distributions of transverse and longitudinal impact parameters. These are $\left|d_{0}\right| \leq 380 \mathrm{~mm}$ and $\left|z_{0}\right| \leq 800 \mathrm{~mm}$, respectively. ${ }^{13}$

The tracks are extrapolated through the volume of the calorimeters using the tool described in Ref. [24], which uses propagation of the track parameters and covariances that take into account material and magnetic field. Extrapolation is performed in both directions, along the muon momentum and opposite to it. This allows to study the response of modules in the top and bottom part of the detector. Since

\footnotetext{
${ }^{13}$ The transverse impact parameter is defined as the distance to the beam axis of the point of the closest approach of the track to the coordinate origin. The longitudinal impact parameter is the $z$-coordinate (along the beam axis) of the same point.
} 
the track parameters are measured in the centre the method could be sensitive to systematic differences top/bottom.

Figure 24 demonstrates the correct TileCal cell geometry description. It shows the response of cells in the second layer as a function of the $\phi$-coordinate measured at the inner-radius impact point in the given cell. The cells' response average is computed over tracks along the $\eta$ directions in the central barrel region. The responses corresponding to cells of individual modules (width of $\Delta \phi \approx 0.1$ ) are shown with symbols of different colours/styles. The match with the nominal position of the cell edges, displayed by vertical lines, is evident. The total response summed over all modules is superimposed as well and it is reasonably uniform across $\phi$.

The alignment between Tracker and Calorimeter was investigated using tracks with a limited transverse impact parameter $\left(\left|d_{0}\right|<100 \mathrm{~mm}\right)$. The alignment between tracks and nominal cell edges in the second layer of TileCal is within the selected bin size $(\sim 5 \mathrm{~mm})$. This precision is fully adequate for the correct identification of the cells under study and computation of quantities relevant to the analysis.

One of the key parameters of the track is the path length through a given cell. The track extrapolation provides crossing points of the muon track in each radial layer. Additional linear interpolations are performed using the detailed cell geometry to define the entry and exit points for every cell. The track path length is then evaluated as the distance between the entry and exit points for every cell crossed by the muon. In the analysis we consider, for each event, only cells with path length $L>20 \mathrm{~cm}$.

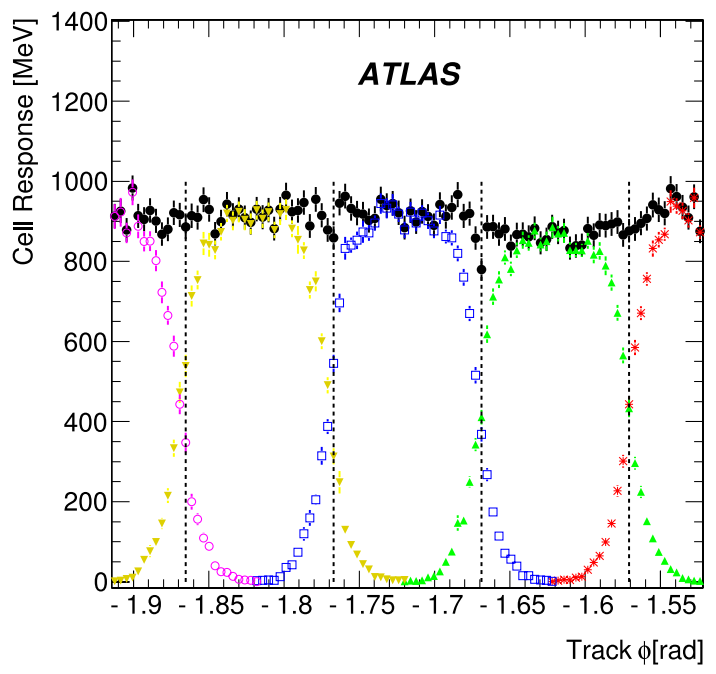

Fig. 24 (Color online) Mean response of cells in the second layer as a function of track $\phi$-coordinate for the bottom central region of the calorimeter. Tracks with $10<p<30 \mathrm{GeV} / c$ were selected. The average response over all central region cells in the given module is shown by symbols of different colours/styles, whereas the total response summed over all modules is shown with black full circles. Vertical lines denote nominal edges of the modules
An upper limit of $30 \mathrm{GeV} / \mathrm{c}$ on the muon momentum is used in the analysis in order to restrict the muon radiative energy losses which show considerable fluctuations and can have an impact on data/MC comparisons. In a small fraction of events the cell response is compatible with the pedestal level although the cells should be hit by a muon. The muon actually hits a neighbouring module. This is consistent with the expected deviation from the muon trajectory due to multiple scattering. In order to limit this effect we restrict the analysis to muons with momenta as measured in the Inner Detector larger than $10 \mathrm{GeV} / c$ and apply a fiducial volume cut requiring the track to be well within the module (that has a half width of $\Delta \phi=0.049$ ):

$\left|\phi_{\text {track }}-\phi_{\text {cell }}\right|<0.045$.

In order to remove residual noise contribution, a cell energy cut of $60 \mathrm{MeV}$ is applied.

Muon tracks close to the vertical direction are badly measured in the Tile Calorimeter due to the strong sampling fraction variation caused by the vertical orientation of the scintillating tiles. To ensure more stable results, tracks are required to enter in the cells with a minimal angle with respect to $\eta=0$ direction. Given the crossing points at the inner and outer cell radial edges we require

$\left|z_{\text {inner }}-z_{\text {outer }}\right| \geq 6 \mathrm{~cm}$.

This cut has an appreciable effect only on very central cells, within the vertical coverage of the ID.

Approximately $100 \mathrm{k}$ data events satisfied the above mentioned selection criteria and were further analysed. The corresponding statistics available in the $\mathrm{MC}$ sample was about twice higher.

Performance checks The track path length is the main handle to study the muon response. Figure 25 shows the response of cells in the second layer as a function of the path length $x$. It includes cosmic events crossing the $\mathrm{BC}$ cells over the entire barrel and over all accepted angles. A clear edge at the path length of $840 \mathrm{~mm}$ is visible in the figure. This represents the radial depth $\Delta R$ of the BC layer cells. Since most cosmic rays are vertical, a large fraction of the muons crossing the central region have a reconstructed path length equal or slightly larger than the layer radius. This is very evident for all cells with a $z$-coordinate within the vertical coverage of the SCT detector $\left|z_{0}\right|<1 \mathrm{~m}$. A linear fit to the corresponding distribution of mean values shows that the muon response scales approximately linearly with the path length, as expected. Figure 25 suggests that the ratio of the cell response with the track path length, i.e. the slope of $\mathrm{d} E / \mathrm{d} x$, is one of the quantities that can be used to study the cell/layers intercalibration. This will be discussed in more detail in Sect. 5.3. 


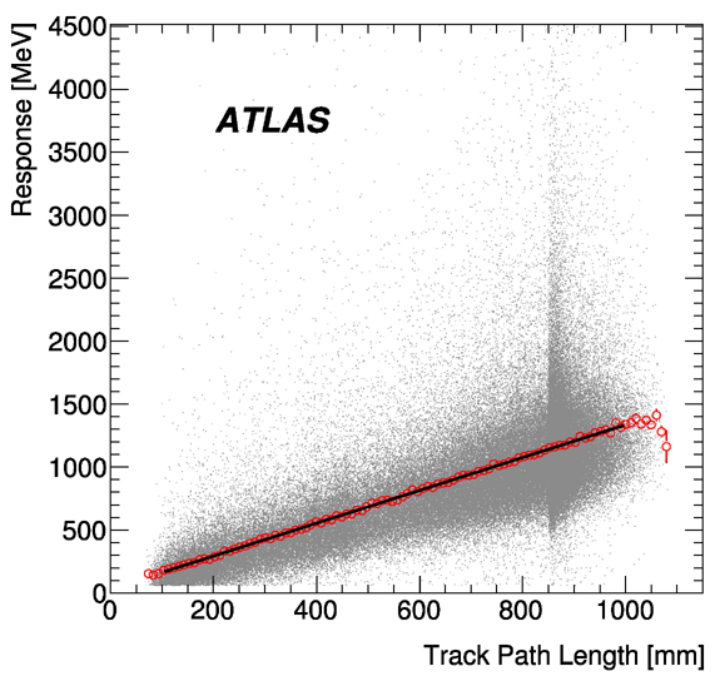

Fig. 25 Mean response of the barrel module BC cells as a function of track path length for tracks with $10<p<30 \mathrm{GeV} / c$. A linear fit to the corresponding distribution of mean values is superimposed. The excess of events at around the track path length of $840 \mathrm{~mm}$ (radial size of the barrel module BC cells) is a purely statistical effect, since most of the cosmic ray muons enter the calorimeter at small zenith angle

\subsection{Performance of energy response}

In this subsection, the results of the calorimeter energy response studies carried out with cosmic muons are reported. The main aim is to cross-check the energy scale set with testbeam and the calibration systems, both in terms of the EM scale and of its uniformity across the detector cells. The uniformity of the response per cell and as a function of pseudorapidity and azimuthal angle is addressed in Sect. 5.3.1, while the layer intercalibration and EM scale issues are discussed in Sects. 5.3.2 and 5.3.3 respectively.

The energy response of TileCal to cosmic muons is probed by estimating the muon energy loss per unit length of detector material, which is obtained by dividing the energy measured by the path length crossed in a given cell (calculated with the method described in Sect. 5.2.2). For simplicity, we call this quantity $\mathrm{d} E / \mathrm{d} x$, even if this is not rigorous, since it is measured in a non-continuous way, and the TileCal cells are made of two different materials, with a direction-dependent sampling fraction.

Our estimator for the muon response is the truncated mean of $\mathrm{d} E / \mathrm{d} x$, defined as the mean in which $1 \%$ of the events in the high-energy tails of the distribution are removed (the number is rounded to the lowest integer). The statistics of the data sample is limited and rare processes like bremsstrahlung or energetic $\delta$-rays can cause large fluctuations of the full mean. The truncated mean is chosen since it is less sensitive to high-energy tails in the cells' response distribution, that are caused by the muon's radiative energy loss. For testbeam, the truncated mean estimator has an additional advantage over the full mean, since it removes resid- ual pion signal contamination. The truncated mean also removes muon events with very large energy deposits (highenergy radiation and/or muon nuclear interactions), therefore the muon/pion selection criterion (see Sect. 5.2.1) does not introduce any bias.

The truncated mean of the energy distribution does not scale linearly with the path length, so there is a small residual dependence of the $\mathrm{d} E / \mathrm{d} x$ on the path length. This is evaluated as a systematic uncertainty and, furthermore, it largely cancels when the ratio of Data/MC is considered.

The dependency of the cell response to the muon momentum was investigated. As can be seen in Fig. 26 (left), the response increases with the momentum as expected, by about $20 \%$ between $p=10 \mathrm{GeV} / c$ and $p=100 \mathrm{GeV} / c$ and there is very good agreement between data and MC from $6 \mathrm{GeV} / c$ to $\sim 100 \mathrm{GeV} / c$. Figure 26 (right) shows that the MC simulations predict a steeper dependence on the muon momentum for the full mean, and some disagreement even for the truncated mean at the higher energies, which could imply some imprecision in the modelling of the muon radiative energy losses.

The real energy loss by muons is typically $10 \%$ lower than the corresponding signal on EM scale and the ratio, known as $e / \mu$, slightly scales with energy [10, 25]. However, in this paper, the validation of the EM scale is carried out by comparing data and Monte Carlo, and response to cosmic and testbeam muons, so this correction factor is not necessary.

\subsubsection{Uniformity of the cell response}

The studies addressed here measure the response uniformity per cell in a layer, as a function of pseudorapidity $\eta$ and azimuthal angle $\phi$ (i.e. per module). Since our estimator is the $1 \%$ truncated mean, we require a minimum of 100 events in each set $-\eta$ or $\phi$ bin, or cell. For the $\eta$ and $\phi$ uniformity analyses, the data is not divided in cells-all cells corresponding to that bin are accumulated and the truncation is applied to the single $\mathrm{d} E / \mathrm{d} x$ distribution for that bin. This approach allows the usage of the largest possible number of cells per bin while minimising biases from fluctuations in the tails. These results comprise all partitions, but exclude the ITC cells (see Sect. 5.4). In addition, we exclude from this study two cells from the D layer with an unusually high $\mathrm{d} E / \mathrm{d} x$.

Muons traverse cells in any direction and at any angle, so the local variations in the optics system (light yield of individual tiles, tile-to-fibre couplings, etc.) are supposed to be averaged out.

Uniformity per cell The uniformity of the cell response is shown in Fig. 27 for each radial layer and the RMS values are summarised in Table 4 . The selection criteria, especially 
Fig. 26 (Left) Muon response $\mathrm{d} E / \mathrm{d} x$ as a function of momentum as measured in the Inner Detector, estimated with the truncated mean for both data and Monte Carlo. (Right) Ratio of Data over Monte Carlo for the muon response $\mathrm{d} E / \mathrm{d} x$ as a function of momentum, shown for the truncated and full mean. For both distributions the D5 cells in the bottom of the extended barrel (A side)

Fig. 27 Distribution of the truncated mean $\mathrm{d} E / \mathrm{d} x$ per cell, shown separately for each radial layer A, BC and D, for data and Monte Carlo. The momentum restricted to be between 10 and $30 \mathrm{GeV} / c$ response is averaged over the range of the cosmic muons was
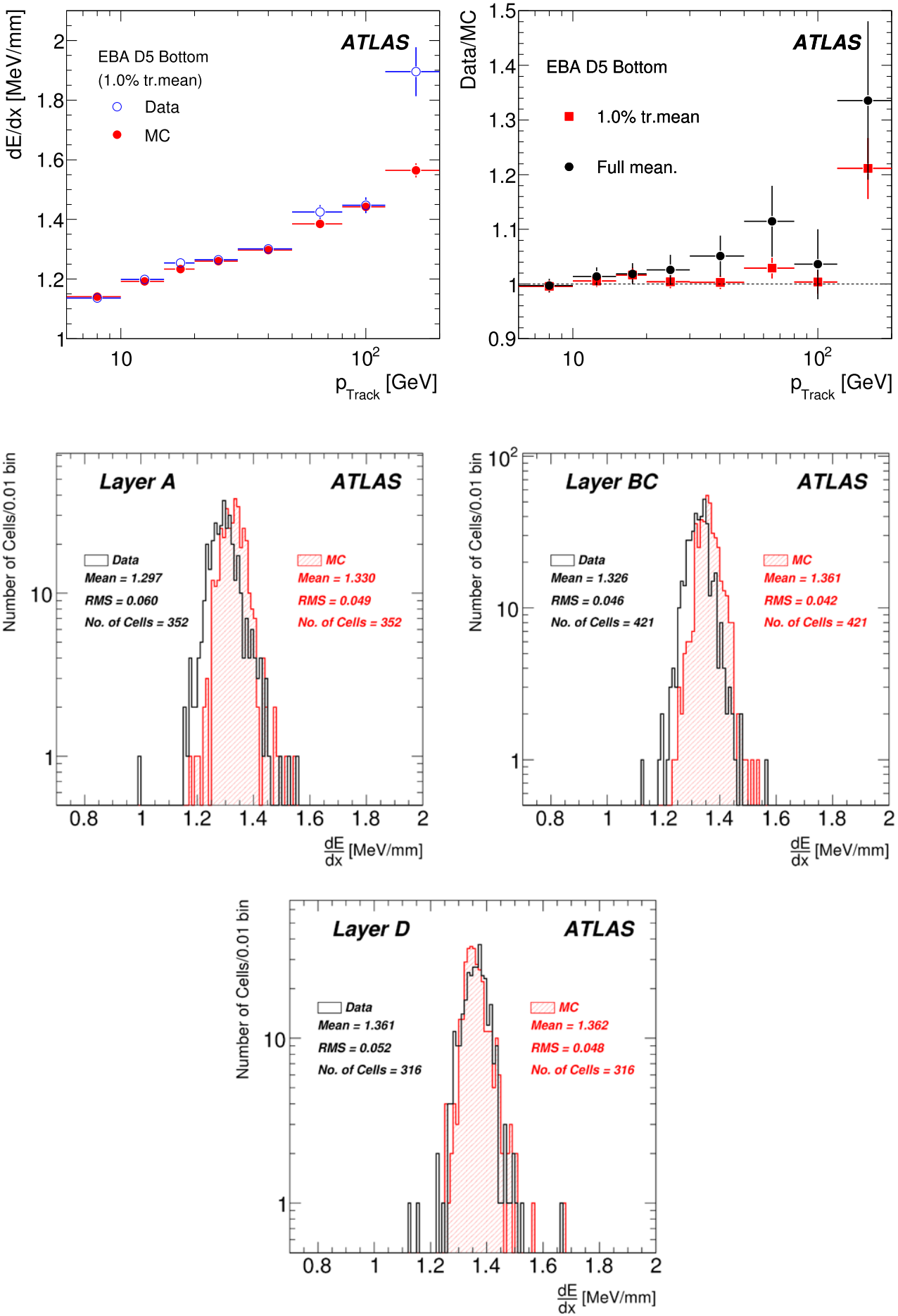

the requirement of 100 events per cell, limit the number of measured cells to the values shown in the figure and table, but still a quite representative fraction of $23 \%$ of the total cells is considered. The statistical population for the simulated and real data used for this study is identical.

The observed spread is the combination of different factors: statistical fluctuations, systematic errors due to the inherent limitations of measuring the cell response with the $\mathrm{d} E / \mathrm{d} x$ of cosmic muons, and the spread in the cell EM scale inter-calibration.

The Monte Carlo simulation has no variation in the quality of the optical components of the calorimeter or in the channel signal shape. Such variations are present in the data but it is difficult to disentangle between the spread due to them or to the statistical fluctuations from an underlying systematic due to the measurement method. Since the MC 
Fig. 28 Momentum of the selected cosmic muon tracks as a function of pseudorapidity $\eta$, for both data and Monte Carlo. No momentum selection is applied in the left side distribution, while on the right, only tracks with momenta within $10 \mathrm{GeV} / c$ and $30 \mathrm{GeV} / c$ are shown. The vertical error bars in the upper part show the RMS of the momentum distribution in each $\eta$ bin; in the lower part the error bars represent the uncertainty on the data/MC value shown
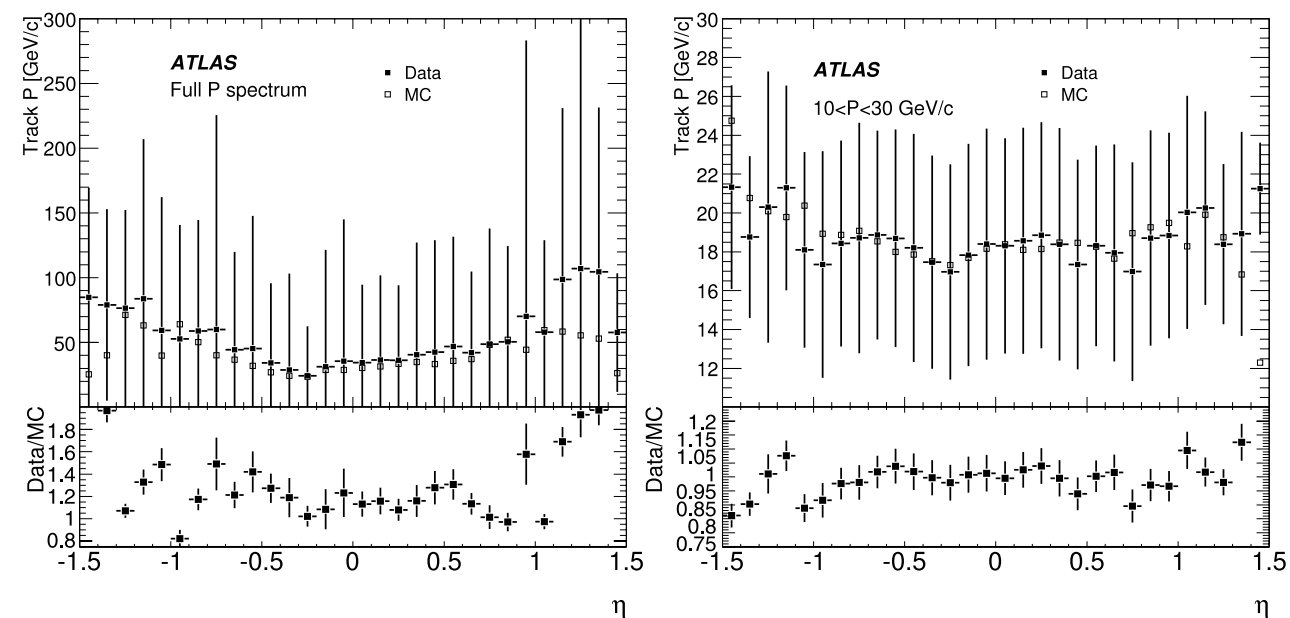

Table 4 The uniformity at the cell level for individual radial compartments. The listed values represent the RMS of the respective distribution of the truncated mean $\mathrm{d} E / \mathrm{d} x$ for that layer, shown for data and Monte Carlo. The number of cells considered, and the fraction of the total that they represent, are also shown

\begin{tabular}{lllll}
\hline Layer & \multirow{2}{*}{$\begin{array}{l}\text { Number of } \\
\text { cells }\end{array}$} & $\begin{array}{l}\text { Fraction of } \\
\text { cells }\end{array}$ & \multicolumn{2}{c}{ RMS $(\mathrm{MeV} / \mathrm{mm})$} \\
\cline { 4 - 5 } & 352 & $18 \%$ & Data & MC \\
\hline A & 352 & $22 \%$ & 0.060 & 0.049 \\
BC & 421 & $38 \%$ & 0.046 & 0.043 \\
D & 316 & & 0.052 & 0.048 \\
\hline
\end{tabular}

shows an RMS in every layer compatible with that of data, it indicates that cells are well intercalibrated within layers.

From the mean of the $\mathrm{d} E / \mathrm{d} x$ distributions per layer it is observed that there is a response discrepancy of $5.0 \%$ between layer A and layer $\mathrm{D}(2.3 \%$ between layer $\mathrm{A}$ and $\mathrm{BC}$ ) for the cosmic muon data, an issue which is further discussed in Sect. 5.3.2.

The variations as a function of pseudorapidity and azimuthal angle, presented in the following paragraphs, were studied separately in each layer, since they appear to be smaller than the dominating inter-layer differences just shown. Another reason is that the cosmic muons are in general non-projective, so most muon tracks cross the calorimeter in each radial layer at different values of $\eta$ and/or $\phi$. Dealing with the total response as a function of $\eta, \phi$ would require projective muons only, thus significantly limiting the available statistics. The results are presented here relative to the average $\mathrm{d} E / \mathrm{d} x$.

Uniformity per pseudorapidity When investigating the uniformity as a function of pseudorapidity, the signal distribution includes all cells with the same azimuthal angle. A possible residual dependency of the muon momentum on the pseudorapidity of the detector cells (that could be due to the access shafts) was investigated. Figure 28 (left) shows that the observed muon momentum distribution is harder than what expected by the Monte Carlo simulation, especially at high values of pseudorapidity. However, in the low momentum region that was selected for the analysis (between 10 and $30 \mathrm{GeV} / c$, see Sect. 5.2.2), the agreement is much better and the variations of momentum with $\eta(\sim 10 \%)$ are quite tolerable for this study.

The tracks identified in the ID are required to point to the cell centre, as specified in (4), as well as the other selection procedures of Sect. 5.2.2. The results are shown in Fig. 29 separately for each radial compartment. It can be seen that, for all layers, the values for the long barrel (central region, $|\eta|<1$ ) are scattered within a $\pm 2 \%$ band around the average. At high $\eta$, in the extended barrel, the statistical uncertainties are larger due to worse coverage than in the central regions. Still these values are for the most part distributed within a $\pm 3 \%$ band.

Uniformity vs. module The uniformity over modules has also been investigated. The response in every module was integrated over all cells in the given radial layer. Studies combine all partitions, barrel and extended barrels.

The results are shown in Fig. 30. Again the same cut on momentum $10<p<30 \mathrm{GeV} / c$ as measured in the Inner Detector was applied. This condition plays two roles-apart from the reason mentioned in Sect. 5.2.2 it also ensures a similar initial momentum distribution in different $\phi$-regions.

Both experimental data and MC exhibit an essentially flat response as a function of azimuthal angle $\phi$. A residual pattern observed with data matches the MC: this small increase of $\mathrm{d} E / \mathrm{d} x$ in horizontal $(\phi \rightarrow 0, \phi \rightarrow \pm \pi)$ modules is likely due to a difference in muon momentum in events passing the selection criteria. Nevertheless, the data show a good uniformity over $\phi$ and, except a few cases in the horizontal region, most modules are well within a $\pm 3 \%$ band. In particular the average response in top $(\phi \approx \pi / 2)$ and bottom $(\phi \approx-\pi / 2)$ modules appears to be within $1 \%$. 
Fig. 29 Uniformity of the cell response to cosmic muons, expressed in terms of normalised truncated mean of $\mathrm{d} E / \mathrm{d} x$, as a function of pseudorapidity $\eta$ for each radial layer. The response is integrated over all cells in each pseudorapidity bin in the given radial layer. The results for data are compared to MC simulations and both are normalised to their averages for each layer. Data are shown with closed circles, open circles indicate MC predictions. Statistical uncertainties only. Horizontal lines limiting a $\pm 3 \%$ band are shown
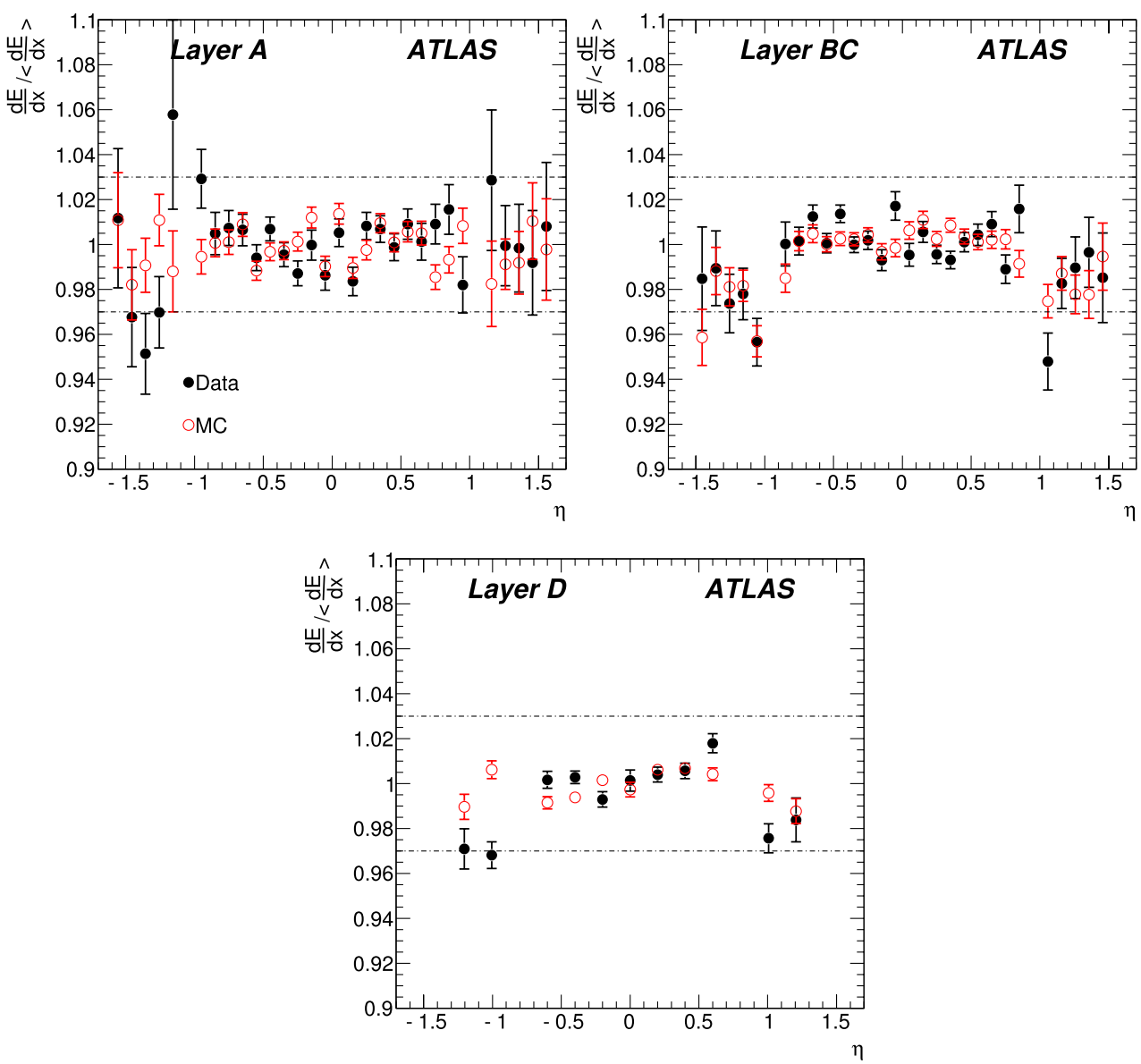

\subsubsection{Muon response and layer intercalibration}

The results discussed in Sect. 5.3.1 show that the cells are reasonably intercalibrated within a given layer, while there are differences observed between individual layers. In order to better quantify these differences, the layer response was calculated as the truncated mean of a single $\mathrm{d} E / \mathrm{d} x$ distribution for all cells in a given layer. This approach reduces the statistical uncertainties, with respect to taking the truncated mean in each cell or $\eta, \phi$ bin. In addition, only events in the bottom of the detector are used, to avoid a bias from the muon momentum cut-in this way, the muon momentum for all the events is measured in the Inner Detector just prior to their incidence in TileCal.

In the cosmic muon analysis, various sources of systematic uncertainties associated with the truncated mean of $\mathrm{d} E / \mathrm{d} x$ have been carefully studied. For every contribution, the associated parameter was varied in the given range and the systematic uncertainty contribution was evaluated as half of the difference between the maximum and minimum resulting truncated mean, unless explicitly stated otherwise.

The following contributions were identified:

- As already shown in Fig. 26 (right), data and MC exhibit a slightly different behaviour in function of the muon mo- mentum. Because of this, the variation of the data/MC ratio over the analysis range $(10-30 \mathrm{GeV} / \mathrm{c})$ is considered as the systematic uncertainty due to the response dependence on the muon momentum.

- As the muon momentum is measured in the Inner Detector located in the centre of ATLAS, the response in the top and bottom part of TileCal can be different. Although the difference is well below $1 \%$ (see also Sect. 5.3.1), we consider its half as the contribution to the systematic uncertainty.

- Another contribution is associated with the residual dependence of the truncated mean on the path length. The truncated mean $\mathrm{d} E / \mathrm{d} x$ was evaluated for several path length bins, and the above mentioned difference was calculated.

- The truncation itself represents another source of systematic uncertainty, that is associated with uncertainties in the description of the energy response shape. The uncertainty was estimated by comparing the resulting truncated mean of $\mathrm{d} E / \mathrm{d} x$ for several values of truncation between $0 \%$ and $2.5 \%$. This contribution does not fully cancel for the Data/MC ratio due to the difference that is observed in the tails of the $\mathrm{d} E / \mathrm{d} x$ distribution between data and MC. 
Fig. 30 Uniformity of the cell response to cosmic muons, expressed in terms of normalised truncated mean of $\mathrm{d} E / \mathrm{d} x$, as a function of azimuthal angle (module) for each radial layer. The response is integrated over all cells in each module in the given radial layer. All partitions are combined. The results for data are compared to MC simulations and both are normalised to their averages for each layer. Data are shown with closed circles, open circles indicate MC predictions. Statistical uncertainties only.

The gap around $\phi=0$ corresponds to horizontal modules that are poorly populated by cosmic ray muons passing through the Inner Detector. Horizontal lines limiting a $\pm 3 \%$ band are shown
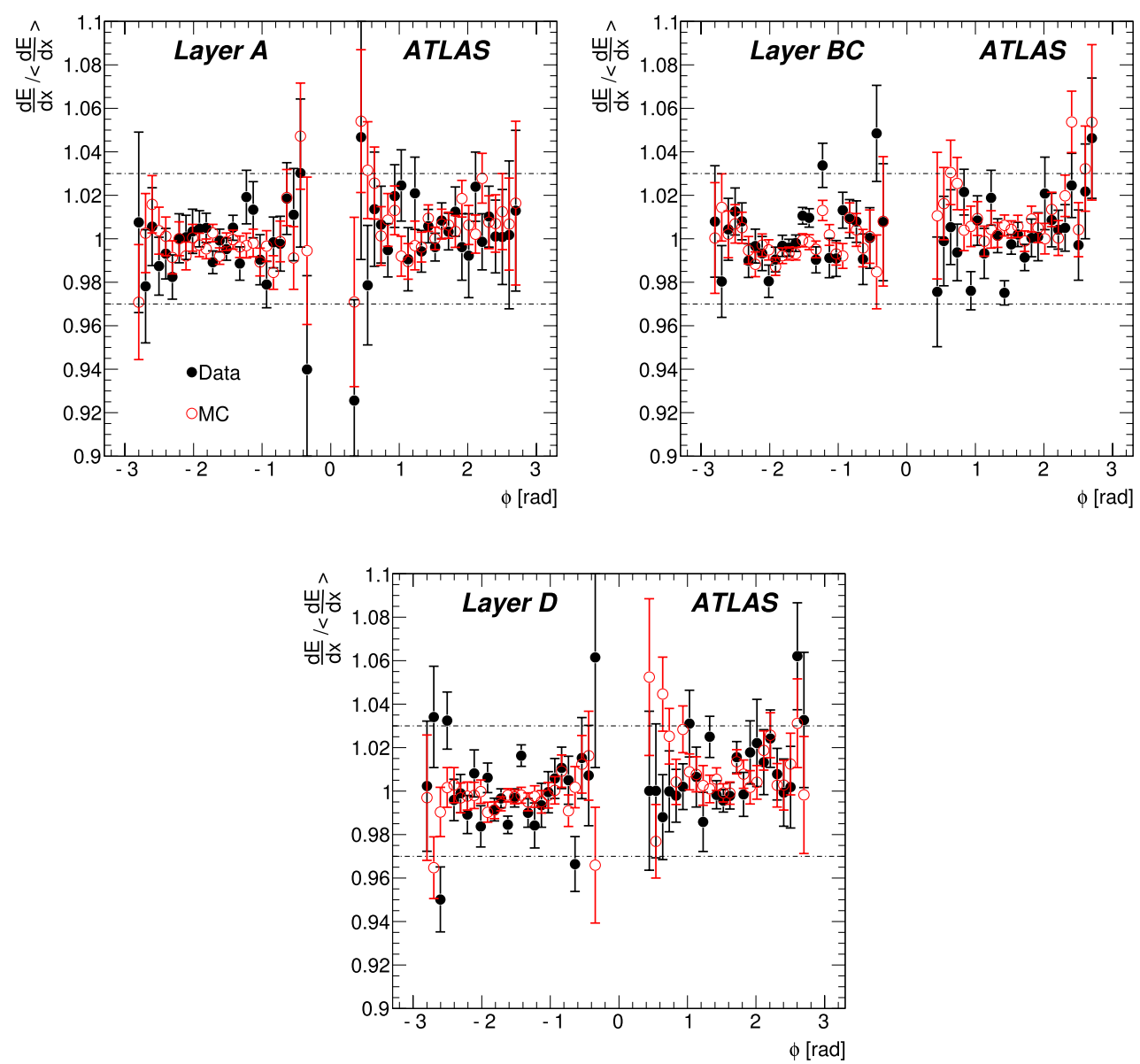

- The impact of the noise cut was studied as well, varying it between $30 \mathrm{MeV}$ and $90 \mathrm{MeV}$ (approximately $1 \sigma$ and $3 \sigma$, where $\sigma$ is the average noise RMS). The associated systematics appears to be very small.

- The measured response was also compared for various triggers, whose efficiencies depend on the muon momentum and also event topology. The data triggered by TGC and RPC indicate a good match within uncertainties, therefore the associated systematics is considered to be negligible with respect to other contributions mentioned above.

- The EM scale was transferred from testbeam to the ATLAS cavern by means of the Cs source calibration procedure. Since the scale was set when the magnetic field was switched off and data were collected with magnetic field on, the appropriate correction has to be applied. Moreover, the Cs data show a response increase with time (see Sect. 4.3). Most of the cosmic data were acquired in September/October 2008, therefore we used the last Cs measurement with magnetic field on before the cosmic data taking to correct for both effects mentioned. The combined effect of these two corrections (magnetic field and response increase) amounts to $-1 \%$ for the barrel and $-0.6 \%$ for the extended barrel between June and Septem-
ber/October 2008 as shown in Fig. 20. Since the origin of the Cs response variation in time is not yet fully understood, the maximum and minimum of the Cs response in 2008 is considered as input for the corresponding asymmetric systematic uncertainty.

The uncertainties were evaluated separately for the LB and EB partitions and per individual radial layer for data, Monte Carlo, and the data/MC ratio (some contributions cancel in the ratio). The correlations among the radial layers are not taken into account and only the square roots of the diagonal terms of the error matrix are considered, and listed in Table 5.

The results on the longitudinal layer intercalibration are presented in Table 6 and displayed in Fig. 31, the error bars representing the total uncertainty based on the quadratic sum of the statistical and systematic uncertainties.

The differences in the cosmic muon response among individual layers are present even after correcting for the residual dependencies on the path length, momentum, impact angle, impact point, by considering the ratio of data over Monte Carlo. The resulting values are strongly correlated, therefore the maximum difference of $4 \%$ between the indi- 
Table 5 The individual contributions to the systematic uncertainty of the truncated mean $\mathrm{d} E / \mathrm{d} x$ in cosmic muon Data and Monte Carlo. The listed values correspond to the diagonal terms of the error matrix.
Analyses were performed with the ID-track method. The uncertainties on the global EM scale factor are discussed in Sect. 4.5

Systematic Uncertainties $[\mathrm{MeV} / \mathrm{mm}]$ for Data and MC

\begin{tabular}{|c|c|c|c|c|c|c|c|}
\hline \multirow{2}{*}{\multicolumn{2}{|c|}{ Uncertainty source }} & \multicolumn{3}{|c|}{ Long Barrel } & \multicolumn{3}{|c|}{ Extended Barrel } \\
\hline & & $\overline{\mathrm{A}}$ & $\mathrm{BC}$ & $\mathrm{D}$ & $\overline{\mathrm{A}}$ & $\mathrm{B}$ & $\mathrm{D}$ \\
\hline \multirow{3}{*}{ Path } & Data & \pm 0.016 & \pm 0.030 & \pm 0.019 & \pm 0.046 & \pm 0.030 & \pm 0.017 \\
\hline & $\mathrm{MC}$ & \pm 0.006 & \pm 0.008 & \pm 0.013 & \pm 0.014 & \pm 0.015 & \pm 0.022 \\
\hline & Data/MC ratio & \pm 0.008 & \pm 0.016 & \pm 0.009 & \pm 0.033 & \pm 0.021 & \pm 0.019 \\
\hline \multirow{3}{*}{ Momentum } & Data & \pm 0.024 & \pm 0.034 & \pm 0.033 & \pm 0.037 & \pm 0.043 & \pm 0.044 \\
\hline & $\mathrm{MC}$ & \pm 0.032 & \pm 0.042 & \pm 0.035 & \pm 0.020 & \pm 0.042 & \pm 0.044 \\
\hline & Data/MC ratio & \pm 0.008 & \pm 0.007 & \pm 0.004 & \pm 0.024 & \pm 0.005 & \pm 0.009 \\
\hline \multirow{3}{*}{ Noise } & Data & \pm 0.007 & \pm 0.002 & \pm 0.002 & \pm 0.009 & \pm 0.004 & \pm 0.003 \\
\hline & MC & \pm 0.004 & \pm 0.002 & \pm 0.003 & \pm 0.003 & \pm 0.002 & \pm 0.002 \\
\hline & Data/MC ratio & \pm 0.002 & \pm 0.000 & \pm 0.001 & \pm 0.005 & \pm 0.001 & \pm 0.000 \\
\hline \multirow{3}{*}{ Truncation } & Data & \pm 0.013 & \pm 0.014 & \pm 0.013 & \pm 0.013 & \pm 0.013 & \pm 0.013 \\
\hline & $\mathrm{MC}$ & \pm 0.014 & \pm 0.014 & \pm 0.014 & \pm 0.014 & \pm 0.014 & \pm 0.014 \\
\hline & Data/MC ratio & \pm 0.004 & \pm 0.005 & \pm 0.005 & \pm 0.003 & \pm 0.001 & \pm 0.001 \\
\hline \multirow{3}{*}{ Top/Bottom } & Data & \pm 0.007 & \pm 0.006 & \pm 0.012 & \pm 0.008 & \pm 0.009 & \pm 0.008 \\
\hline & $\mathrm{MC}$ & \pm 0.015 & \pm 0.014 & \pm 0.014 & \pm 0.016 & \pm 0.037 & \pm 0.006 \\
\hline & Data/MC ratio & \pm 0.006 & \pm 0.014 & \pm 0.002 & \pm 0.006 & \pm 0.021 & \pm 0.010 \\
\hline \multirow{5}{*}{$\begin{array}{l}\text { Global EM } \\
\text { scale factor }\end{array}$} & \multirow{2}{*}{ Data } & +0.005 & +0.005 & +0.005 & +0.000 & +0.000 & +0.000 \\
\hline & & -0.013 & -0.013 & -0.014 & -0.008 & -0.008 & -0.008 \\
\hline & $\mathrm{MC}$ & - & - & - & - & - & - \\
\hline & \multirow{2}{*}{ Data/MC ratio } & +0.004 & +0.004 & +0.004 & +0.000 & +0.000 & +0.000 \\
\hline & & -0.010 & -0.010 & -0.010 & -0.006 & -0.006 & -0.006 \\
\hline \multirow{3}{*}{ Total } & Data & $\begin{array}{r}+0.033 \\
-0.035\end{array}$ & $\begin{array}{l}+0.047 \\
-0.049\end{array}$ & $\begin{array}{l}+0.042 \\
-0.044\end{array}$ & $\begin{array}{l}+0.062 \\
-0.063\end{array}$ & $\begin{array}{l}+0.055 \\
-0.056\end{array}$ & $\begin{array}{l}+0.050 \\
-0.051\end{array}$ \\
\hline & $\mathrm{MC}$ & \pm 0.039 & \pm 0.047 & \pm 0.042 & \pm 0.033 & \pm 0.060 & \pm 0.052 \\
\hline & Data/MC ratio & +0.015 & +0.023 & +0.012 & +0.042 & +0.030 & +0.023 \\
\hline
\end{tabular}

Table 6 The truncated mean of $\mathrm{d} E / \mathrm{d} x(\mathrm{MeV} / \mathrm{mm}$, see text), measured with cosmic ray muons in barrel (LB) and extended barrel (EB), and projective testbeam muons. Results are shown for both data and Monte Carlo as well as for each radial layer. For cosmic ray muons, only modules in the bottom part are used. Total uncertainties are quoted. For cosmic data the statistical component is negligible. The systematic uncertainty corresponds to the diagonal terms of the error matrix

\begin{tabular}{|c|c|c|c|c|}
\hline \multicolumn{2}{|l|}{ Radial layer } & \multirow{2}{*}{$\begin{array}{l}\mathrm{A} \\
1.28_{-0.04}^{+0.03}\end{array}$} & \multirow{2}{*}{$\frac{\mathrm{BC}}{1.32 \pm 0.05}$} & \multirow{2}{*}{$\frac{\mathrm{D}}{1.35 \pm 0.04}$} \\
\hline \multirow{3}{*}{ Cosmic muons, LB } & Data & & & \\
\hline & $\mathrm{MC}$ & $1.32 \pm 0.04$ & $1.35 \pm 0.05$ & $1.34 \pm 0.04$ \\
\hline & Data/MC & $0.97_{-0.02}^{+0.01}$ & $0.98 \pm 0.02$ & $1.01 \pm 0.01$ \\
\hline \multirow{3}{*}{ Cosmic muons, EB } & Data & $1.27 \pm 0.06$ & $1.29 \pm 0.06$ & $1.32 \pm 0.05$ \\
\hline & $\mathrm{MC}$ & $1.31 \pm 0.03$ & $1.32 \pm 0.06$ & $1.34 \pm 0.05$ \\
\hline & Data/MC & $0.97 \pm 0.04$ & $0.98 \pm 0.03$ & $0.99 \pm 0.02$ \\
\hline \multirow{3}{*}{ Testbeam, LB } & Data & $1.25 \pm 0.03$ & $1.39 \pm 0.04$ & $1.39 \pm 0.03$ \\
\hline & $\mathrm{MC}$ & $1.30 \pm 0.02$ & $1.37 \pm 0.03$ & $1.36 \pm 0.02$ \\
\hline & Data/MC & $0.96 \pm 0.02$ & $1.02 \pm 0.04$ & $1.02 \pm 0.02$ \\
\hline \multicolumn{2}{|c|}{ Double ratio $\frac{(\text { Data/MC })_{\text {Cosmic muons, } \mathrm{LB}}}{(\text { Data/MC })_{\mathrm{TB}, \mathrm{LB}}}$} & $1.01 \pm 0.03$ & $0.96 \pm 0.04$ & $0.98 \pm 0.03$ \\
\hline
\end{tabular}


vidual measurements with the cosmic muon data indicates the layer response discrepancy.

\subsubsection{Validation of the EM scale propagation from testbeam}

The ratio data/MC mentioned above also depends on the absolute EM scale of the MC simulated energy loss in the calorimeter. Due to the uncertainties in this quantity, the double ratio of data/MC, cosmic muon/TB, is adopted for comparison of the muon response and hence the EM scale between cosmic and TB data in the long barrel. For testbeam data and Monte Carlo, the truncated mean of the $\mathrm{d} E / \mathrm{d} x$ distribution was obtained for each run, and then averaged over all runs. These are the values already presented in Table 6 and Fig. 31. The evaluation of the systematic uncertainties is briefly described below.

We consider the spread of the $\mathrm{d} E / \mathrm{d} x$ values over the different incidence angles as the main uncertainty of the measurement, an approach that effectively combines the statistical and part of systematic uncertainties. On top of them, we consider the following subdominant contributions:

- The bias due to the truncation in the $\mathrm{d} E / \mathrm{d} x$ distribution was estimated in the same way as for cosmic data (mentioned above).

- The uncertainty in the global EM scale due to the noncalibrated integrators (see Sects. 4.3 and 4.4) at that time. This uncertainty applies only to data, not to Monte Carlo.

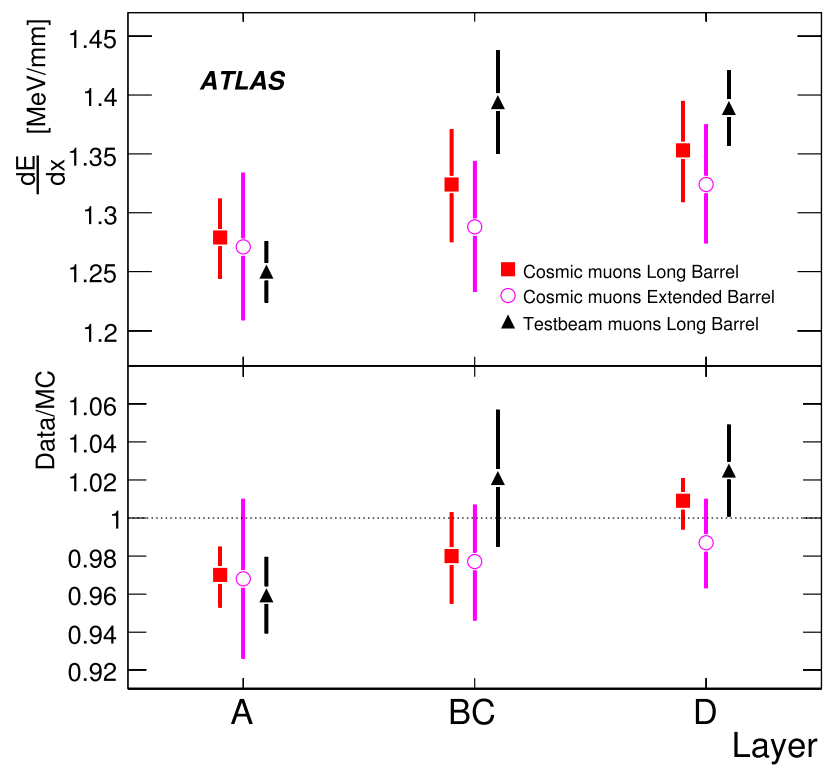

Fig. 31 The truncated mean of the $\mathrm{d} E / \mathrm{d} x$ for cosmic and testbeam muons shown per radial compartment and, at the bottom, compared to Monte Carlo. For the cosmic muon data, the results were obtained for modules at the bottom part of the calorimeter. The error bars shown combine in quadrature both the statistical and the systematic uncertainties, considering only the diagonal terms of the error matrix
The individual uncertainties were evaluated for each radial layer and the resulting total uncertainties, shown in Table 6 , were obtained by summing the individual contributions in quadrature.

The double ratio of data/MC, cosmic muons/TB, is presented in the last row of Table 6 . The uncertainty contributions are computed by propagating in quadrature the TB uncertainties just described and the cosmic muon uncertainties mentioned in the previous section, that only take into account the error matrix diagonal terms. The EM scale measured with cosmic muons, relative to that determined at testbeam in the long barrel, amounts to $1.01,0.96$ and 0.98 for the A, BC and D layers respectively. Since the uncertainties per layer are at most $4 \%$, these values are consistent with 1.0 , showing that, within the precision limits of the analysis, the propagation of the EM scale from testbeam to ATLAS was performed successfully.

It should be noted that the LHC collisions will provide extra tools to check the EM scale calibration. Isolated muons and single hadrons developing their shower only in TileCal will provide two data samples for which a direct comparison to the testbeam scale will be possible.

\subsection{ITC and gap/crack scintillator calibration}

Understanding the response of the intermediate Tile Calorimeter (ITC) and the gap and crack scintillators (see Sect. 2.1 and Fig. 2) to cosmic ray muons is essential for their calibration. The gap and crack scintillators can not be calibrated using the Cs calibration source and therefore have arbitrary calibration factors applied to them. This study with cosmic muons gives the first clues for their in-situ performance.

These detectors are calibrated in two steps. The first step is the intercalibration in $\phi$ among the cells of the same detector type and to determine the calibration factors for each cell. The second step is the absolute calibration and to determine a scale factor defined relative to the MC for each detector type. Since the absolute energy scale in the scintillators is not known, the simulation is used as a reference in this case.

The event selection follows the same procedures as indicated in Sect. 5.2.2, with the exception that only events with a single muon track with a momentum above $5 \mathrm{GeV}$ are considered and that, for the ITC cells, the entry and exit points of the track in the cell must be separated by at least $4 \mathrm{~cm}$ in the $z$ direction. These requirements accept $8 \%$ of RPC triggered events, $80 \%$ of TGC triggered events and $7 \%$ of L1 calorimeter triggered events. Problematic cells and scintillators ${ }^{14}$ are excluded in this analysis.

The geometrical path length is defined as a straight line between the two surfaces of the cell or scintillator. The muon

\footnotetext{
${ }^{14}$ Cells or scintillators that, even though matched with extrapolated tracks, appear too noisy or show very small signal.
} 
energy loss per unit path length is used to evaluate the response. It is referred to as $\mathrm{d} E / \mathrm{d} x$ for the ITC cells (C10, D4), which have the same elementary structure as ordinary TileCal cells (as in Sect. 5.3). For the gap and crack scintillators (E1-E4), the muon energy loss estimator is the signal (expressed in units of charge) normalised to the muon path length through the scintillator and, for distinction, it is referred to as $\Delta E / \Delta L$. Figure 32 is an example of the $\mathrm{d} E / \mathrm{d} x$ or $\Delta E / \Delta L$ distribution for the cells in one module for cosmic ray data and MC. The cells generally show good signal-to-noise separation except for crack scintillators (E3, E4). The signals in the crack scintillators are found to be too small for good separation from noise distributions and the $\mathrm{HV}$ of the PMT has been accordingly increased. The noise distribution in the gap scintillators (E1, E2) in the data is mainly due to grooves and holes in these scintillators that accommodate the ${ }^{137} \mathrm{Cs}$ source pipes.

For each cell (scintillator), the $\mathrm{d} E / \mathrm{d} x(\Delta E / \Delta L)$ distributions were fitted with the convolution of a Landau function with a Gaussian. The average and the RMS of the peak positions (MOP) of the fitted functions are summarised in Fig. 33 and shown with the results from the MC. For comparison, results for the extended barrel cells D5 and B11 are also shown with ITC cells in the figure. Cells with insufficient statistics or with poor fits are excluded and $30 \%-50 \%$ of ITC cells and $\sim 25 \%$ of gap scintillators remain.

The average values indicate that the response for the ITC cells is consistent with the cell response of ordinary TileCal cells, which are well calibrated with the standard Tile Calorimeter calibration procedure. The response of the ITC cells is also consistent with $\mathrm{MC}$ to within $\sim 5 \%$. In the gap scintillators (E1, E2), where the scale is arbitrary, the observed differences of roughly $20 \%$ imply an additional scale factor to adjust data relative to $\mathrm{MC}$.

The uniformity of the response was also determined with these data. The RMS values are $\sim 10 \%$ in ITC cells (C10 and D4), while in gap scintillators (E1, E2) the RMS values amount to $15 \%-20 \%$.

Based on this study, no changes were made to the ITC cells since their response is consistent with the response of the ordinary Tile cells. For the gap scintillators, correction factors for $\phi$ intercalibration and global scale factors were measured relative to MC. As a result of this analysis, the $\mathrm{HV}$ values for the crack scintillators (E3, E4) have been increased to improve the separation between signal and noise. The expected improvement has been verified.
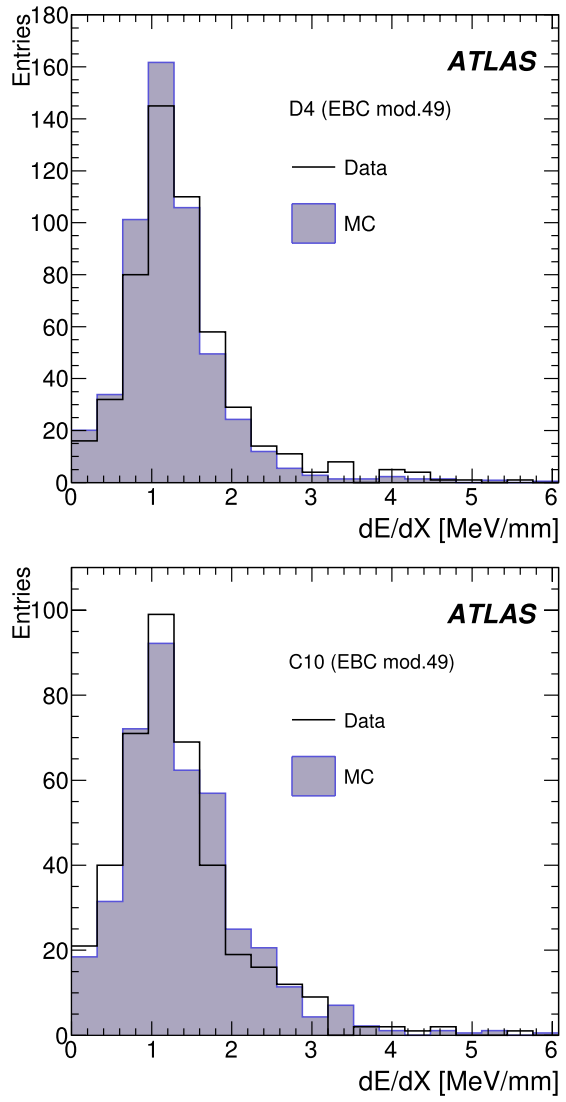
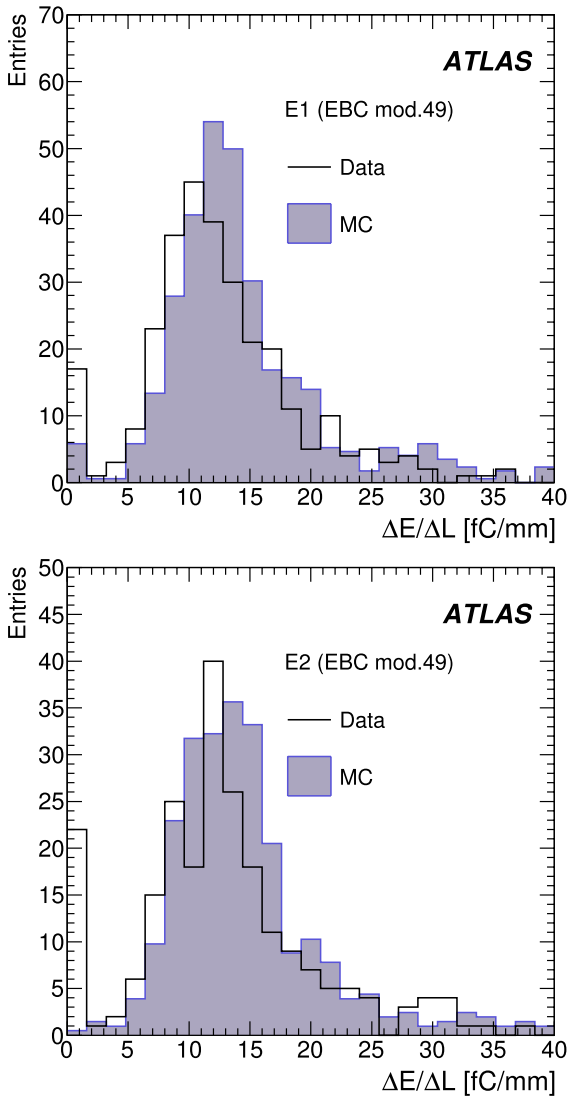
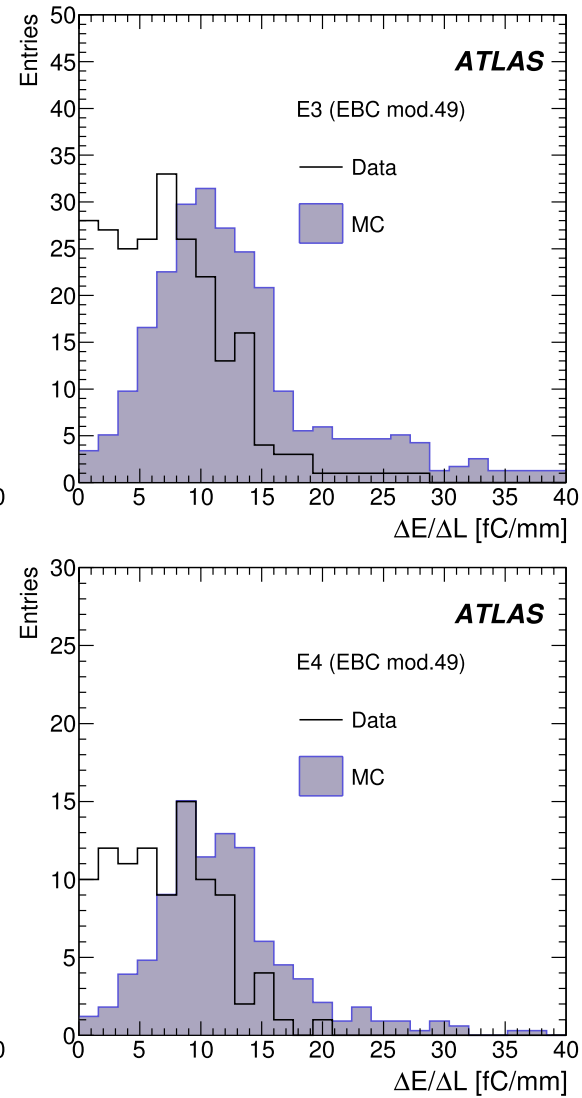

Fig. 32 Responses of ITC cells (D4 and C10), gap scintillator cells (E1 and E2) and crack scintillator cells (E3 and E4) to cosmic ray muons in EBC module 49. They are shown in terms of $\mathrm{d} E / \mathrm{d} x$ for the ITC cells and $\Delta E / \Delta L$ for the gap and crack scintillators 
Fig. 33 Responses of gap and crack scintillators (left) and ITC cells (right) to cosmic muons. Shown are the average values of the peak positions (MOP) of the fitted functions on the $\Delta E / \Delta L$ and $\mathrm{d} E / \mathrm{d} x$ distributions respectively. The vertical bars indicate the RMS values
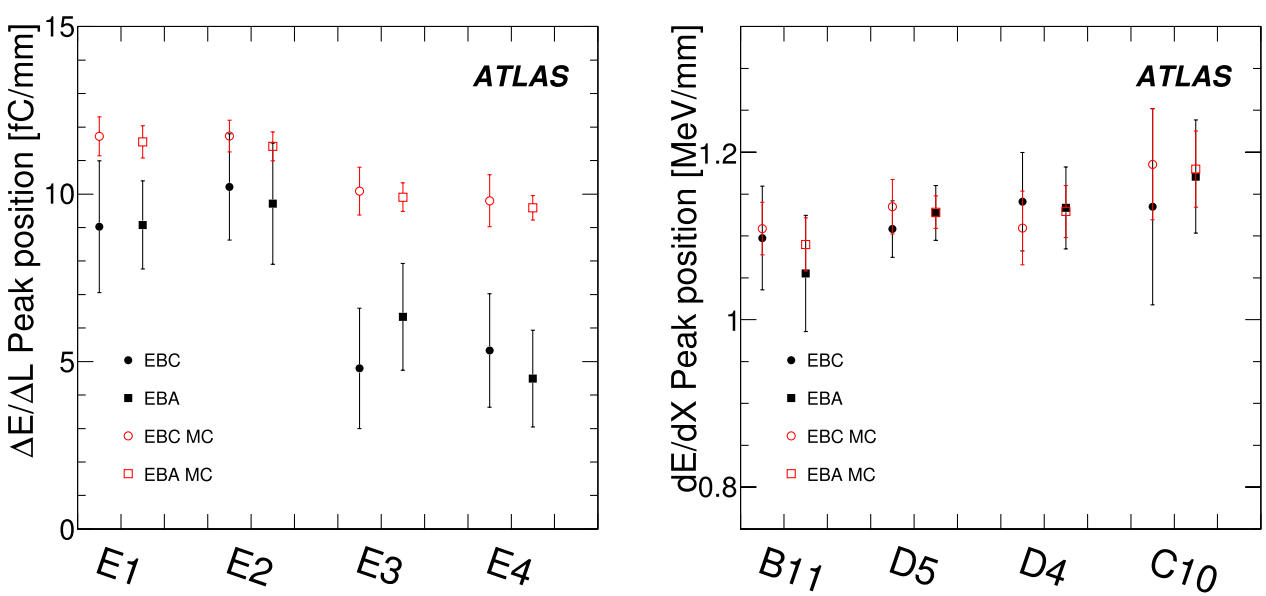

\subsection{Performance of time response}

Before the start of the LHC in September 2008, cosmic muons provided the only way to verify the accuracy of the time calibration of TileCal at the cell level. In addition to the online monitoring of detector synchronisation, that used distributions of average event time in function of position, detailed analyses of the data, described in this section, were able to measure the timing corrections for a large fraction of the TileCal channels. These analyses, based on the measurement of the muon time-of-flight between the top and bottom cells, have been validated using the data from the 2008 LHC single beam.

\subsubsection{Extraction of time corrections}

Two methods have been developed to extract the time corrections using the cosmic data $[26,27]$. They are based on the comparison of the time determined in the top and bottom modules with the time-of-flight of the cosmic muon through the detector.

The iterative method [26] was successfully applied during the 2007 data takings. The very top barrel module (LBA16) was taken as a reference and the time offsets of the other modules (taken as single values for a whole module) were measured relative to this one. Since not all modules can be directly calibrated with respect to the reference one, an iterative procedure has been adopted, determining first the time of modules in the bottom sector opposite to the reference. In subsequent steps, the time of other modules in the top was determined relatively to those in the bottom already measured in the first step, and so on until all modules were analysed. The results of this method showed at an early stage that the laser-based inter-module time offsets had an accuracy of about $\pm 2 \mathrm{~ns}$. The systematic uncertainty due to the method itself was studied by adding known offsets to the input data, and determined to be $0.5 \mathrm{~ns}$. In principle this method could also be used at the cell level, but for this a different method was used.

The global matrix method [27] obtains the timing offsets also from comparison of data from top and bottom of the detector, but does that in an integrated way, by solving a system of equations that relates the time offsets of each cell to the measured time differences between those cells. If $m$ and $n$ are, respectively, the numbers of selected cells in the top and bottom part of the detector, and $k$ is the number of valid pairs (see selection criteria in next paragraph) between them, the problem can be posed in matrix form as:

$M t=\Delta T$

in which $t$ is the $(m+n)$-size vector of unknown offsets, $\Delta T$ is the $k$-size vector of measured time differences (averaged over all events, and corrected for time-of-flight). $M$ is a $(m+n) \times k$ matrix, and each line (of $k$ ) contains 1 for the element of the top part and -1 for the each element of the bottom part corresponding to the pair identified by that line. In order to properly weigh the results for different pairs, each element in $M$ and $\Delta T$ are divided by the standard deviation of the pair time difference measurement. Since $k>(m+n)$, this system of equations is overdetermined, so the (approximate) solution is the least-squares minimum of $\|M t-\Delta T\|$.

This method was applied to $0.5 \mathrm{M}$ events from the RPC trigger sample of a long run taken in 2008. The event selection required to have at least one energy deposit above $250 \mathrm{MeV}$ both on the top and bottom cells. For each event, cells were selected by requiring an energy between 200 $\mathrm{MeV}$ and $20 \mathrm{GeV}$, and a time difference between both PMTs of less than 6 ns. A final selection required that at least 5 events contribute to a cell pair average, and that the RMS of the measurements is smaller than $5 \mathrm{~ns}$. The efficiency of these selections is of $40 \%, 75 \%$ and $82 \%$ for, respectively, the A, BC and D cells. To avoid memory limitations due to the large number of pairs (more than $30 \mathrm{k}$ ), the offset extraction was carried out separately for four sets of pairs. To 
Fig. 34 (Left) Average of the time corrections per module as measured with the global matrix all cells. (Right) Difference of those values with respect to the results from the 2008 single beam data, removing the cells from the first layer. Different symbols correspond to modules in different partitions, as method with cosmic muons, for indicated
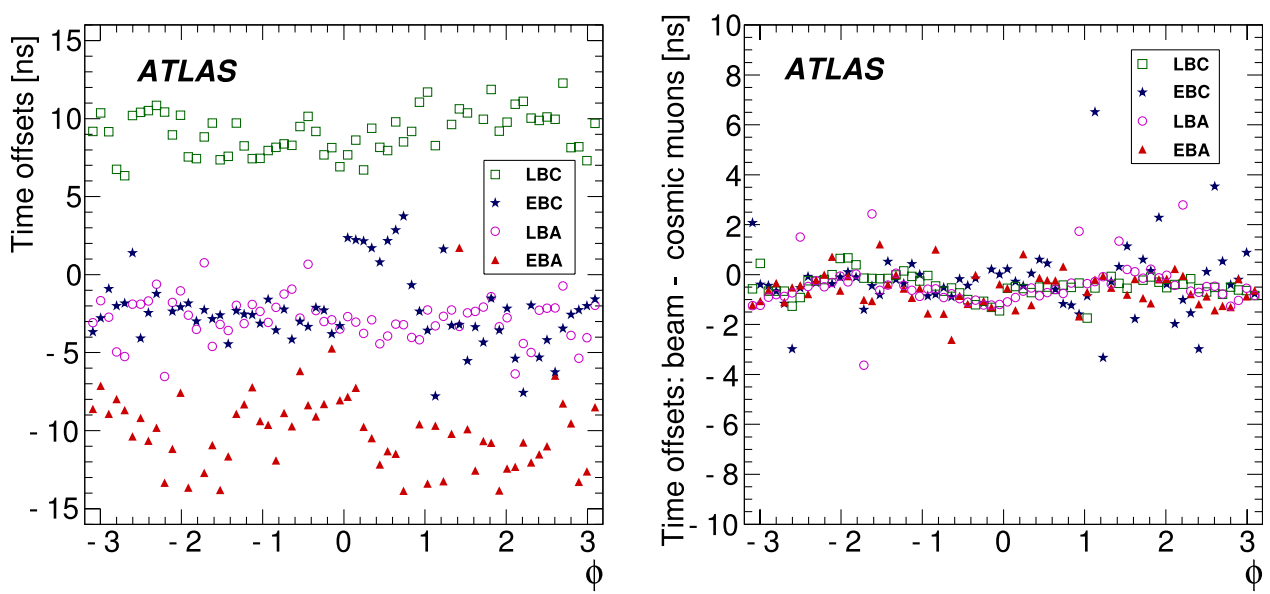

Fig. 35 Correlation (left) and difference (right) between the time corrections from cosmic muons and the 2008 single beam results. The cells from the first radial layer were removed
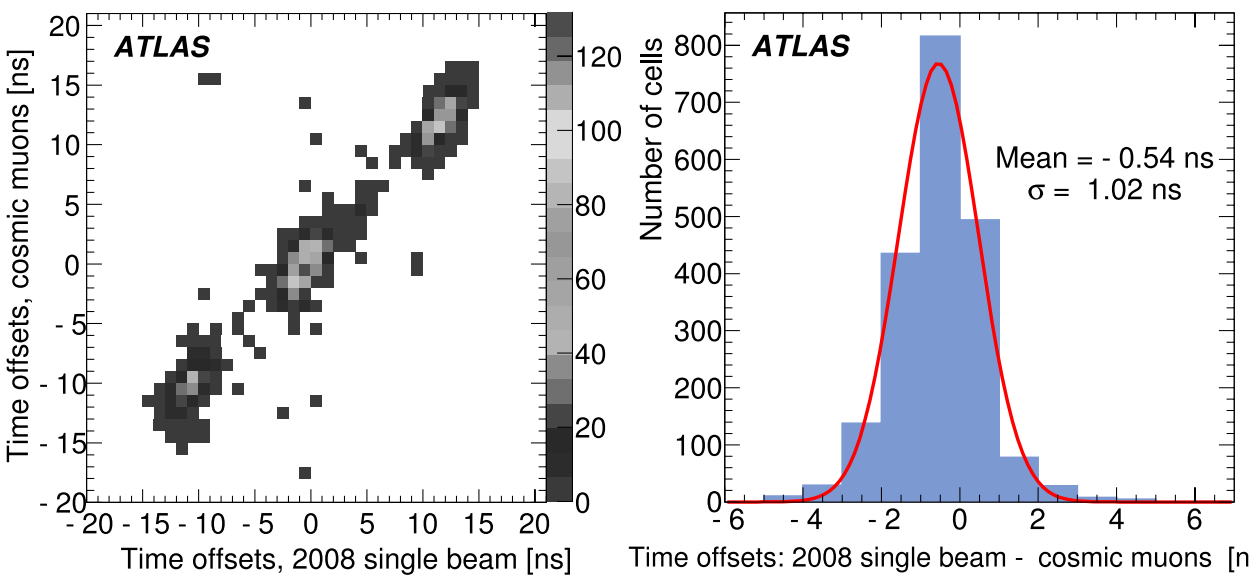

Time offsets: 2008 single beam - cosmic muons [ns] ensure consistency, these sets have a partial overlap, and the results are integrated at the end. The results were compared with those obtained with the 2008 single beam data (see Sect. 4.6), which were taken very close in time (less than 1 month) to the cosmic muon run analysed.

\subsubsection{Results and comparison with 2008 LHC single beam}

The average for each module of the cell offsets measured with the global matrix method is shown in Fig. 34 (left) and the comparison with the single beam data is shown in Figs. 34 (right) and 35.

The results clearly show differences of 10 ns between each partition (Fig. 34 left), but an otherwise good uniformity, of $2 \mathrm{~ns}$, for all the cells in the second and third radial layers within each partition (Fig. 34 right). The results for the first layer are more scattered (this is reflected in the module average distributions, in particular for the EBA partition), in disagreement with the single beam measurements (see also Sect. 4.6). Due to the small size of the cells, the energy deposition with cosmic muons in this layer is small (peaking at roughly half of the value for the second layer), and consequently the signal-to-noise ratio is worse. Since the single beam energy deposition is significantly larger, those results are more reliable, and so only the cosmic muon results from the second and third layers are considered valid.

It was expected to have differences between partitions, since the laser calibration had not been performed at this level. ${ }^{15}$ The difference of $5-8 \mathrm{~ns}$ for the first 8 modules of EBC (Fig. 34 left, between 0 and 0.8 in $\phi$ ) was unexpected, but confirmed with single beam data, and traced to an incorrect measurement of laser fibre lengths. So the inter-partition and inter-module results confirmed and validated the results from single beam, which were subsequently used to set the calibration time offsets, as described in Sect. 4.6. Within each partition, the agreement with the single beam data for the second and third layers, both at the level of module averages and single cells, is about $1 \mathrm{~ns}$. Since this is smaller than the spread of the average offsets, these results provide a measurement of the accuracy of the laser-based time calibration, of about $2 \mathrm{~ns}$.

\footnotetext{
${ }^{15}$ This is because the laser calibration data was taken in Tile standalone configuration, which has different delays than the global ATLAS online configuration.
} 


\section{Conclusions}

The Tile hadronic calorimeter of the ATLAS detector underwent extensive testing during its commissioning and cosmic muon data-taking periods. The calorimeter has $99.1 \%$ (December 2009) of its cells operational and conditions that can affect the PMT gains have been monitored to be very stable over one year, such that no corrections are needed. The noise, being within the expectations and requirements, has a non-Gaussian component which has been taken into account in the reconstruction of clusters and physics objects. The noise magnitude has been stable over time within $1 \%$.

The electromagnetic energy scale has been transferred from $11 \%$ of modules calibrated at testbeam to the full Tile Calorimeter in the ATLAS cavern setting by means of the TileCal calibration systems. The precision of all calibration systems is remarkable and has proven to follow the systems' design requirements. Regular calibration data-taking has demonstrated the stability of individual systems at levels well below $1 \%$.

The single beam data proved to be very useful in complementing the calibration systems for the synchronisation of the calorimeter cells. The timing intercalibration capability is at the level of $1 \mathrm{~ns}$ within a TileCal module and $2 \mathrm{~ns}$ within a partition. Cosmic muons provided an independent cross-check of the time calibration settings, having verified a large fraction of the second and third layer cells with $2 \mathrm{~ns}$ precision.

The analysis of the cosmic muon data has been a very useful validation procedure to assess the performance with particles at the full calorimeter scale and to compare with Monte Carlo expectations. The separation between signal and noise is very good, with an S/N ratio of $\sim 29$ for the sum of the three radial layers. The cell response uniformity, as measured with the muon track $\mathrm{d} E / \mathrm{d} x$, is at the level of $4.6 \%, 3.5 \%$ and $3.8 \%$ within, respectively, the $\mathrm{A}, \mathrm{BC}$ and $\mathrm{D}$ layers. The energy response shows a maximum difference among the radial layers of $4 \%$.

The estimator of the EM scale relative to the testbeam calibration period as determined by the cosmic muons analysis is consistent with 1 , with an uncertainty of $4 \%$. A possible bias of $-5 \%$ in the EM scale calibration due to lower HV settings as compared to the testbeam cannot therefore be totally excluded. However, the measurements with cosmic ray muons are compatible with a successful propagation of the EM scale factor from testbeam to the full ATLAS configuration.

Acknowledgements We are greatly indebted to all CERN's departments and to the LHC project for their immense efforts not only in building the LHC, but also for their direct contributions to the construction and installation of the ATLAS detector and its infrastructure. We acknowledge equally warmly all our technical colleagues in the collaborating Institutions without whom the ATLAS detector could not have been built. Furthermore we are grateful to all the funding agencies which supported generously the construction and the commissioning of the ATLAS detector and also provided the computing infrastructure.

The ATLAS detector design and construction has taken about fifteen years, and our thoughts are with all our colleagues who sadly could not see its final realisation.

We acknowledge the support of ANPCyT, Argentina; Yerevan Physics Institute, Armenia; ARC and DEST, Australia; Bundesministerium für Wissenschaft und Forschung, Austria; National Academy of Sciences of Azerbaijan; State Committee on Science \& Technologies of the Republic of Belarus; CNPq and FINEP, Brazil; NSERC, NRC, and CFI, Canada; CERN; CONICYT, Chile; NSFC, China; COLCIENCIAS, Colombia; Ministry of Education, Youth and Sports of the Czech Republic, Ministry of Industry and Trade of the Czech Republic, and Committee for Collaboration of the Czech Republic with CERN; Danish Natural Science Research Council and the Lundbeck Foundation; European Commission, through the ARTEMIS Research Training Network; IN2P3-CNRS and CEA-DSM/IRFU, France; Georgian Academy of Sciences; BMBF, DFG, HGF and MPG, Germany; Ministry of Education and Religion, through the EPEAEK program PYTHAGORAS II and GSRT, Greece; ISF, MINERVA, GIF, DIP, and Benoziyo Center, Israel; INFN, Italy; MEXT, Japan; CNRST, Morocco; FOM and NWO, Netherlands; The Research Council of Norway; Ministry of Science and Higher Education, Poland; FCT cofinanced by QREN/COMPETE of European Union ERDF fund, Portugal; Ministry of Education and Research, Romania; Ministry of Education and Science of the Russian Federation and State Atomic Energy Corporation ROSATOM; JINR; Ministry of Science, Serbia; Department of International Science and Technology Cooperation, Ministry of Education of the Slovak Republic; Slovenian Research Agency, Ministry of Higher Education, Science and Technology, Slovenia; Ministerio de Educación y Ciencia, Spain; The Swedish Research Council, The Knut and Alice Wallenberg Foundation, Sweden; State Secretariat for Education and Science, Swiss National Science Foundation, and Cantons of Bern and Geneva, Switzerland; National Science Council, Taiwan; TAEK, Turkey; The Science and Technology Facilities Council and The Leverhulme Trust, United Kingdom; DOE and NSF, United States of America.

Open Access This article is distributed under the terms of the Creative Commons Attribution Noncommercial License which permits any noncommercial use, distribution, and reproduction in any medium, provided the original author(s) and source are credited.

\section{References}

1. F. Ariztizabal et al. (TileCal Collaboration) Construction and performance of an iron-scintillator hadron calorimeter with longitudinal tile configuration. Nucl. Instrum. Methods A 349, 384-397 (1994). http://cdsweb.cern.ch/record/262630

2. G. Aad et al. (ATLAS Collaboration), The ATLAS experiment at the CERN Large Hadron Collider. J. Instrum. 3, S08003 (2008). http://cdsweb.cern.ch/record/1129811

3. L. Evans et al., LHC Machine. J. Instrum. 3, S08001 (2008). http:// cdsweb.cern.ch/record/1129806

4. ATLAS/Tile Calorimeter Collaboration, Tile Calorimeter Technical Design Report, CERN/LHCC 96-42, 1996. http://cdsweb. cern.ch/record/331062

5. P. Mermod et al., Effects of ATLAS Tile calorimeter failures on jets and missing transverse energy measurement, ATLAS Note ATL-TILECAL-PUB-2008-011-1， 2008. http://cdsweb.cern.ch/ record/1120460

6. K. Anderson et al., Design of the front-end analog electronics for the ATLAS tile calorimeter. Nucl. Instrum. Methods A 551, 469476 (2005) 
7. S. Berglund et al., The ATLAS Tile Calorimeter digitizer. J. Instrum. 3, P01004 (2008). http://cdsweb.cern.ch/record/1071920

8. A. Valero, on behalf of the ATLAS Tile Calorimeter System, The ATLAS TileCal read-out drivers signal reconstruction, in IEEE Nucl. Sci. Symp. Conference Record, 2009. http://cdsweb. cern.ch/record/1223960

9. J. Poveda et al., Atlas TileCal read-out driver system production and initial performance results. IEEE Trans. Nucl. Sci. 54, 26292636 (2007)

10. P. Adragna et al. (TileCal Collaboration), Testbeam studies of production modules of the ATLAS Tile Calorimeter. Nucl. Instrum. Methods A 606, 362-394 (2009). http://cdsweb.cern.ch/record/ 1161354

11. E. Starchenko et al., Cesium monitoring system for ATLAS Tile Hadron Calorimeter. Nucl. Instrum. Methods A 494, 281-284 (2002). http://cdsweb.cern.ch/record/685349

12. S. Viret, for the LPC ATLAS group, LASER monitoring system for the ATLAS Tile Calorimeter. Nucl. Instrum. Methods A 617, 120-122 (2010). Proceedings of the 11th Pisa Meeting on Advanced Detectors

13. N. Shalanda et al., Radioactive source control and electronics for the ATLAS tile calorimeter cesium calibration system. Nucl. Instrum. Methods A 508, 276-286 (2003)

14. J. Abdallah et al. (TileCal Collaboration), The optical Instrumentation of the ATLAS Tile Calorimeter, ATLAS Note ATLTILECAL-PUB-2008-005，2007. http://cdsweb.cern.ch/record/ 1073936

15. S. Bertolucci et al., Influence of magnetic fields on the response of acrylic scintillators. Nucl. Instrum. Methods A 254, 561-562 (1987)

16. J. Cumalat et al., Effects of magnetic fields on the light yield of scintilators. Nucl. Instrum. Methods A 293, 606-614 (1990)

17. J.-M. Chapuis, M. Nessi, The measurements of magnetic field effects on scintillating tiles, ATLAS Note ATL-TILECAL-94-040, 1994. http://cdsweb.cern.ch/record/683495
18. K. Anderson et al., Calibration of ATLAS Tile Calorimeter at electromagnetic scale, ATLAS Note ATL-TILECAL-PUB-2009-001, 2009. http://cdsweb.cern.ch/record/1139228

19. C. Clement, B. Nordkvist, O. Solovyanov, I. Vivarelli, Time calibration of the ATLAS Hadronic Tile Calorimeter using the laser system, ATLAS Note ATL-TILECAL-PUB-2009-003, 2009. http://cdsweb.cern.ch/record/1143376

20. G. Aad et al. (ATLAS Collaboration), Expected performance of the ATLAS experiment-detector, trigger and physics, CERN Report CERN-OPEN-2008-020, 2009. http://cdsweb.cern.ch/record/ 1125884

21. R. Leitner, V. Shmakova, P. Tas, Time resolution of the ATLAS Tile calorimeter and its performance for a measurement of heavy stable particles, ATLAS Note ATL-TILECAL-PUB-2007002, 2007. http://cdsweb.cern.ch/record/1024672

22. L. de Andrade Filho, J. de Seixas, Combining Hough transform and optimal filtering for efficient cosmic ray detection with a hadronic calorimeter, in XII International Workshop on Advanced Computing Analysis Techniques in Physics Research (Science, 2008)

23. J. Illingworth, A survey of the Hough transform. Comput. Vis. Graph. Image Process. 44, 87-116 (1988)

24. A. Salzburger, The ATLAS Track Extrapolation Package, ATLAS Note ATL-SOFT-PUB-2007-005, 2007. http://cdsweb.cern.ch/ record/1038100

25. Z. Ajaltouni et al., Response of the ATLAS Tile calorimeter prototype to muons. Nucl. Instrum. Methods A 388, 64-78 (1997)

26. L. Fiorini, I. Korolkov, F. Vives, Tile Calibration of TileCal Modules with Cosmic Muons, ATLAS Note ATL-TILECAL-PUB2008-010, 2008. http://cdsweb.cern.ch/record/1109974

27. J. Saraiva, on behalf of the ATLAS Tile Calorimeter System, Commissioning of the ATLAS Tile calorimeter with Single Beam and First collisions, in Proceedings of the 12th Topical Seminar on Innovative Particle and Radiation Detectors, Siena (2010), in Nuclear Physics B (Proceedings Supplement), 2010, to appear. http://cdsweb.cern.ch/record/1281689 